\title{
Pre-clinical development of anginex and design of small-peptide mimetics
}

Citation for published version (APA):

Dings, R. P. M. (2003). Pre-clinical development of anginex and design of small-peptide mimetics.

[Doctoral Thesis, Maastricht University]. Universiteit Maastricht. https://doi.org/10.26481/dis.20030703rd

Document status and date:

Published: 01/01/2003

DOI:

10.26481/dis.20030703rd

Document Version:

Publisher's PDF, also known as Version of record

\section{Please check the document version of this publication:}

- A submitted manuscript is the version of the article upon submission and before peer-review. There can be important differences between the submitted version and the official published version of record.

People interested in the research are advised to contact the author for the final version of the publication, or visit the DOI to the publisher's website.

- The final author version and the galley proof are versions of the publication after peer review.

- The final published version features the final layout of the paper including the volume, issue and page numbers.

Link to publication

\footnotetext{
General rights rights.

- You may freely distribute the URL identifying the publication in the public portal. please follow below link for the End User Agreement:

www.umlib.nl/taverne-license

Take down policy

If you believe that this document breaches copyright please contact us at:

repository@maastrichtuniversity.nl

providing details and we will investigate your claim.
}

Copyright and moral rights for the publications made accessible in the public portal are retained by the authors and/or other copyright owners and it is a condition of accessing publications that users recognise and abide by the legal requirements associated with these

- Users may download and print one copy of any publication from the public portal for the purpose of private study or research.

- You may not further distribute the material or use it for any profit-making activity or commercial gain

If the publication is distributed under the terms of Article $25 \mathrm{fa}$ of the Dutch Copyright Act, indicated by the "Taverne" license above, 


\section{Pre-clinical development of anginex and design of small-peptide mimetics}


() R.P.M. Dings, Minneapolis May 2003 ISBN: $90-9016849-4$

This work was supported by generous support from the National Institutes of Health (NIH, R01 CA-96090; to K.H. Mayo), the US Department of Defense (Army Grant DA/DAMD 17-99-1-9564; to K.H. Mayo), the Dutch Cancer Society (to A.W. Griffioen), and ActiPep Biotechnology, Inc. NMR instrumentation was provided with funds from the National Science Foundation (NSF, BIR-961477) and the University of Minnesota Medical School. 


\title{
Pre-clinical development of anginex and design of small-peptide mimetics
}

\author{
Proefschrift
}

ter verkrijging van de graad van doctor aan de Universiteit Maastricht, op gezag van de Rector Magnificus, Prof. Dr. A.C. Nieuwenhuijzen Kruseman, volgens het besluit van het College van Decanen, in het openbaar te verdedigen op donderdag 3 Juli 2003 om 14:00 uur.

door

Ruud P.M. Dings

geboren te Tegelen op 6 augustus 1977 
Promotor:

Prof. Dr. J. Wagstaff

Co-promotores:

Dr. A.W. Griffioen

Prof. Dr. K.H. Mayo (University of Minnesota)

Beoordelingscommissie:

Prof. Dr. H.A.J. Struijker Boudier (voorzitter)

Prof. Dr. P. Carmeliet (Katholieke Universiteit Leuven)

Prof. Dr. H.F.P. Hillen

Dr. T.L. ten Hagen (Erasmus Universiteit Rotterdam)

Dr. B. Wouters 


\section{Abbreviations}

$\begin{array}{ll}\text { BCE } & \text { bovine microvascular endothelial cells } \\ \text { bFGF } & \text { basic fibroblast growth factor } \\ \text { BPI } & \text { bactericidal-permeability increasing protein } \\ \text { BSA } & \text { bovine serum albumin } \\ \text { CAM } & \text { chick chorioallantoic membrane } \\ \text { CD } & \text { circular dichroism } \\ \text { CP } & \text { cyclic peptide } \\ \text { CRP } & \text { complement regulatory proteins } \\ \text { DMSO } & \text { dimethyl sulfoxide } \\ \text { DNA } & \text { deoxyribonucleic acid } \\ \text { DPC } & \text { dodecylphosphocholine } \\ \text { EC } & \text { endothelial cells } \\ \text { ECM } & \text { extracellular matrix } \\ \text { EGF } & \text { epidermal growth factor } \\ \text { HS } & \text { human serum } \\ \text { HPLC } & \text { high performance liquid chromatography } \\ \text { HUVEC } & \text { human umbilical vein derived endothelial cells } \\ \text { IL-8 } & \text { interleukin- } 8 \\ \text { MMP } & \text { matrix metallo-proteinase } \\ \text { MMPI } & \text { matrix metallo-proteinase inhibitor } \\ \text { NMR } & \text { nuclear magnetic resonance spectroscopy } \\ \text { NOE } & \text { nuclear Overhauser effect } \\ \text { NOESY } & \text { two-dimensional NOE spectroscopy } \\ \text { PE } & \text { phyco-erythrin } \\ \text { PBS } & \text { phosphate buffered saline } \\ \text { PCNA } & \text { proliferating cell nuclear antigen } \\ \text { PDGF } & \text { platelet-derived growth factor } \\ \text { PF4 } & \text { platelet factor } 4 \\ \text { PIGF } & \text { placental growth factor } \\ \text { RF } & \text { radio frequency } \\ \text { RMSD } & \text { root mean square deviation } \\ \text { TIMP } & \text { tissue inhibitors of metallo-proteinase } \\ \text { TNF- } \alpha & \text { tumor necrosis factor- } \alpha \\ \text { TUNEL } & \text { vascular endothelial growth factor } \\ \text { VEGF } & \text { vascular endothelial growth factor receptor } \\ \text { VEGFR } & \\ & \end{array}$




\section{Contents}

Chapter 1 General introduction

Chapter 2 Anti-tumor activity of the novel angiogenesis inhibitor anginex

Chapter 3 The designer anti-angiogenic peptide anginex targets tumor endothelial cells and inhibits tumor growth in animal models

Chapter 4 The designed angiostatic peptide anginex synergistically improves chemotherapy and anti-angiogenesis therapy with angiostatin

Chapter $5 \quad B$-Sheet is the bioactive conformation of the anti-angiogenic anginex peptide

Chapter 6 Design of a partial-peptide mimetic of anginex with anti-angiogenic and anti-cancer activity

Chapter 7 General discussion

Chapter 8 Summary/Samenvatting

Appendix

Acknowledgements / Dankwoord 


\section{Chapter 1}

\section{General introduction}

Partly published in Angiogenesis. 2003; 5(4):1-12. 


\section{Introduction}

Vascularization of organs and tissues proceeds by either of two distinct processes, angiogenesis or vasculogenesis. The first vascular structures in the embryo are formed by vasculogenesis, i.e. the de novo formation of blood vessels from differentiating endothelial cells precursors, or angioblasts. Angiogenesis, the sprouting of capillaries from pre-existing blood vessels, further refines the primitive embryonic vascular system and is the principal process, by which certain organs, such as the brain or the kidney are vascularized. 'In addition, angiogenesis is responsible for repair and remodeling (Table 1). Therefore, it occurs during several developmental stages (such as embryonic growth), but also during several physiological processes in the adult organism, for example during the female menstrual cycle, pregnancy, wound healing and inflammation. Furthermore, angiogenesis plays a major role in the pathogenesis of diseases, such as diabetic retinopathy or retinopathy of prematurity, psoriases, atherosclerosis, obesity, rheumatoid arthritis, peptic ulcers, fractures and cancer. It occurs in embryonic tissues to keep pace with growth, in normal adult tissue to support recurrent cycles of remodeling and reconstruction, and in damaged adult tissues to support repair.

Table 1. The generation of vessels.

\begin{tabular}{|l|l|l|l|}
\hline \multirow{2}{*}{ Vasculogenesis } & \multicolumn{2}{|c|}{ Angiogenesis } \\
\cline { 3 - 4 } & & \multicolumn{1}{|c|}{ Remodeling } & \multicolumn{1}{c|}{ Sprouting } \\
\hline Occurrence & Embryonic development & $\begin{array}{l}\text { Embryonic development } \\
\text { and postnatal }\end{array}$ & $\begin{array}{l}\text { Embryonic development } \\
\text { and postnatal }\end{array}$ \\
\hline Processes & $\begin{array}{l}\text { Progenitor cells } \\
\text { differentiate into } \\
\text { endothelial cells }\end{array}$ & $\begin{array}{l}\text { Pruning and vessel } \\
\text { enlargement }\end{array}$ & $\begin{array}{l}\text { Formation of new blood } \\
\text { vessels from pre-existing } \\
\text { capillaries }\end{array}$ \\
\hline Function & $\begin{array}{l}\text { Establishment vascular } \\
\text { system and structures }\end{array}$ & $\begin{array}{l}\text { Modification of initial } \\
\text { network }\end{array}$ & $\begin{array}{l}\text { Vascularization of } \\
\text { avascular tissue }\end{array}$ \\
\hline
\end{tabular}

\section{Historical}

The evolution of the contemporary theory of angiogenesis within tumor progression has been a gradual process. ${ }^{2}$ The first observations of vascularized tumors date back to the late $19^{\text {th }}$ century. ${ }^{3}$ In the late 1930 s Ide et al. showed that cutting off the blood supply to the tumor led to regression. As a consequence of this study it was proposed, that metastatic tumor cells are dependent upon their ability to maintain a blood supply in order to survive at their new location and that tumor cells elaborate a vessel growth-stimulating substance. ${ }^{4}$ in the early 1970 s, Folkman hypothesized that angiogenesis was an absolute requirement for tumor growth and that interfer- 
ence with the angiogenic process posed an attractive approach for controlling aberrant tumor growth. ${ }^{5,6}$ It was not until the isolation and identification of the first definitive angiogenic factor, basic fibroblast growth factor (bFGF) ${ }^{7}$ and the discovery of the first endogenous angiogenesis inhibitors angiostatin ${ }^{8}$ and endostatin ${ }^{9}$ that the scientific community started to accept Folkman's theory. The research field of angiogenesis has rapidly expanded and there is now a substantial body of evidence that inhibition of angiogenesis can attenuate tumor growth. Now, less then 10 years after the discovery of these anti-angiogenesis molecules, it has become clear that they are not only useful in fighting cancer but also in combating other pathological conditions. ${ }^{10}$ This thesis will focus on inhibiting angiogenesis and the potential of this inhibition in cancer treatment.

\section{Angiogenesis}

Angiogenesis is an integrated process occuring during cancer development. It occurs when tumor tissue is in need of nutrients and/or oxygen. Spontaneous cell death is a common finding in solid tumors. Careful microscopic examination often reveals that the necrotic regions are parallel to tumor blood vessels and separated from them by a 1- to 2-mm zone of viable tumor cells. This size is defined by the diffusion capacity of nutritients, which is about $110 \mu \mathrm{m} .{ }^{11}$ Because of the requirement of the tumor for additional nutrients and oxygen in order to expand, hypoxia is one of the most potent inducers of angiogenesis. ${ }^{12}$ Angiogenesis is considered to arise in the later stages of cancer development but it is clearly occurring before life threatening metastasis develops (Figure 1). Therefore, being able to prevent angiogenesis would provide the possibility of halting metastasis, one of the hallmarks of malignancies. ${ }^{13}$

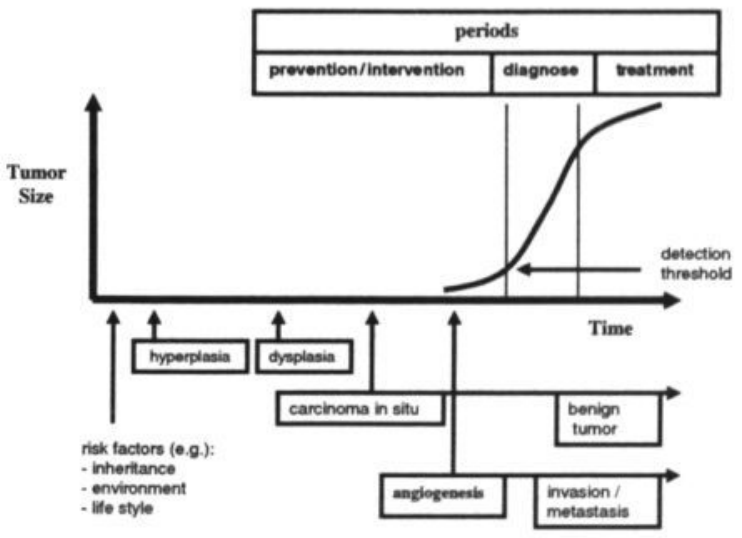

Figure 1. Involvement of tumor angiogenesis in cancer development. Adapted from Kohn et al. ${ }^{14}$ 
Solid tumors and some lymphoma's rely on angiogenic sprouting, to grow beyond a certain size. The induction of angiogenesis by malignant cells has been shown to play a pivotal role in the process of tumor proliferation and metastasis. ${ }^{15,10}$ Angiogenesis is a multi-step sequential process, which can be divided into two major phases. The first, the induction phase, involves dynamic changes in endothelial cell-cell and cell-matrix interaction. In this initiation phase vascular endothelial cells (EC) are activated by angiogenic cytokines, which are released by tumor cells or inflammatory cells.

This process includes degradation of the basement membrane by metallomatrix proteases and the proliferation, migration, and adhesion of endothelial cells. In the second, so called resolution phase, perivascular-supporting cells, pericytes, are recruited and a maturation process results in the assembly of fully functional new blood vessels. ${ }^{16}$ The resulting processes are tightly regulated by cell-cell interactions, cell-extracellular matrix (ECM) interactions and cell-soluble factor interactions. All of these steps involve a plethora of growth factors, receptors, proteases, adhesion molecules, and (ECM) components.

When human tumors arise they are not angiogenesis dependent right away. The switch to an angiogenic phenotype is caused by alterations in the net balance between the positive and negative regulators of angiogenesis. Angiogenesis only occurs when the circulating stimulators are expressed in greater amounts than the inhibitors. Tumor cells, EC, inflammatory cells and the ECM are all capable of releasing angiogenic factors.

It has been postulated that a tumor can acquire other mechanisms, beside angiogenesis, to provide itself with nutrition or drainage, to allow its survival and expansion, locally or at a distant side. Besides angiogenesis, vessel co-option and vascular mimicry have been proposed to be used by the tumor to grow beyond the size of approximately $1-2 \mathrm{~mm}^{3}$. This size is defined by the diffusion capacity of nutrition, which is about $110 \mu \mathrm{m} .{ }^{11}$ However, controversy exists about these latter two processes and it is beyond the scope of this thesis to expand on these proposed mechanisms. For information the reader is referred to review articles with further information about vessel co-option (e.g. Leenders et al. ${ }^{17}$ and Dome et al. ${ }^{18}$ ) and vascular mimicry (e.g. Folberg et al. ${ }^{19}$ and McDonald et al..$\left.^{20}\right)$.

\section{Regulators of Angiogenesis}

As mentioned previously tumor angiogenesis relies on the presence of one or more endogenous pro-angiogenic factors. In Table 2 a summary is listed of the most important regulators of angiogenesis, stimulators as well as inhibitors. This table is not meant to be exhaustive, but rather it is an attempt to exemplify the abundancy of factors involved and consequently the com- 
plexity of the angiogenic process and the possibility of interfering with it.

Table2. A list of angiogenesis regulators.

Angiogenic Regulators

\begin{tabular}{|c|c|}
\hline Stimulators & Inhibitors \\
\hline 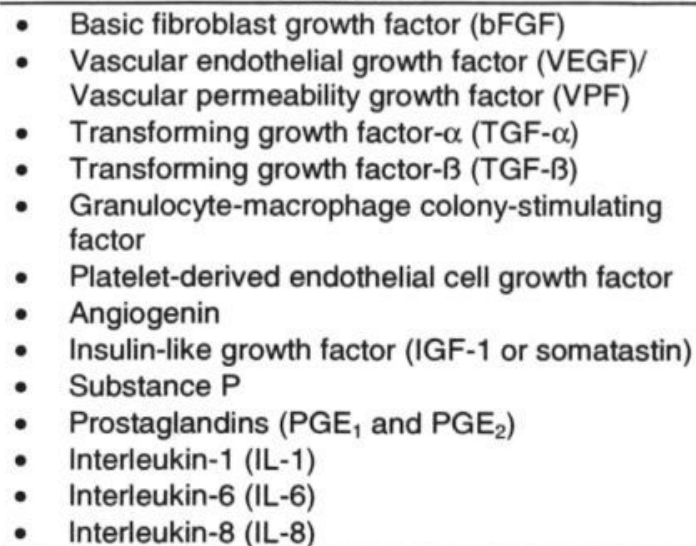 & $\begin{array}{l}\text { ENDOGENOUS } \\
\text { - } \quad \text { Angiostatin } \\
\text { - } \quad \text { Pndostatin } \\
\text { - Interfer factor } 4 \text { (PF4) } \alpha \\
\text { - Interferon- } \gamma \\
\text { - } \quad \text { Thrombospondin-1 } \\
\text { - } \quad \text { BPI } \\
\text { EXOGENOUS } \\
\text { - } \quad \text { Angioinhibin (TNP-470 or AGM-1470) } \\
\text { - } \quad \text { Thalidomide } \\
\text { - } \quad \text { Tissue inhibitors of metallo-proteinases } \\
\text { (TIMPs) }\end{array}$ \\
\hline
\end{tabular}

\section{Stimulators of Angiogenesis}

It is known that each pro-angiogenic factor can promote a different step, or different steps, within the angiogenic process. The most important pro-angiogenic factors, also listed in Table 2 , are bFGF and VEGF. These factors are known to be able to promote specific or all the steps involved in angiogenesis.

\section{Vascular endothelial growth factor}

Vascular endothelial growth factor (VEGF), also referred to as vascular permeability growth factor (VPF), is a potent endothelial-cell-specific mitogen. Next to stimulating angiogenesis, VEGF also causes vascular hyper-permeability and vasodilation. ${ }^{21}$ VEGF expression is known to be increased in endothelial cells under hypoxic conditions. There are at least five polypeptide members of this family $\left(\mathrm{VEGF}_{121}, \mathrm{VEGF}_{145}, \mathrm{VEGF}_{165}, \mathrm{VEGF}_{189}, \mathrm{VEGF}_{206}\right)$ which are generated via an alternative splicing mechanism from an unique gene. The isoforms differ in their ability to bind heparin and in their solubility. The most active form, VEGF ${ }_{165}$ is a $34-46 \mathrm{kDa}$ homodimer, but active heterodimers have also been observed. ${ }^{22}$ The VEGF family currently includes five members in additional to prototypic VEGF, namely, PIGF, VEGF-B, VEGF-C, VEGF-D and Orf virus VEGFs. ${ }^{23}$ The VEGFs mediate angiogenic signals to the vascular endothelium via high-affinity receptor tyrosine kinases (RTK). Three receptors have been indentified. All three are relatively specific for endothelial cells and demonstrate structural and functional similarities to the PDGF receptor family. They all have seven immunoglubulin-like structures in their extracellular domains. 


\section{VEGF-Receptors}

VEGFR-1 (FIt-1) and VEGFR-2 (Flk-1) have seven extracellular immunoglobulin homology domains, but in VEGFR-3 (Flt-4), the fifth immunoglobulin domain is cleaved on receptor processing into disulfide-linked subunits. They all have an intracellular tyrosine kinase signaling domain split by a kinase insert. VEGFR-1 and -2 mediate angiogenesis, whereas VEGFR-3 is restricted to lymphangiogenesis. Neuropilin-1 (NRP-1) binds to specific Cterminal sequences present only in certain VEGFs that bind to VEGFR-1 and/or -2 (Tabel 3). $\alpha_{v} \beta_{3}$ integrin and VE-cadherin have been found in complexes with activated VEGFR-2, and the latter also associates with an activated VEGFR-3 complex.

Table3. The different VEGF receptors, VEGF ligands and their interactions.

\begin{tabular}{c|ccccc}
\hline \multirow{2}{*}{ Receptors } & \multicolumn{5}{c}{ Ligands } \\
\cline { 2 - 6 } & PIGF & VEGF-A & VEGF-B & VEGF-C & VEGF-D \\
\hline sFLT-1 & $\mathrm{X}$ & $\mathrm{X}$ & $\mathrm{X}$ & & \\
VEGFR-1 & $\mathrm{X}$ & $\mathrm{X}$ & $\mathrm{X}$ & & \\
(FIt-1) \\
VEGFR-2 \\
(KDR/FIl-1) & & $\mathrm{X}$ & & $\mathrm{X}$ & $\mathrm{X}$ \\
$\begin{array}{c}\text { VEGFR-3 } \\
\text { (FIt-4) }\end{array}$ & & & & $\mathrm{X}$ & $\mathrm{X}$ \\
$\begin{array}{c}\text { Neuropilin-1 } \\
\text { Neuropilin-2 }\end{array}$ & $\mathrm{X}$ & $\mathrm{X}$ & $\mathrm{X}$ & & \\
\hline
\end{tabular}

VEGF-A binds to both VEGFR-1 and VEGFR-2 and is apparently capable of inducing heterodimers between the two. It has been shown that the second immunoglobulin domain of VEGFR-1 is critical for ligand binding, but the first three immunoglobulin domains are required to reconstitute full affinity.

KDR (VEGFR-2 in humans) is only expressed in endothelial cells, whereas Flk-1 (VEGFR2 in mice) is expressed on both endothelial cells and monocytes. ${ }^{24}$ VEGFR-1 and VEGFR-2 are overexpressed on tumor vasculature, while being present at low density in the surrounding normal tissues. ${ }^{25}$ The upregulation of VEGFR expression is mediated by hypoxia and autocrine stimulation. Specific actions of VEGF on vascular endothelial cells appear to be regulated by both VEGFR-1 and VEGFR-2. VEGFR-1 which has a higher affinity for VEGF than VEGFR-2 is required for endothelial cell morphogenesis, cell migration and vascular permeability. VEGFR-2 is primarily involved in mitogenesis. ${ }^{26.27}$ 


\section{Basic fibroblast growth factor}

Basic fibroblast growth factor (bFGF) is a single chain non-glycosylated cationic polypeptide, with a molecular weight of $18 \mathrm{kDa}$. BFGF has a high affinity for heparin and the bFGFreceptor, and it plays a role in the adhesion of endothelial cells to their stratum. Endothelial cells synthesize bFGF which remains mostly cell associated. However some bFGF becomes deposited in the sub-endothelial extracellular matrix. ${ }^{28}$ Tumor cells can liberate bFGF that is stored in the extracellular matrix by secreting heparin, heparinases and collagenases or by proteolysis mediated by plasminogen activator. BFGF is chemotactic and mitogenic for endothelial cells and they induce bFGF by production of proteolytic enzymes as well as proteoglycans.

\section{Inhibitors of angiogenesis}

Angiogenesis inhibitors have been identified using a myriad of approaches, including (1) isolation and identification of endogenous inhibitors of tumor-promoted angiogenesis ${ }^{9}$, (2) gene therapies ${ }^{29},(3)$ antibody therapies against pro-angiogenic molecules ${ }^{30}$ and (4) their receptors ${ }^{31}$, (5) anti-sense approaches ${ }^{32}$, (6) soluble growth factor receptors as antagonists ${ }^{33}$, (7) interference with growth factor signaling ${ }^{34}$, (8) ribozymes ${ }^{35}$, (9) small molecule inhibitors ${ }^{2}$, and (10) peptide design ${ }^{36}$. The following describes the discovery and development of anti-angiogenic endogenous compounds, with a slant towards their structure-function relationships to aid in the design of better therapeutic agents.

\section{Anti-angiogenic protein fragments}

One approach that has been used to identify molecules with anti-angiogenic potential is to isolate components from the serum or body fluids of animals undergoing extensive angiogenesis, i.e., tumor-bearing or pregnant animals. Many, if not most, of these components were found to be fragments of proteins, which, relative to their parent protein that may or may not be antiangiogenic by themselves, display anti-angiogenic properties when separated from their parent protein. Although endostatin and angiostatin are perhaps the best known examples of such protein fragments, others are also discussed below.

\section{Angiostatin}

The circulating angiogenesis inhibitor angiostatin 8 is a fragment of the non-antiangiogenic plasma protein plasminogen. Angiostatin contains the first four triple-loop disulfide-linked regions of plasminogen, known as kringle domains. ${ }^{8,37}$ This approximately $40 \mathrm{kDa}$ protein was initially isolated from the serum and urine of mice with Lewis lung carcinoma (LLC). Although elastase cleaves plasminogen into an active form of angiostatin in vitro, it is not yet clear which protease(s) is (are) involved in the conversion of plasminogen to angiostatin in vivo. However, it was recently suggested that, depending on the type of tumor, either tumor cells themselves or tumor-associated macrophages express enzymatic activity capable of converting inactive plas- 
minogen into angiostatin. Although plasminogen itself does not bind to the integrin receptor $\alpha_{v} \beta_{3}$, it appears that angiostatin does, suggesting that interaction with $\alpha_{v} \beta_{3}$ is, in part, responsible for the anti-angiogenic properties of angiostatin. ${ }^{38} \mathrm{At}$ least two other possible receptors for angiostatin have been reported: ATP-synthase ${ }^{39}$ and integrin $\alpha_{9} \beta_{1}{ }_{1}{ }^{38}$. Table 4 lists receptors reported for angiostatin and other anti-angiogenic protein fragments and peptides.

Other proteolytic fragments of human plasminogen can also inhibit neovascularization and growth of lung metastases in mice. ${ }^{8}$ For example, kringle 1 (K1) potently inhibits endothelial cell growth, whereas K4 exhibits almost no anti-angiogenic activity in vitro. ${ }^{37}$ Interestingly, K5, which is not present in angiostatin, is structurally related to the other four kringles in plasminogen and also exhibits anti-endothelial activity. ${ }^{40}$

The crystal structure of three kringles from angiostatin folds, as expected for a kringlestructured protein, into anti-parallel $\beta$-sheet and aperiodic loops. ${ }^{41}$ These three kringles are organized in an overall L-shape. Because angiostatin contains a nearly equal number of anionic and cationic residues, the surface of the folded protein is relatively neutral. Nonetheless, the most outstanding electrostatic feature of the protein is a highly electropositive lysine-rich binding site. This is a recurring structural/compositional theme for most anti-angiogenic proteins and peptides identified so far: i.e. anti-parallel $\beta$-sheet structure and preponderance of positively charged and hydrophobic residues.

Table 4. Anti-angiogenic compounds and their reported receptors.

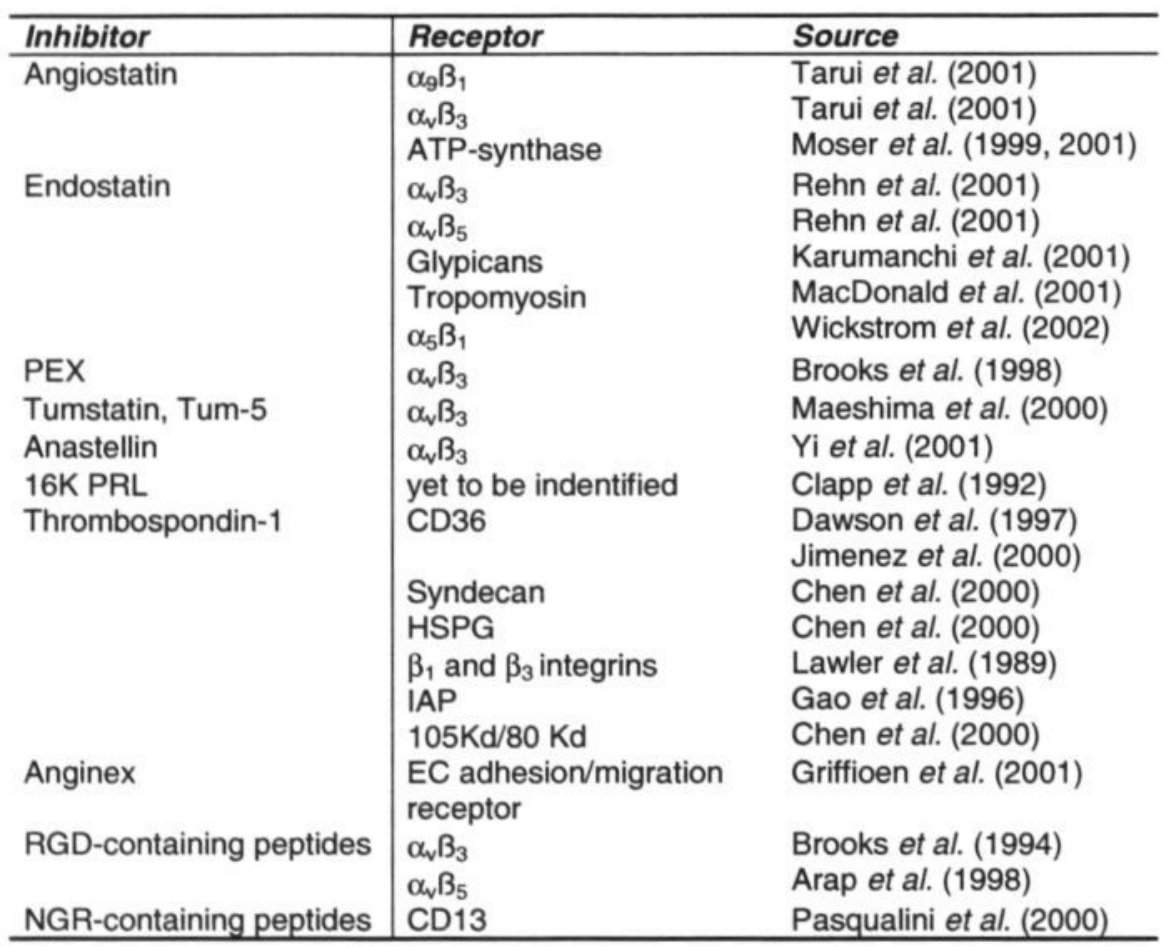




\section{Endostatin}

Endostatin was discovered using a similar approach to that of angiostatin, albeit with hemangioendothelioma as the tumor source. ${ }^{9}$ Analogous to angiostatin, endostatin specifically inhibits proliferation of endothelial cells in vitro and tumor growth in various mouse models in vivo. Furthermore, endostatin has been shown to induce endothelial cell apoptosis in the presence of minimal serum. Endostatin is a $20 \mathrm{kDa}$ C-terminal proteolytic fragment of the NC1 domain of collagen XVIII, which is a member of a family of collagen-like proteins referred to as multiplexins and is localized primarily in the extracellular matrix surrounding blood vessels. The multiplexin family of collagens is distinguished by nearly $60 \%$ sequence identity within the final 184 residues of their $\mathrm{C}$-terminal globular domains, ${ }^{42}$ suggesting that any one of these fragments likewise would be anti-angiogenic. This was recently confirmed by the anti-angiogenic activity of restin, the C-terminal non-collagenous region (NC10) of the $\alpha 1$ chain of human type XV collagen. ${ }^{43}$

The precise mechanism of action for endostatin remains elusive, primarily because endostatin has been found to interact with multiple 'receptors' (Table 4). Endostatin is known to associate with integrin $\alpha_{5} \beta_{1}$, to the anchor protein caveolin-1, and to activate Src via a tyrosyl phosphatase-dependent pathway in human endothelial cells. ${ }^{44}$ These are in addition to earlier reports that endostatin contains a RGD (Arg-Gly-Asp) sequence and binds to integrins $\alpha_{v} \beta_{3}$ and $\alpha_{v} \beta_{5}{ }^{45}$ Furthermore, endostatin, as well as angiostatin, increase cytoplasmic $\mathrm{Ca}^{2+}$ in endothelial cells immediately following exposure. ${ }^{46}$

The crystal structure of endostatin shows the amino acid sequence to be folded into predominantly anti-parallel B-sheet structure, with interspersed extended loops and two short $\alpha$ helices ${ }^{47}$, as illustrated in Figure 2A. The structure of endostatin is homologous to that of Eselectin. ${ }^{48}$ In fact, the entire $\beta$-sheet structure of E-selectin is contained within the endostatin structure. Endostatin is highly positively charged, particularly due to the presence of multiple arginine residues and has a high affinity for heparin, which is required for the full activity of the peptide. ${ }^{49}$

\section{Tumstatin and tum-5}

Recently, the C-terminal globular non collagenous NC1 domain of the $\alpha 3$ chain of human type IV collagen ( $\alpha 3(\mathrm{IV}) \mathrm{NC} 1$ ) called tumstati ${ }^{53}$, was reported to have anti-angiogenic properties. ${ }^{54}$ Aside from its ability to inhibit angiogenesis in vitro and in vivo, tumstatin also inhibits tumor cell proliferation. ${ }^{54,55}$ Using deletion mutagenesis, Maeshima et al. found that the antiangiogenic activity of tumstatin appears to be localized between amino acid residues 54 and 132 , a fragment named tum -5 , whereas its tumor proliferation inhibitory activity resides between amino acid residues 181 and $244 .^{54}$ Maeshima et al. also demonstrated that both tumstatin and tum- 5 bind to $\alpha_{v} \beta_{3}$ (Table 4 ) in an RGD-independent manner and that binding is pivotal for antiangiogenic activity. ${ }^{56}$ In vivo studies demonstrate that at a molar-equivalent concentration, human tum- 5 is at least 10 times more active than human endostatin. ${ }^{57}$ 
A)

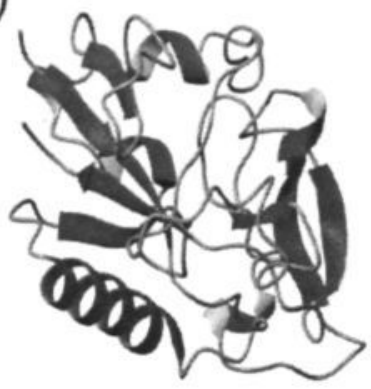

B)

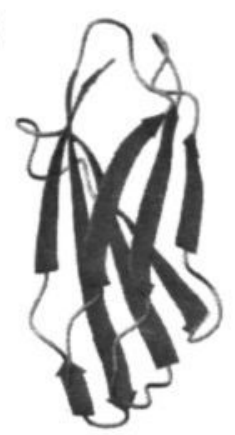

C)

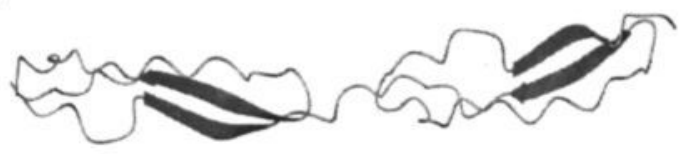

D)

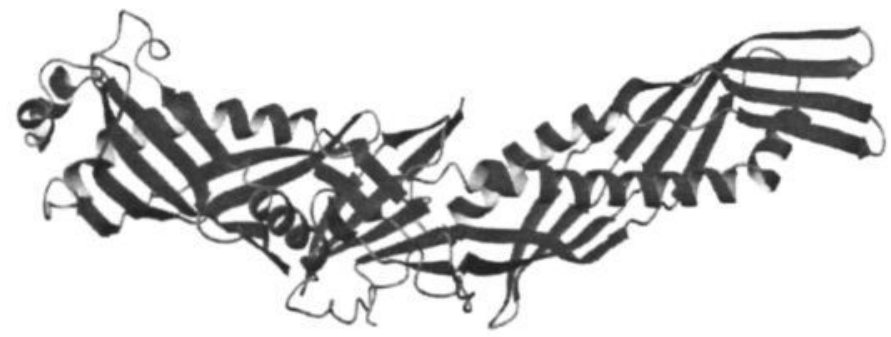

Figure 2. Molecular structures for four anti-angiogenic proteins/peptides.

The molecular structures of endostatin ${ }^{47}$ (panel 2A), fibronectin type III repeat domain ${ }^{50}$ (panel 2B), thrombospondin-1 (TSP-1) $)^{51}$ (panel 2C), and bactericidal permeability increasing (BPI) protein ${ }^{52}$ (panel 2D) are shown.

\section{PEX}

The non-catalytic C-terminal haemopexin-like domain (PEX) of the matrix metallo-proteinase MMP-2 can interact with integrin $\alpha_{v} \beta_{3}$ to block MMP-2 binding and thereby inhibit angiogenesis. MMPs are mosaic proteins containing a $\mathrm{N}$-terminal pro-domain, a catalytic $\mathrm{Zn}^{2+}$ protease domain and the C-terminal PEX haemopexin-like domain. PEX2 appears to be a naturally occurring breakdown product of MMP-2. This protein fragment can inhibit cell-associated collagenolytic activity with preferential substrate specificity towards type IV collagen, which is thought to play an important role in endothelial cell proliferation and behaviour during the angio- 
genic process because it is a major macromolecular constituent of basement membranes. ${ }^{16}$ Consequently, PEX inhibits angiogenesis and, in this way, controls normal angiogenesis and neovascularization. Since MMP-2 does not contain the RGD sequence, PEX binding to $\alpha_{v} \beta_{3}$ (Table 4) is RGD-independent. Therefore, exogenously administered PEX may be a therapeutic inhibitor of diseases associated with angiogenesis ${ }^{58}$ and may be less toxic than treatment with MMP enzyme inhibitors themselves. ${ }^{59}$

The structure of the PEX domain in MMP9 is a non-covalent asymmetric homodimer, consisting of multiple $\beta$-sheets. The overall structure of PEX9 adopts a four-bladed B-propeller, and blade IV of PEX9 mediates the non-covalent and predominantly hydrophobic dimerisation contact. $^{60}$

\section{Anastellin}

Anastellin is another anti-angiogenic peptide ( 76 amino acid residues), derived from the first type III repeat of fibronectin ${ }^{61}$, which contains a site important in fibronectin self-assembly into fibrils. ${ }^{62}$ Anastellin may disrupt intermolecular interactions that maintain fibronectin in its soluble form ${ }^{62}$, and this disruption may induce the molecule to undergo assembly into fibrils. ${ }^{61}$ Alternatively, anastellin may change the conformation of fibronectin in such a way that cryptic fibronectinfibronectin binding sites that are capable of driving fibril assembly are exposed. Systemic treatment of tumor-bearing mice with anastellin suppresses the growth of subcutaneous tumors and inhibits tumor angiogenesis, as well as metastasis ${ }^{63}$.

This first type III repeat in fibronectin has a similar function as the NC1 domain from collagen IV. This suggests a common mechanism for anti-angiogenic substances derived from extracellular matrix and blood proteins ${ }^{63}$, especially considering most of these anti-angiogenic substances bind to adhesion molecules via the RGD-sequence. For instance, angiostatin binds to vitronectin, endostatin binds to fibulins, and nidogen-2 and anastellin bind to fibronectin and fibrinogen in serum..$^{63}$ Moreover, all bind to $\alpha_{v} \beta_{3}$ (Table 4), which is expressed at high levels in angiogenic endothelial cells. ${ }^{64}$

As shown in Figure 2B, the fibronectin type III domain consists of seven $\beta$-strands folded into two anti-parallel $\beta$-sheets having an immunoglobulin-like fold similar to that found in homologous modules in the cytokine receptor superfamily. ${ }^{50,65}$ Moreover, the surface of this molecule is highly positively charged.

\section{Others}

Prolactin, growth hormone and placental lactogen are members of a family of polypeptide hormones that share structural similarities and biological activities. They modulate angiogenesis along with two non-classical members of the family, proliferin and proliferin related protein. Opposing actions have been described between these two similar, yet independent, molecules (proliferin and proliferin related protein), which can stimulate and inhibit angiogenesis, respec- 
tively. The potential to exert opposing effects on angiogenesis can also reside within the same molecule as the parent protein promotes angiogenesis (i.e. prolactin, growth hormone and placental lactogen), but after proteolytic processing, the resulting peptide fragment acquires antiangiogenic properties (i.e. $16 \mathrm{kDa}$ prolactin, $16 \mathrm{kDa}$ growth hormone and $16 \mathrm{kDa}$ placental lactogen). ${ }^{66-70}$

Endothelial cells are known to produce various forms of laminin, but the structural characteristics and biological features are incompletely known. ${ }^{71}$ Whereas laminin itself, as well as peptides derived from it, are pro-angiogenic ${ }^{72,73}$, vasostatin (residues 1-180 of the $\mathrm{NH}_{2}$ domain of calreticulin) can bind to laminin, thus promoting anti-angiogenic activity. Calreticulin by itself is also anti-angiogenic. ${ }^{71}$ Domain IVa of the laminin $\alpha 5$ chain is likely to be responsible for the angiogenic response of laminin. This domain promotes cell adhesion and binds to $\beta_{1}$ and $\alpha_{v} \beta_{3}$ integrins via the RGD sequence.$^{74}$ In addition, the laminin receptor $67 L R$ plays an important role in metastasis, tumor invasiveness and tumor angiogenesis because it is upregulated during these conditions. Interestingly, the RDGSYGIV peptide derived from epidermal growth hormone (residues 33-42), which inhibits endothelial cell motility, is an antagonist for 67LR. ${ }^{75}$

Lastly, the metal-binding glycoprotein osteonectin (also called BM-40 or SPARC) has a protruding $\mathrm{N}$-terminal $\mathrm{B}$-hairpin with striking similarities to epidermal growth factor (EGF). ${ }^{76} \mathrm{Al}$ though the molecular structure of osteonectin consists mostly of helices, this ß-hairpin structure is believed to be the site that promotes anti-angiogenic activity. ${ }^{76}$

\section{Anginex}

As discussed above, most anti-angiogenic agents are endogenous molecules or derived from endogenous molecules, primarily proteins. On the other hand, our laboratories reported the potent anti-angiogenic activity of a designed peptide 33mer called anginex or $\beta$ pep-25. ${ }^{36,77}$ Anginex specifically targets angiogenically-activated endothelial cells, inhibiting EC adhesion to and migration on the extracellular matrix and thereby inducing apoptosis. Although no specific receptor for anginex has yet been identified, it appears that one or more adhesion/migration receptor(s), upregulated in angiogenically-activated endothelial cells, is (are) involved..$^{36}$

NMR structural studies show that anginex folds amphipathically into a three-stranded antiparallel $\beta$-sheet motif. ${ }^{78}$ The hydrophilic face of the folded peptide carries a high net positive charge (+5), mostly from lysine residues. This fold and composition is similar to that found in a number of other $\beta$-sheet-structured, anti-angiogenic cytokines, such as TNF- $\alpha^{79}$, lymphotoxin (LT or TNF- $\beta)^{80}$, transforming growth factor- $\beta$ (TGF- $\left.\beta\right)^{81}$, and bactericidal/permeability increasing protein $(\mathrm{BPI})^{52,82}$. The structure of $\mathrm{BPI}$ is shown in Figure $2 \mathrm{D}$ to illustrate the extent of its $\beta$-sheet fold. Although anginex contains a short $\beta$-strand sequence identical to one from $\mathrm{BPI}$, that same synthetic sequence from BPI demonstrates no anti-angiogenic activity, indicating that it is the unique anginex sequence and fold that impart anti-angiogenic function. 


\section{Anti-angiogenesis therapy}

Solid tumors are composed of two interdependent compartments: the parenchyma (tumor cells) and the stroma (supporting cells and connective tissue). The latter provides the vascularity and is the main target of anti-angiogenic therapy. The parenchyma can still be targeted with conventional therapies. Targeting new anti-cancer therapies to vascular endothelial cells has a number of advantages over treatments that directly target tumor cells: (i) endothelial cells are in direct contact with the blood and are therefore more accessible to plasma soluble agents, (ii) unlike tumor cells, endothelial cells are genetically stable cells that are not easily mutated into drug resistance variants, (iii) angiogenesis-inducing pathways may be similar in different tumor types, promoting the broad applicability of anti-angiogenic intervention, (iv) elimination of a single endothelial cell can eradicate the growth of over 100 tumor cells.

\section{Possible drawbacks of angiogenesis inhibitors}

As described above endogenous angiogenic inhibitors were necessary to prove that aberrant tumor growth could be halted. Beside their functionality in vitro these endogenous molecules face some difficulties in applicability in vivo.

\section{Contextual Angiogenesis Modulators}

The complexity of tumor-induced angiogenesis rests in part in the micro-environment of a given tumor, which further complicates development of a highly effective anti-angiogenic agent. For example, hypoxia and ischemia trigger the production of angiogenic survival factors, such as VEGF, but also of endothelial apoptosis-inducing factors like TNF- $\alpha{ }^{83.84}$ The level of production of these factors is important because some cytokines, such as TNF- $\alpha$ and TNF- $\beta$, can function both as stimulators and inhibitors of angiogenesis depending upon their concentration. Another example is thrombospondin-2 (TSP-2), which displays anti-angiogenic activity ${ }^{85,86}$, via secretion from fibroblasts, and possibly other mesenchymal cells, as a paracrine mediator of EC prolifera$\operatorname{tion}^{87}$. Such agents are therefore contextual inhibitors of angiogenesis that depend on their presence within a particular physiological environment.

Thus, depending on the interaction within the particular habitat, a factor will either be produced or not and in a certain amount by paracrine or autocrine matter and act accordingly.

\section{Immunity}

One of the advantages of treatment with endogenous inhibitors of angiogenesis over that of exogenous inhibitors is the absence of possible immunity against the particular compound. Recently, however, the presence of anti-endostatin antibodies in patients with multifocal glioblastoma has been reported. Interestingly, these auto-immune antibodies were observed in patients with aggressive tumors, but not in patients with less aggressive tumors. ${ }^{88}$ Further analysis will be 
necessary to determine their functional role in tumor angiogenesis. However, it is possible that these antibodies reduce effectiveness of endostatin therapy and therefore provide an explanation as to how tumors can escape the effects of angiogenic molecules.

\section{Specificity}

In the field of cancer research, a prerequisite for the use of angiostatic compounds is the specificity for tumor endothelial cells. In laboratory research this is being translated into specificity for activated (recently stimulated) endothelial cells. A number of receptors of endogenous inhibitors that are overexpressed on activated endothelial cells (to mimic tumor EC) are mentioned prior.

Endogenous angiogenesis inhibitors seem to target multiple receptors, as described above (Table 4), and can be involved in different physiological processes, which can entail problems as exemplified by the following. Small molecule inhibitor SU5416, inhibits VEGF mediated Flk-1/KDR receptor signaling. It has been shown that SU5416 inhibits tumor vascularization and growth in xenograft models..$^{34}$ In human trials, when combined with chemotherapy (cisplatin and gemcitabine) it has shown severe toxicity. Despite careful selection of the patients, this combinatorial treatment showed an extraordinarily high incidence of vascular and venous events. ${ }^{89}$ It has been suggested that there might be a crucial link between the angiogenic process and the coagulation system, potentially giving rise to the toxicity. This hypothesis has been questioned on account of lack of toxicity when SU5416 is combined with other chemotherapeutic agents, including fluorouracil, irinotecan, carboplatin, and paclitaxel. ${ }^{90}$ Therefore future development of anti-angiogenic compounds should be highly specific for upregulated receptors on activated tumor endothelial cells. This should circumvent interference in physiological angiogenic processes and possible cross-talk with for example the coagulation system or bone and cartilage remodeling.

\section{Aim of presented thesis: design and development of novel angiogen- esis inhibitors for cancer treatment}

Although preclinical testing of anti-angiogenic agents shows promise, the need for more and better angiogenesis inhibitors is driven by the absence of any major clinical breakthroughs (e.g. SU5416, BB2516, AG3340, Bay 12-9566, IM-862). ${ }^{91}$ These therapeutic molecules inhibit neovascularization at various steps of blood vessel formation. For example, several matrix metalloproteinase inhibitors block the degradation of the endothelial basement membrane, thus preventing capillary sprout formation. Development of antagonistic reagents by blocking angiogenic ligand/receptors-mediated signaling pathways has become one of the most attractive approaches in anti-angiogenic therapy. These strategies are mainly aimed at inhibition of VEGF, 
one of the specific key angiogenic factors secreted by tumors. However, these single angiogenic factor antagonists may encounter major problems in development of drug resistance, as tumor cells may, most likely, switch their angiogenic stimulators. As cancer masses consist of heterogeneous populations of tumor cells, the anti-VEGF reagents might stress tumor cells to select for colonies producing other angiogenic factors such as fibroblast growth factor-2 (FGF2). Thus VEGF antagonists may not be effective or only temporary in the treatment of all cancers. This is shown by the observation that long-term blocking of VEGF activity resulted in a second wave of VEGF-independent angiogenesis..$^{92}$ In contrast, general angiogenesis inhibitors that block common pathways of tumor angiogenesis can bypass drug resistance and be therapeutically effective against all cancer types. Angiostatin and endostatin are two such specific endothelial cell angiogenesis inhibitors, which have entered clinical trials. According to animal studies, relatively large dosages $(20-100 \mathrm{mg} / \mathrm{kg}$ ) of these angiogenesis inhibitors have to be delivered in order to reach maximal effects. When these amounts are translated into human trials, it seems unattractive to deliver huge amount of biologically active recombinant proteins for longterm treatments of large numbers of cancer patients. Besides the requirement for high dosages, possible difficulties manufacturing active molecules and high costs, there is a chance of introducing transmission of micro-organism toxins from the recombinant proteins. Thus, it seems impractical to apply protein angiogenesis inhibitors to a large number of patients. ${ }^{93}$ Therefore, development of new endothelial cell angiogenesis inhibitors, especially synthetic small peptides, is a potentially important approach for improving or even replacing current anti-angiogenic therapies.

\section{Outline of the thesis}

A library of peptides was designed using a combinatorial approach employing basic folding principles and incorporation of amino acid sequences of $B$-sheet containing angiogenesis inhibitors. ${ }^{94.95}$ By the use of in vitro anti-angiogenesis assays one of the peptides in the library (Bpep-25) anginex was identified as being the most effective in proliferation and migration inhibition and induction of apoptosis. ${ }^{36} \mathrm{Here}$, it is shown for the first time that anginex also posses anti-tumor activity in vivo. The aim of the chapters 2 and 3 was to test and to optimize the in vivo efficacy of anginex as an anti-cancer agent in both synergic as xenograft mouse models. Chapter 4 describes the combinatorial effect anginex had with angiostatin, a well-know angiogenesis inhibitor, as well with conventional chemotherapeutic agent carboplatin. Chapter 5 discusses the importance of the $B$-sheet structure of anginex for its anti-angiogenic capacity, followed by chapter 6 , which describes the structural activity relationships within anginex resulting in the design and development of a new generation of peptide-mimetics. Chapter 7 is a general discussion and finally a summary is given in chapter 8 . 


\section{Chapter 2}

\section{Anti-tumor activity of the novel angiogenesis inhibitor anginex}

Ruud P. M. Dings, Daisy W. J. van der Schaft, Balazs Hargittai, Judy R. Haseman, Arjan W. Griffioen, and Kevin H. Mayo

Cancer Lett. 2003; 194(1): 55-66. 


\section{Introduction}

Angiogenesis or neovascularization is the process through which new blood vessels develop from pre-existing vasculature. ${ }^{96}$ The growth of solid tumors is dependent on angiogenesis, as tumors generally can not grow beyond the size of 1-2 mm in diameter without formation of new blood vessels to supply nutrition and oxygen and remove waste products. Most tumors release angiogenesis-regulating factors, and neovasculatization occurs only when there are more positive than negative regulators of angiogenesis. ${ }^{97,98}$

A highly vascularized tumor is correlated with a poor clinical prognosis, not only because of the potential for uncontrolled tumor growth, but also because of the increased access of the tumor to capillaries, which contributes to increased metastatic potential. ${ }^{99.100}$ Consequently, methods that inhibit angiogenic sprouting provide a unique opportunity to arrest tumor growth and prevent metastasis formation. Many endogenous and exogenous anti-angiogenic agents have been identified, such as angiostatin ${ }^{8}$, endostatin ${ }^{9}$, thrombospondin ${ }^{101}$, TNP- $470^{102}$, platelet factor- $4^{103}$, thalidomide ${ }^{104}$, squalamine ${ }^{105}$, bactericidal/ permeability-increasing protein ${ }^{82}$ and carboxyaminoimidazole ${ }^{106}$.

Although preclinical testing of anti-angiogenic agents shows promise, the need for more and better angiogenesis inhibitors is driven by the absence of any major clinical breakthroughs (e.g. SU5416, BB2516, AG3340, Bay 12-9566, IM-862). ${ }^{91}$ The most successful angiostatic agents have been those that directly act by inhibiting endothelial cell proliferation. Anginex, a designed B-sheet-forming cytokine-like 33-mer peptide, which potently inhibits multiple steps in the angiogenesis process, is a member of this class of angiogenesis inhibitors. A family of homologous Bpep peptides was designed by using basic folding principles and incorporating short sequences from the $\beta$-sheets domains of $\alpha$-chemokine IL- 8 and bactericidal/permeability increasing (BPI) protein. ${ }^{36}$ The angiostatic activity of anginex is based primarily on apoptosis-induction via prevention of adhesion on and migration through the extracellular matrix of angiogenically-activated endothelial cells. ${ }^{36}$

The aim of the present study was to test and to optimize the in vivo efficacy of anginex as an anti-cancer agent in mouse models. Various treatment regiments, i.e., dose response, mode and frequency of administration, were investigated. For other angiogenesis inhibitors, the literature reports that continuous, systemic administration of, for example endostatin, results in more effective tumor growth suppression at significantly reduced doses compared with bolus administration. ${ }^{107}$ Continuous systemic administration of anginex in a $10 \mathrm{mg} / \mathrm{kg} /$ day dose provided optimal efficacy. Efficacy was significantly improved by conjugating anginex to human serum albumin to improve its bioavailability. These results demonstrate that anginex is a promising pharmacological anti-cancer agent that should be developed for clinical testing. 


\section{Introduction}

Angiogenesis or neovascularization is the process through which new blood vessels develop from pre-existing vasculature. ${ }^{96}$ The growth of solid tumors is dependent on angiogenesis, as tumors generally can not grow beyond the size of $1-2 \mathrm{~mm}$ in diameter without formation of new blood vessels to supply nutrition and oxygen and remove waste products. Most tumors release angiogenesis-regulating factors, and neovasculatization occurs only when there are more positive than negative regulators of angiogenesis..$^{97,98}$

A highly vascularized tumor is correlated with a poor clinical prognosis, not only because of the potential for uncontrolled tumor growth, but also because of the increased access of the tumor to capillaries, which contributes to increased metastatic potential. ${ }^{99.100}$ Consequently, methods that inhibit angiogenic sprouting provide a unique opportunity to arrest tumor growth and prevent metastasis formation. Many endogenous and exogenous anti-angiogenic agents have been identified, such as angiostatin ${ }^{8}$, endostatin ${ }^{9}$, thrombospondin ${ }^{101}$, TNP- $470^{102}$, platelet factor- $4^{103}$, thalidomide ${ }^{104}$, squalamine ${ }^{105}$, bactericidal/ permeability-increasing protein ${ }^{82}$ and carboxyaminoimidazole ${ }^{106}$.

Although preclinical testing of anti-angiogenic agents shows promise, the need for more and better angiogenesis inhibitors is driven by the absence of any major clinical breakthroughs (e.g. SU5416, BB2516, AG3340, Bay 12-9566, IM-862). ${ }^{91}$ The most successful angiostatic agents have been those that directly act by inhibiting endothelial cell proliferation. Anginex, a designed B-sheet-forming cytokine-like 33-mer peptide, which potently inhibits multiple steps in the angiogenesis process, is a member of this class of angiogenesis inhibitors. A family of homologous Bpep peptides was designed by using basic folding principles and incorporating short sequences from the $\beta$-sheets domains of $\alpha$-chemokine IL- 8 and bactericidal/permeability increasing (BPI) protein. ${ }^{36}$ The angiostatic activity of anginex is based primarily on apoptosis-induction via prevention of adhesion on and migration through the extracellular matrix of angiogenically-activated endothelial cells. ${ }^{36}$

The aim of the present study was to test and to optimize the in vivo efficacy of anginex as an anti-cancer agent in mouse models. Various treatment regiments, i.e., dose response, mode and frequency of administration, were investigated. For other angiogenesis inhibitors, the literature reports that continuous, systemic administration of, for example endostatin, results in more effective tumor growth suppression at significantly reduced doses compared with bolus administration. ${ }^{107}$ Continuous systemic administration of anginex in a $10 \mathrm{mg} / \mathrm{kg} /$ day dose provided optimal efficacy. Efficacy was significantly improved by conjugating anginex to human serum albumin to improve its bioavailability. These results demonstrate that anginex is a promising pharmacological anti-cancer agent that should be developed for clinical testing. 


\section{Materials and Methods}

\section{Cell Culture}

The human epithelial ovarian carcinoma cell line, MA148, was kindly provided by Prof. Dr. Ramakrishnan. MA148 cells were cultured at $37^{\circ} \mathrm{C}$ in RPMI 1640 medium (Life Technologies, Grand Island, NY) supplemented with $10 \%$ fetal bovine serum (Cellgro, Mediatech, Washington, DC) and $1 \%$ penicillin/streptomycin (Cellgro, Mediatech). Cultures were grown in the presence of $5 \% \mathrm{CO}_{2}$ and split $1: 3$ every 3 days. The human SK-OV-3 carcinoma cell line was kindly provided by Dr. Rebecca Bagley (Genzyme).

\section{Proliferation assay}

Human umbilical vein derived $\mathrm{EC}$ were harvested from normal human umbilical cords by perfusion as described earlier. ${ }^{36}$ HUVEC were seeded in a 96 wells culture plate coated with 1 $\mathrm{mg} / \mathrm{ml}$ fibronectin $\left(2\right.$ hours at $20^{\circ} \mathrm{C}$ ) at a concentration of 3,000 cells per well in a volume of 50 $\mu \mathrm{l}$. MA148 cells were seeded in a non-coated 96 wells culture plate at a concentration of 5,000 cells per well in a volume of $50 \mu \mathrm{l}$. Both cell types were allowed to adhere for 3 hours at $37^{\circ} \mathrm{C}$ at $5 \% \mathrm{CO}_{2}$ and subsequently $50 \mu \mathrm{l}$ of culture medium with $20 \mathrm{ng} / \mathrm{ml}$ basic Fibroblast Growth Factor (bFGF), with or without anginex or conjugated anginex was added. The cells were cultured for 72 hours. Two different proliferation assays were used to quantify the effect of the treatments, $\left[{ }^{3} \mathrm{H}\right]$-thymidine incorporation and the cell counting kit (CCK) was used. $0.5 \mu \mathrm{Ci}$ [methyl- ${ }^{3} \mathrm{H}$ ]thymidine(Amersham Life Science)/well was used to pulse the culture, during the last 6 hours of the assay and activity was measured using liquid scintillation. $10 \mu \mathrm{l}$ of CCK-8 solution (Dojindo, Gaithersburg, Maryland) was added to the culture during the last 2 hours of the assay. This was then quantified using a microplate reader, and the absorbance was measured at $450 \mathrm{~nm}$ with the reference wavelength at $650 \mathrm{~nm}$. All measurements were done in triplicate, and the experiments were done at least three times.

\section{In vitro angiogenesis}

Sprouting and tube formation of EC were studied using cytodex-3 beads overgrown with $E C$ in a 3-dimensional gel. Bovine capillary EC (BCE) were mixed with gelatin coated cytodex3 microcarrier beads (Sigma, The Netherlands) at a concentration of 25 cells per bead and cultured for 72 hours in a tissue culture plate in RPMI-1640, supplemented with $20 \% \mathrm{HS}, 2 \mathrm{mM}$ L-glutamine, $50 \mathrm{ng} / \mathrm{ml}$ streptomycin, and $50 \mathrm{U} / \mathrm{ml}$ penicillin. The beads were spun down and resuspended, in a concentration of 25 beads per $100 \mu$, in 8 volumes of vitrogen- 100 (Collagen Corporation, Fermont, CA, USA), 1 volume 10x concentrated $\alpha$-MEM (Life Technologies, Breda, The Netherlands), 1 volume $11.76 \mathrm{mg} / \mathrm{ml}$ sodium bicarbonate and $20 \mathrm{ng} / \mathrm{ml} \mathrm{bFGF.} 100 \mu \mathrm{l}$ of this mixture was suspended to each well of a 96 wells culture plate, after which gelation was allowed to take place at $37^{\circ} \mathrm{C}$. After gelation medium was applied on top of the gel containing $20 \mathrm{ng} / \mathrm{ml}$ 
bFGF, with or without anginex at concentrations as indicated. After 24 hours photographs were made. For quantification these images were analyzed using $\mathrm{NIH}$ image computer software. Statistical analysis was done using the Mann-Whitney $\mathrm{U}$ test.

\section{Animals}

In all studies, female athymic nude mice (nu/nu, 5-6 weeks old) were used. These mice were purchased from the National Cancer Institute and allowed to acclimatize to local conditions for at least one week. Animals were given water and standard chow ad libitum, and were kept on a 12-hour light/dark cycle. All experiments were approved by the University of Minnesota Research Animal Resources ethical committee.

\section{Xenograft mouse models}

MA148 cells, growing in exponential phase, were harvested by trypsinization, washed with Hanks' balanced salt solution (Cellgro, Mediatech) and resuspended at $2 \times 10^{7} \mathrm{cells} / \mathrm{ml}$ in serum-free RPMI- 1640 medium. $100 \mu$ l of the suspension was injected subcutaneously into the right flank of the mouse. For mice with minimal residual disease, treatment was initiated at the time of inoculation with MA148 cells. As a therapeutic intervention variant, tumors were allowed to grow to an average size of $50 \mathrm{~mm}^{3}$ (day 17 post-inoculation) before treatment was initiated.

Another therapeutic intervention model was performed with a more aggressive carcinoma, human SK-OV-3, where tumors were allowed to establish to an average size of approximately $75 \mathrm{~mm}^{3}$ before treatment was initiated. In either case, animals were randomized prior to the initiation of treatment.

\section{Treatment variations}

Three variations in treatment administration were employed: loco-regional injections, alginate beads and osmotic mini-pumps. Loco-regional administration was performed by injecting agents subcutaneously in the right flank within $1 \mathrm{~cm}$ of the tumor, rather than directly into the tumor mass. Single and double daily injections were performed. Double daily injections were administered at 12-hour intervals. Alginate beads and osmotic mini-pumps were both used in order to compare two different continuous and systemic modes of treatment. Alginate beads, a simple, mild, aqueous-based gel formation of sodium alginate in the presence of divalent cations such as $\mathrm{Ca}^{2+}$, were made using alginic acid (Sigma Chemical Co.), with some modifications from the method previously described. ${ }^{108}$ Briefly, the peptide anginex was dissolved in $2 \%$ $\mathrm{w} / \mathrm{v}$ sodium alginate solution, which was then extruded through a $30-\mathrm{G}$ needle into a $0.1 \mathrm{M}$ calcium chloride solution while stirring. Alginate beads were stored overnight at $4{ }^{\circ} \mathrm{C}$ and subsequently washed three times in sterile distilled $\mathrm{H}_{2} \mathrm{O}$. Each week, these beads were made fresh and placed subcutaneously in the left flank of the animals, achieving a dose of $10 \mathrm{mg} / \mathrm{kg} /$ day. In studies using osmotic mini-pumps (Durect, Cupertino, CA), mini-pumps were implanted subcu- 
taneously in the left flank of mice. Concentrated solutions of anginex were formulated such that the 28-day treatment period would be covered by implantation of a single pump.

For each route of administration, control groups of animals were given either PBS, PBS containing human serum albumin, or PBS containing a negative control peptide Bpep-28 that is $91 \%$ sequentially homologous and $67 \%$ identical to anginex..$^{36}$ Tumor growth curves were found to be virtually identical in any of these control cases.

\section{Tumor volume measurements}

Tumor volume was determined by measuring the diameters of tumors using calipers (Scienceware, Pequannock, $\mathrm{NJ})$ and calculated by the equation for the volume of a spheroid: $\left(\mathrm{a}^{2}\right.$ $\mathrm{xb} x \pi) / 6$, where ' $a$ ' is the width and ' $b$ ' is the length of the tumor. Measurements were performed two or three times per week. At the conclusion of an experiment, tumor weights were also taken following excision of the tumors from euthanized animals. Statistical analysis was performed using the Student's t-test.

\section{Anginex-HSA conjugate}

Given that small peptides like anginex are subject to rapid clearance via the kidney, a study was performed in which anginex was coupled to a larger carrier protein, human serum albumin (HSA). To produce this conjugate, five milligrams of HSA (Aldrich Chemicals, Milwaukee, WI) was dissolved in $1 \mathrm{ml}$ of $50 \mathrm{mM} \mathrm{NaPO}_{4}$ buffer, $\mathrm{pH} 7.5$, containing $1 \mathrm{mM}$ EDTA. Freshly dissolved S-acetylthioacetic acid (SATA, $65 \mathrm{mM}$ in dimethyl sulfoxide) was then added and stirred at $25^{\circ} \mathrm{C}$ for 1 hour. ${ }^{109}$ The resulting derivatized protein was separated from smaller molecules by gel filtration using Sephacryl S-200HR (Amersham Pharmacia Biotechnology). To the collected and combined fractions, a $0.5 \mathrm{M}$ aqueous solution of hydroxylamine hydrochloride (in $\mathrm{PO}_{4}$ buffer containing $25 \mathrm{mM}$ EDTA; $\mathrm{pH} 7.5$ ) was added and stirred at $25^{\circ} \mathrm{C}$ for 1.5 hours in order to remove the acetyl-protecting group. ${ }^{110}$ The amount of free sulfhydryl was measured using dinitrothiobenzoic acid (Ellman's reagent). ${ }^{11}$ There were, on average, five free sulfhydryls per HSA molecule.

Following gel purification, the derivatized protein was treated with succinimidyl 4-( $\mathrm{N}-$ maleimidomethyl)cyclohexane-1-carboxylate ( $47 \mathrm{mM}$ in dioxane) that acted as the cross-linker. The reagent was added in nine aliquots of $4 \mu \mathrm{l} / \mathrm{ml}$ at 5 -minutes intervals under gentle agitation, and gently shaken overnight at $32^{\circ} \mathrm{C}$. The resulting protein was purified by gel filtration, lyophilized and redissolved in $\mathrm{H}_{2} \mathrm{O}(1 \mathrm{ml}) .1 \mathrm{mg}$ anginex, dissolved in $\mathrm{H}_{2} \mathrm{O}(0.25 \mathrm{ml})$, was then added to the protein solution. The resulting cloudy solution was stirred at $25^{\circ} \mathrm{C}$ overnight, centrifuged to remove any precipitate, purified using gel filtration, and finally dialyzed and lyophilized to give the desired conjugate. The protein was characterized by HPLC and Mass Spectrometry. From Mass Spectrometry, it was estimated that there was an average of 5 anginex molecules per HSA molecule. An EC proliferation assay (incorporation of $\left[{ }^{3} \mathrm{H}\right]$ thymidine) showed no enhanced activity of 
conjugated anginex compared to its molecular equivalent of free anginex.

\section{Immunohistochemistry}

Immunohistochemistry was used to assess microvessel density and the extent of total cell death. Approximately, tumors of the same size were selected for processing. Tumor tissue was embedded in tissue freezing medium (Miles Inc, Elkart, IN) and shock frozen in liquid nitrogen. $10 \mu \mathrm{m}$ thick sections of tissue were prepared for immunohistochemical analysis. For this, tissue sections were brought to room temperature, air dried overnight, and then fixed in acetone for 10 minutes. Slides were allowed to air dry for at least $\mathbf{3 0}$ minutes and were washed three times for 5 minutes each in phosphate-buffered saline (PBS, pH 7.4). Samples were then blocked with PBS containing $0.1 \%$ bovine serum albumin and $3 \%$ human serum albumin for at least $30 \mathrm{~min}$ utes at room temperature in a humidified box. Samples were subsequently incubated with phycoerytrin (PE)-conjugated monoclonal antibody to CD-31 (PECAM-1) in a 1:50 dilution (Pharmigen, San Diego, CA) to stain for microvessel density. After 1-hour incubation at room temperature, slides were washed with PBS and immediately imaged using an Olympus BX-60 fluorescence microscope at 200X magnification. Digital images were stored and processed using Adobe Photoshop (Adobe Inc., Mountain View CA).

To assess the extent of total cell death, tissue sections were stained by using the TUNEL (terminal deoxyribonucleotidyl transferase-mediated dUTP-nick-end labeling) assay, which was performed according to the manufacturer's instructions (in situ cell death detection kit, fluorescein; TUNEL, Roche). Although the TUNEL assay detects apoptosis, it can not be ruled out that TUNEL will also stain for necrosis, where extensive DNA fragmentation may occur. Stainings were imaged using an Olympus BX-60 fluorescence microscope at 200X magnification, and the digital images were stored and processed using Adobe Photoshop (Adobe Inc., Mountain View CA).

Quantification of microvessel density and the rate of total cell death were determined as described earlier. ${ }^{112}$ Statistical analysis was performed using the Student's t-test.

\section{Toxicity assessment}

As an indirect measure of toxicity, body weights of mice were monitored three times per week using a digital balance (Ohaus Florham, NJ). Hematacrit levels were determined in blood samples extracted by tail vein bleedings prior to treatment, ten days after the initiation and on the last day of treatment. Blood samples were collected using heparinized micro-hematocrit capillary tubes (Fisher; Pittsburgh, PA). Samples were spun down for 10 minutes in a micro-hematocrit centrifuge (Clay-Adams; NY) and hematocrit levels were calculated using an international microcapillary reader (IEC; Needham, Mass). To obtain creatinine levels, blood was withdrawn on the last day of treatment. Creatinine was determined according manufacturer's instructions (Sigma Diagnostics; St Louis, MO). 


\section{Results}

\section{Anginex inhibits tumor growth dose dependently}

The dose dependency of anginex was assessed in the MA148 human ovarian carcinoma xenograft model, whereby anginex was administered at $5 \mathrm{mg} / \mathrm{kg} /$ day, $10 \mathrm{mg} / \mathrm{kg} /$ day, and $20 \mathrm{mg} /$ $\mathrm{kg} /$ day via osmotic mini-pumps. For this study, there were three control groups: one given PBS containing BSA and the other two given PBS containing homologous peptide $\beta$ pep- 28 ( $5 \mathrm{mg} / \mathrm{kg} /$ day or $10 \mathrm{mg} / \mathrm{kg} / \mathrm{day}$ ). As a model for minimal residual disease, treatment was initiated immediately after inoculation of animals with tumor cells. Anginex was observed to inhibit tumor growth dose dependently with the optimal dose being $10 \mathrm{mg} / \mathrm{kg} /$ day (Figure 1). At this dose, anginex inhibited tumor growth by $80 \%$ relative to control groups. Whereas greater effectivity was not observed at the higher dose of $20 \mathrm{mg} / \mathrm{kg} /$ day, tumor growth was inhibited by only $50 \%$ on average at half the optimal dose $(5 \mathrm{mg} / \mathrm{kg} /$ day). Tumors in all control groups were essentially of the same size. Data, taken from three independent studies, are shown as average tumor volumes in $\mathrm{mm}^{3} \pm \mathrm{SE}$ (Figure 1).

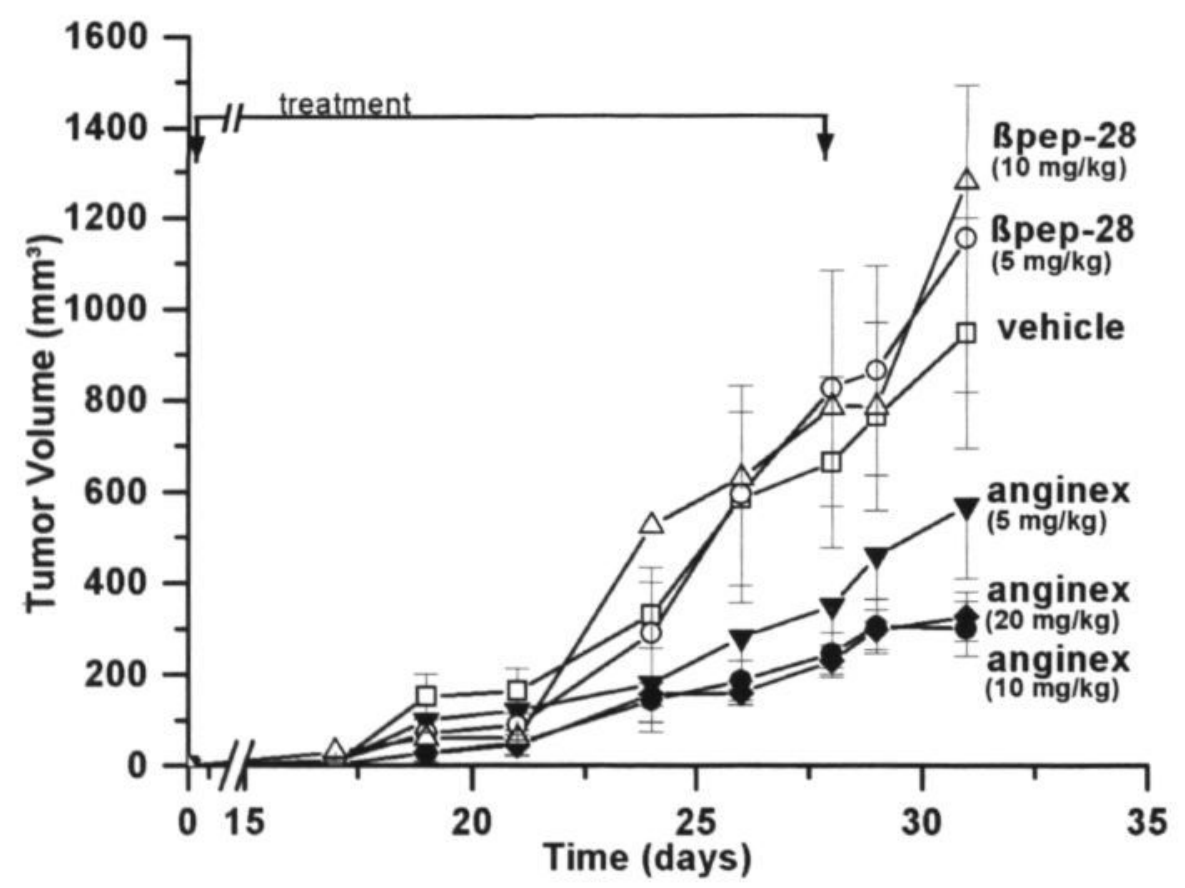

Figure 1. Anginex causes significant tumor growth inhibition. The mean tumor growth of human MA148 ovarian carcinoma is shown for athymic mice treated with a dose-range of anginex (closed triangle down: $5 \mathrm{mg} / \mathrm{kg} /$ day, $n=14$; closed circle: $10 \mathrm{mg} / \mathrm{kg} /$ day, $n=16$; closed diamond: 20 $\mathrm{mg} / \mathrm{kg} / \mathrm{day}, n=8$ ) administered via mini-pumps implanted in the left flank of animals. Controls contained PBS with BSA (open square; $n=13$ ) and PBS with Bpep- $285 \mathrm{mg} / \mathrm{kg} /$ day (open circle; $n=8$ ) and 10 $\mathrm{mg} / \mathrm{kg} / \mathrm{day}$ (open triangle up; $n=4$ ). The treatment period was initiated on the day of tumor inoculation (day 0 ) and lasted for 28 days as indicated by the horizontal arrow in the figure. Data were taken from three independent studies and represent the mean tumor volume in $\mathrm{mm}^{3}( \pm \mathrm{SE})$. 


\section{Anginex inhibits growth of established tumors}

Having demonstrated its in vivo efficacy, anginex was then tested in mice with established MA148 tumors (approximately 50 to $75 \mathrm{~mm}^{3}$ ). The optimal dose of $10 \mathrm{mg} / \mathrm{kg}$ / day was administered via mini-pumps, which resulted in a $70 \%$ to $80 \%$ inhibition of tumor growth (Figure 2A). The efficacy of anginex against MA-148 tumors was comparable to that observed against tumors produced using another ovarian carcinoma cell line, SKOV-3 (data not shown). As an alternative to continuous systemic administration by osmotic mini-pumps, anginex was encapsulated in alginate beads. Delivery of anginex via alginate beads was less pronounced compared to mini-pumps, producing only about a $50 \%$ reduction in tumor volume (Figure 2A). Lower efficacy was also observed when anginex was administered loco-regional by daily subcutaneous injections. At the sub-optimal dose of $5 \mathrm{mg} / \mathrm{kg} /$ day, tumor growth in these experiments was inhibited by only $30 \%$. Giving the same dose of anginex by twice daily injections did not improve efficacy of the peptide (Figure 2B).
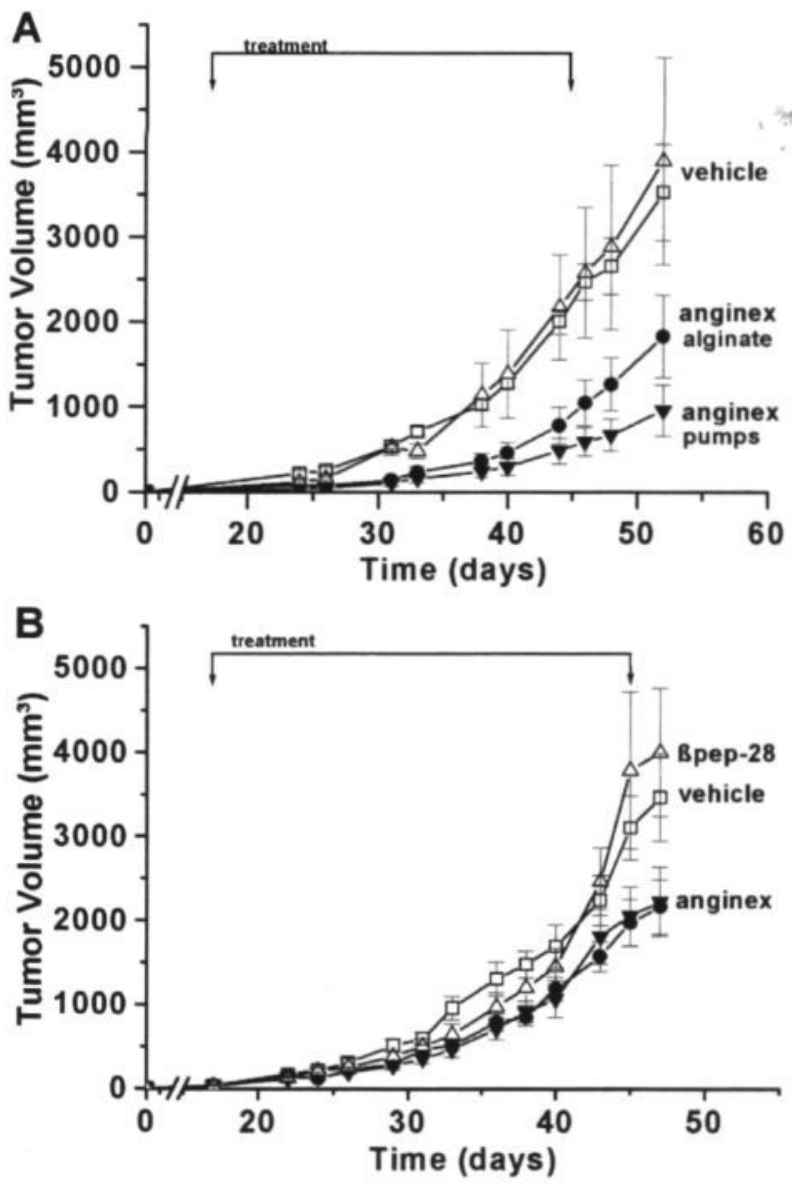

Figure 2. Effect of different routes of administration for anginex. (A) Tumor growth of human MA148 ovarian carcinoma in athymic mice treated with anginex (10 $\mathrm{mg} / \mathrm{kg}$ /day) that was administered in alginate beads (closed circle; $n=10$ ) or via osmotic mini-pumps (triangle down; $n=9$ ) implanted in the left flank of animals. Tumors grew on the right flank of animals. The alginate control group (open square) used 4 mice, whereas the mini-pump control group used 5 mice (open triangle up). (B) The mean tumor growth of human MA148 tumors in athymic mice treated with anginex (closed circle: $5 \mathrm{mg} / \mathrm{kg} /$ day or closed triangle down: $2.5 \mathrm{mg} / \mathrm{kg} /$ day b.i.d.) that was administered by daily loco-regional injection near the site of the tumor. Controls were performed using PBS with BSA (open square) and PBS with Bpep- 28 (open triangle up: $5 \mathrm{mg} / \mathrm{kg} /$ day). 10 mice were present in all groups except controls, which used 9 mice.

Horizontal arrows in both panels indicate the treatment period, which was initiated on day 17 post-inoculation when palpable tumors were present and lasted until day 45. Data have been taken from two independent studies and represent mean tumor volume in $\mathrm{mm}^{3}( \pm \mathrm{SE})$. 


\section{Anginex conjugation to HSA enhances inhibition of tumor growth}

Since anginex is a small peptide that may be cleared rapidly via filtration through the kidney, an attempt to improve bioavailability was made by conjugating anginex to human serum albumin (HSA-anginex, HSA:anginex molecule ratio of 1:5). Activity of the conjugate was first measured in EC proliferation assays and the results were comparable in both assays. On a molar basis, the activity of conjugated and unconjugated anginex, in these in vitro assays, was found to be essentially the same, whereas anginex is basically ineffective against the cancer cell line MA148 (Figure 3).

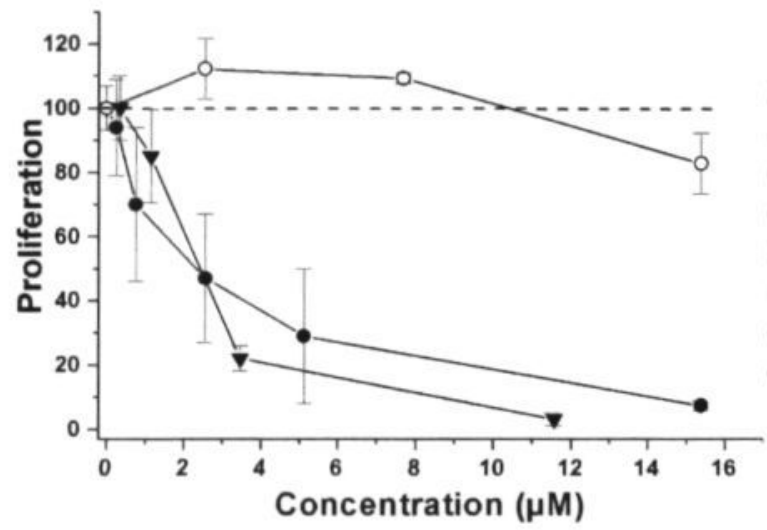

Figure 3. The effect of free anginex and conjugated anginex on HUVEC and human ovarian MA148 carcinoma. Proliferation data is shown which was measured after 72 hours of culture in the presence of free anginex (open circle, on MA148; closed circle, on EC) or conjugated anginex (closed triangle down, on EC) in different concentrations.

Figure 4. The mean tumor growth plotted for human MA148 ovarian carcinoma in athymic mice. Treatment lasted for 28 days and a prolonged significant tumor growth reduction is seen until termination of the study $(* P<0.05)$. The insert shows the data during treatment. Treatment consisted of anginex (closed circle; $2.5 \mathrm{mg}$ / $\mathrm{kg} /$ day; $n=11$ ) or HSAconjugated anginex (closed triangle down; $41.5 \mathrm{mg} / \mathrm{kg} / \mathrm{d} ; n=$ 18) that was administered via osmotic mini-pumps implanted in the left flank of animals. Tumors grew on the right flank of animals.

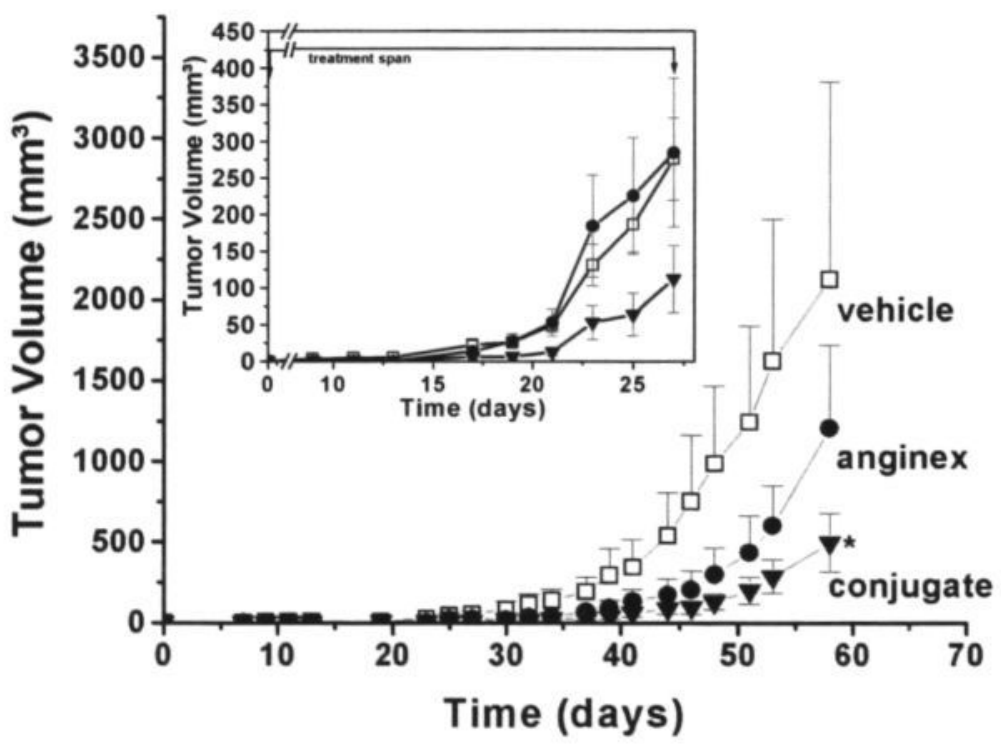

Control animals were treated with free HSA in PBS (open square; $n=17$ ). The horizontal arrow indicates the treatment period, which was initiated on the same day as inoculation (day 0; prevention model). Data were pooled from three independent studies and represent mean tumor volume in $\mathrm{mm}^{3}( \pm \mathrm{SE})$. 
In vivo activity of the conjugate was then tested in the MA148 xenograft tumor model along with unconjugated anginex. Molar equivalent doses (anginex at $2.5 \mathrm{mg} / \mathrm{kg} /$ day and the HSA-anginex conjugate at $41.5 \mathrm{mg} / \mathrm{kg} / \mathrm{day}$ ) were subcutaneously administered via osmotic mini-pumps. Data in Figure 4 show averages of three independent experiments. A significant improvement in tumor growth inhibition was observed when animals were treated with the conjugate. 30 days posttreatment (day 58), tumors in animals treated with HSA-conjugated anginex remained significantly smaller than those in control animals treated with free $\mathrm{HSA}(P<0.05)$. At this time point, the mean tumor volume in HSA-conjugate treated animals was approximately $75 \%$ less than in control treated animals. Results from tumor volume measurements were virtually the same as those derived from wet weights of post-mortem excised tumors (vehicle $2875 \pm 1404 \mathrm{mg}$; conjugate $767 \pm 267 \mathrm{mg}$ ).

\section{Toxicity assessment}

In vivo toxicity of anginex and its conjugate was assessed by observing animal behavior, determining body weight, measuring hematocrit and creatinine blood levels, and examining macroand microscopic morphology of internal organs upon autopsy at the end of each study. By any of these criteria, neither anginex nor its HSA-conjugate appeared to be toxic. Treated and untreated mice behaved and ate normally. Body weights of treated and untreated mice were the same among any of the groups studied. Each mouse gained on average approximately 3 grams during the course of the studies. In addition, prior to the start of treatment, ten days after the initiation of treatment, and the last day of treatment, blood was drawn and hematocrit levels were determined as measurement for bone marrow toxicity. Prior to treatment, the average percentage of red blood cells was $54.3 \% \pm 4.7 \%$. Ten days after initiating treatment, red blood cells accounted for $51.0 \% \pm 3 \%, 51.0 \% \pm 2 \%$ and $46.3 \% \pm 6.6 \%$ for control, anginex and conjugate treated groups, respectively. On the last day of treatment red blood cells accounted for $50.3 \% \pm 1.1 \%$, $50.0 \% \pm 2.6 \%$ and $50.0 \pm 1.0 \%$ for control, anginex and HSA-conjugate treated groups, respectively. Creatinine levels, which were determined on the last day of treatment, showed amounts of $49.6 \mu \mathrm{moles} / \mathrm{l} \pm 2.5 \mu \mathrm{moles} / \mathrm{l}, 46.6 \mu \mathrm{moles} / \mathrm{l} \pm 4.5 \mu \mathrm{moles} / \mathrm{l}$, and $42.1 \mu \mathrm{moles} / \mathrm{l} \pm 3.0 \mu \mathrm{moles} / \mathrm{l}$ for control, anginex and conjugated treated groups, respectively. At the termination of all studies, macro- and microscopic morphology of internal showed no abnormalities among all groups of animals. 

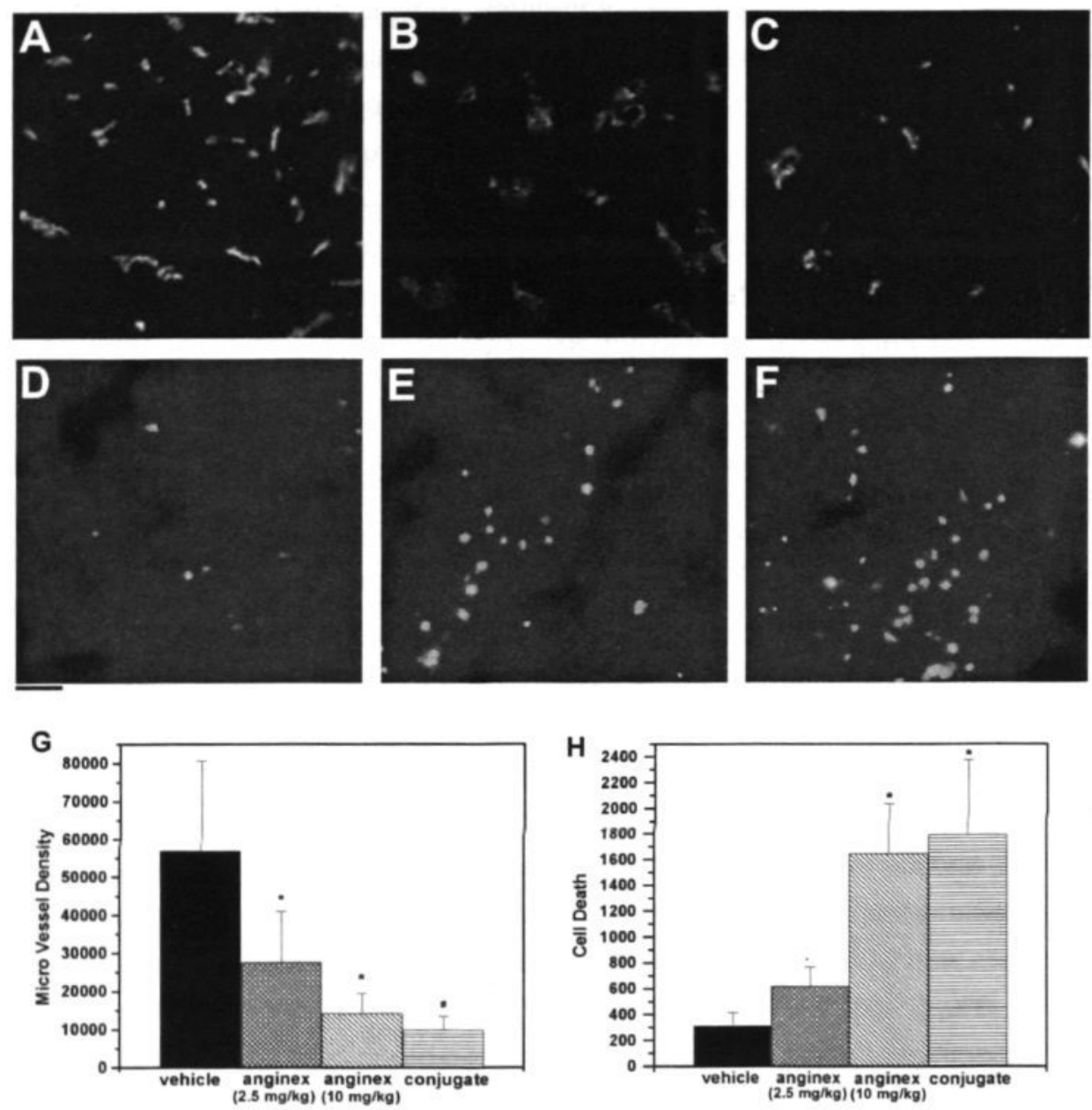

Figure 5. Histochemical analysis. Tumor cross-sections were stained for vessel density (panels A, B, C) and for apoptosis (panels D, E, F). Microvessel density (MVD) is revealed by PE-labelled anti-CD31 antibody staining. Apoptosis staining is revealed by using the TUNEL assay. Panels A and D exemplify tumor tissue cross-sections taken from control-treated mice; $\mathrm{B}$ and $\mathrm{E}$ exemplify tumor tissue cross-sections taken from anginex-treated mice, and C and $\mathrm{F}$ exemplify tumor tissue cross-sections taken from HSA-conjugate-treated mice. Data in panels $\mathrm{G}$ and $\mathrm{H}$ show the mean of white pixels per image ( \pm SE), MVD and apoptosis, respectively. Black bars represent results from control tumors; double diagonally striped and the left diagonally striped bars represent tumors treated with anginex (respectively $2.5 \mathrm{mg}$ / $\mathrm{kg} /$ day and $10 \mathrm{mg} / \mathrm{kg} /$ day), whereas horizontally striped bars represent tumors treated with HSA-conjugated anginex at $41.5 \mathrm{mg} / \mathrm{kg} /$ day. Statistical analysis was performed using the Student's t-test, where the asterix indicates $P<0.05 \mathrm{vs}$. control-treated tumors and the symbol \# indicates $P<0.01$ vs. anginex-treated $(2.5 \mathrm{mg} / \mathrm{kg})$ tumors. Original magnification X200; scale bar $=50 \mu \mathrm{m}$. 
Table 1. Vessel density quantification and related parameters

$\begin{array}{lcccc} & \text { Vessel Density }^{\mathrm{a}} & \text { End Points }^{\mathrm{b}} & \text { Branch Points }^{\mathrm{c}} & \text { Vessel Length }^{\mathrm{d}} \\ \text { Vehicle } & 56785 \pm 23790 & 20.3 \pm 10.2 & 17.1 \pm 9.9 & 22.7 \pm 7.4 \\ \text { Anginex } & 27576 \pm 13304^{\circ} & 41.2 \pm 14.0 & 13.0 \pm 8.9^{\circ} & 7.2 \pm 3.0^{\circ} \\ \text { Conjugate } & 9875 \pm 3638^{\circ .1} & 30.4 \pm 16.6 & 3.2 \pm 2.3^{\circ, t} & 5.7 \pm 2.7^{\circ .1}\end{array}$

a Following binarization of images, microvessel density was estimated by scoring the total number of white pixels per field. Results show the mean white pixel count per image \pm standard deviation.

'Mean number of vessel end points \pm standard deviation as determined after skeletonization of the images.

${ }^{c}$ Mean number of vessel branch points/nodes per image in pixels \pm standard deviation as determined after skeletonization of the images.

${ }^{d}$ Mean total vessel length per image in pixels \pm standard deviation as determined after skeletonization of the images.

- $P<0.05$. Experimental group compared to vehicle, using the Student's t-test.

' $P<0.01$. Experimental group compared to anginex, using the Student's t-test.
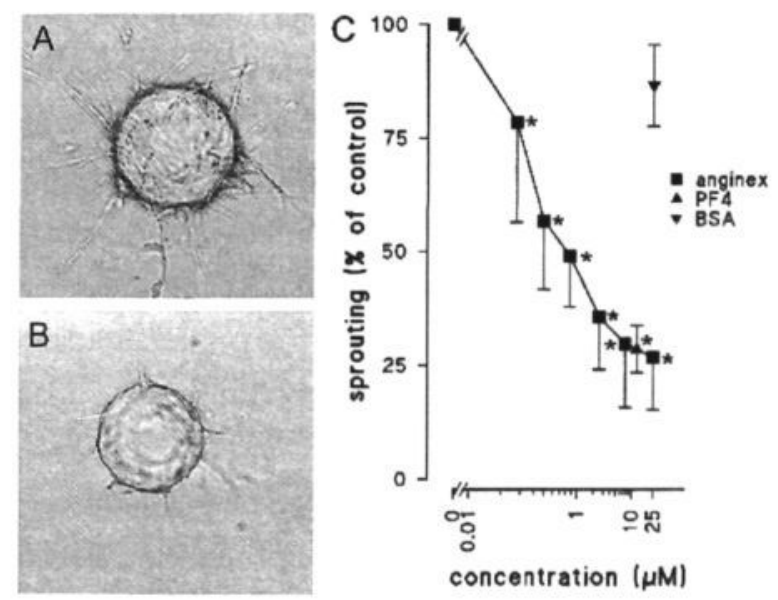

Figure 6. Anginex inhibits in vitro angiogenesis in a collagen matrix. BCE were cultured on gelatin coated Cytodex-3 beads in a collagen matrix. Sprouting was induced by addition of $20 \mathrm{ng} / \mathrm{ml}$ bFGF (A). (B) Sprouting of BCE in the presence of 20 $\mathrm{ng} / \mathrm{ml}$ bFGF and $25 \mu \mathrm{M}$ anginex. The beads have a mean size of $170( \pm 40)$ microns. Quantification of results (C) was performed by NIH-image software $\left(n=3,{ }^{*} P<0.013\right)$.

\section{Aspects of the mechanism of action}

To demonstrate that anginex functions by inhibition of blood vessel formation, microvessel density (MVD) and the extent of total cell death (Figure 5 and Table 1) in cross sections from tumor tissues were determined. Results show that anginex inhibits the number of microvessels by up to $50 \%$, whereas the conjugate inhibited MVD by approximately $80 \%$. Not only was the number of microvessels reduced in anginex treated animals, but vessel length was also reduced by a similar extent. This, together with an increase in the number of endpoints, is indicative of a change in vessel architecture. In all parameters (Table 1), the HSA-conjugate demonstrated greater 
anti-angiogenic effectivity compared to free anginex. The TUNEL assay, which reveals the extent of total cell death within tumors (Figure 5D-F), showed that cell death was greatest in animals treated with the HSA-anginex conjugate, consistent with results from microvessel staining (Figure $5 \mathrm{H})$. These results were supported by the inhibition of tube formation in the in vitro angiogenesis assay. In this assay, sprout formation was measured in a 3D collagen gel (Figure 6), and anginex was found to inhibit tube formation by $50 \%$ at $800 \mathrm{nM}$.

\section{Discussion}

Anginex is a rationally designed, cytokine-like peptide 33-mer, which has been shown in vitro to induce apoptosis specifically in angiogenically-activated endothelial cells (EC) by inhibiting EC from adhering to and migrating on the extracellular matrix. ${ }^{36}$ The present paper reports the in vivo efficacy of this small peptide and its development as a therapeutic anti-cancer agent. We have shown here that anginex significantly inhibits the growth of ovarian carcinoma-derived tumors in athymic mice. Of the three administrative routes used (daily loco-regional bolus injections, continuous systemic delivery via osmotic mini-pumps, and suspension in alginate beads implanted subcutaneously), continuous systemic administration of the peptide worked best. Inhibition of tumor growth was observed to be dose dependent and optimal at $10 \mathrm{mg} / \mathrm{kg}$, with a maximum reduction in tumor size of about $80 \%$ relative to control. When anginex was administered loco-regionally by once or twice daily injections, tumor growth was inhibited by only about $50 \%$. This reduction in efficacy is consistent with the previous observation that continuous administration of endostatin resulted in sustained systemic concentrations of the protein leading to increased efficacy, which was manifested as increased tumor regression compared to single daily bolus administration. ${ }^{107}$ Nevertheless, it was somewhat surprising to find that twice-daily injection of the same total dose did not lead to enhanced efficacy over single daily injections as was demonstrated with other angiogenesis inhibitors, e.g., matrix metallo proteinase inhibitor AG3340. ${ }^{113}$ AG3340 was able to inhibit tumor growth at the same rate as when it was administered in only $1 / 8$ of the dose, but injected multiple times a day. The plasma levels of the proteinase inhibitor explained this effect. Apparently, single or double daily injections of anginex did not produce appropriate threshold or steady state levels of the drug in the serum to be as effective.

As a third route of administration, we also investigated the use of alginate beads in which anginex was suspended. This non-toxic and biodegradable method is an established method in drug delivery 114 and was also reported to be an effective means of delivery by others in the antiangiogenesis field ${ }^{115}$. However, with anginex this slow-release approach was less effective than administration via osmotic mini-pumps. At best, tumor growth was inhibited by about $60 \%$ when delivered via alginate beads. The problem with the use of alginate beads stems from the fact that the percentage of encapsulated compound can differ and that administration, i.e., release, is not well regulated. ${ }^{116}$ 
Because anginex has a relatively low molecular weight $(3.9 \mathrm{kDa})$ and may, therefore, be rapidly excreted via the kidney, we thought that its in vivo efficacy could be improved by conjugating the peptide to a larger 'carrier' protein, i.e., human serum albumin (HSA;67 kDa). Molecular weight-dependent tissue accumulation and clearance has been systematically investigated for dextrans ${ }^{117}$ and polyethyleneglycols (PEGs) ${ }^{118}$. For PEGs it was found that the circulatory half-life of PEG increased from $18 \mathrm{~min}$ to 1 day as the PEG molecular weight increased from $6 \mathrm{kDa}$ to $190 \mathrm{kDa}$. However, although renal clearance decreased, hepatic clearance increased with increasing PEG molecular weight. For dextrans in a molecular-weight range of $4 \mathrm{kDa}$ to $150 \mathrm{kDa}$, renal clearance decreased with increasing molecular weight, whereas hepatic clearance was maximal at $70 \mathrm{kDa}$ and a further increase in molecular weight resulted in a decrease of liver uptake. Conjugation of smaller molecules to larger carrier molecules, e.g. HSA, has been shown to be effective in increasing bioavialablity for reasons, beyond an increase in size, e.g., changes the molecular hydrophobicity. For instance this was demonstrated by conjugating the well-known and hydrophobic chemotherapeutic agent paclitaxel to HSA. ${ }^{119}$ Because anginex is readily soluble in water ${ }^{36}$, this latter point is moot with regards to explaining in vivo effects from the HSA-anginex conjugate. The HSA-anginex conjugate shows a substantial improvement in efficacy. Whereas a sub-optimal dose of free anginex proved to be ineffective, a molar equivalent dose of anginex conjugated to HSA inhibited tumor growth by about $65 \%$. Although we did not measure serum levels of anginex or of the HSA-anginex conjugate, treatment with the conjugate presumably led to prolonged circulation in the serum merely due to the increase in size. This, in turn, relates to improved bioavailability of the drug.

Mechanistically, anginex functions in vitro as an anti-angiogenic agent. ${ }^{36}$ Immunohistochemical results on cross-sections of exercised tumors from anginex-treated and untreated animals, demonstrate that tumor growth inhibition by anginex is indeed mediated by its ability to inhibit angiogenesis. Here, this has been quantified by microvessel density and digital analysis of vessel architecture. Microvessel density in tumors, but not in other tissues, is significantly decreased in anginex-treated animals compared to control tumors. In addition, anginex-treated tumors have smaller vessels and the analysis of vessel architecture reveals decreased branching and less differentiation of the vascular bed. Furthermore, TUNEL analysis shows an increased amount of cell death within tumors exposed to anginex. This increased cell death most likely results from a reduction in angiogenesis, i.e., vessel density reduction, because in vitro data demonstrate that anginex only affects angiogenically-activated endothelial cells (Figure 3 and Griffioen et $\left.a l .^{36}\right)$. Therefore, apoptosis of tumor cells is not a direct effect of anginex treatment, but rather it is an indirect effect due to a reduction in the number of endothelial cells in the tumor. In studies with other anti-angiogenics, similar observations were made. For example, treatment with C225, an antibody to the EGF receptor showed that inhibition of tumor-induced angiogenesis led to tumor cell apoptosis and regression which, in turn, led to a significant anti-tumor effect against human pancreatic carcinoma in nude mice. ${ }^{120}$ 
For several reasons, anginex shows promise for clinical use as a therapy against cancer. Anginex is a potent anti-tumor agent that demonstrates no signs of toxicity in mice. Moreover, the peptide is synthetically produced and has a very long shelf-life either as a powder or in solution. Currently, we are using the NMR structure of anginex and derived structure-function relationships to design a small-molecule mimetic of the peptide. 


\title{
Chapter 3
}

\section{The designer anti-angiogenic peptide anginex targets tumor endothelial cells and inhibits tumor growth in animal models}

\author{
Daisy W.J. van der Schaft, Ruud P.M. Dings, Quido G. de Lussanet, \\ Loes I. van Eijk, Annemiek W. Nap, Regina G.H. Beets-Tan, \\ Jessica C.A. Bouma-Ter Steege, John Wagstaff, Kevin H. Mayo and \\ Arjan W. Griffioen
}

FASEB J. 2002; 16(14): 1991-3. 


\section{Abstract}

The de novo designed angiogenesis inhibitor anginex was tested in vitro and in vivo for its mechanism of action and anti-tumor activity. The data presented here demonstrate that anginex is a powerful anti-angiogenic agent with significant anti-tumor activity. The mechanism of action of anginex was found to be the induction of anoikis leading to apoptosis in angiogenically activated endothelial cells, resulting in an up to $90 \%$ inhibition of migration in the wound assay. Anginex inhibited angiogenesis as demonstrated in the in vitro mouse aortic ring assay. In addition, tumorinduced angiogenesis in the chick chorioallantoic membrane was markedly inhibited. Anginex showed profound anti-tumor activity in the syngeneic mouse B16F10 melanoma model and in a xenograft human tumor model. Microvessel density determination as well as magnetic resonance imaging showed that the anti-tumor activity in these tumor models resulted from the anti-angiogenic activity of anginex. A complete absence of toxicity was observed in these models. The data presented here demonstrate that anginex is a promising agent for further clinical development. 


\section{Introduction}

Angiogenesis is crucial for numerous bodily functions, including normal processes such as embryogenesis and wound healing, and in pathological situations such as tumor growth, arthritis, restenosis, endometriosis, and diabetic retinopathy. ${ }^{10,121}$ The search for angiogenesis inhibitors, which could be of therapeutic use, has been mainly concentrated on controlling two of the processes promoting angiogenesis, namely endothelial cell (EC) growth and adhesion..$^{96,122}$ Targeting drugs to $E C$ is considered to be a potentially useful anti-cancer strategy primarily because EC are more accessible than other cells are to pharmacologic agents delivered via the blood and because EC are genetically stable and thus are not easily mutated into drug resistant variants. Most anti-angiogenic agents have been discovered by identifying endogenous molecules, primarily proteins, which inhibit EC growth. This traditional approach has produced several anti-angiogenic agents, such as platelet factor-4 (PF4) ${ }^{123}$, thrombospondin- $1^{124}$, interferon- $\gamma$ inducible protein- $10^{125}$, angiostatin ${ }^{8}$, endostatin ${ }^{9}$, vasostatin ${ }^{126}$, tumstatin ${ }^{54}$, and bactericidal permeability-increasing protein $(\mathrm{BPI})^{82}$. The use of anti-angiogenic agents in in vitro and in vivo studies, particularly those applied to anti-cancer research, has strongly suggested that anti-angiogenic therapy will be a promising therapeutic modality in the future, either as monotherapy or in combination with conventional cancer therapies.

Whereas several anti-angiogenesis molecules have been tested clinically, the majority of them possessed either significant toxicity or lacked sufficient anti-cancer activity. The most succesful anti-angiogenic compounds as yet, at least judged by evaluation in preclinical testing, are the ones that directly act on EC and block either cell growth and/or motility. Examples of such agents are angiostatin, endostatin, and BPI. In the search for novel angiostatic molecules, we have designed a $\beta$-sheet forming 33 mer cytokine-like peptide, anginex, which is a potent inhibitor of angiogenesis and belongs to the aforementioned group of inhibitors. The design of anginex was recently described as being based on the sequence and three-dimensional structure of PF4, interleukin-8, and BPI. Anginex has been demonstrated to specifically inhibit EC growth and adhesion and was found to inhibit angiogenesis in vitro. ${ }^{36,77}$ The aim of the present study was to further investigate the mechanism of action of anginex and to assess its ability to inhibit tumor growth in mice. To that end, the peptide was tested in a syngeneic mouse tumor model (B16F10 melanoma) in C57BL/6 mice, as well as in a human xenograft ovarian carcinoma model. Mechanistic studies using in vitro and in vivo angiogenesis models demonstrate that anginex exerts its effect by angiogenesis inhibition and that anginex specifically targets tumor EC. In vivo studies indicate that anginex is a powerful anti-angiogenic and anti-tumor agent. 


\section{Materials and Methods}

\section{Cell cultures and reagents}

The murine EC cell lines SVEC4-10 (purchased from ATCC) and TME (kindly provided

by Dr. Duijvestijn ${ }^{127}$ ) were cultured in Dulbecco's modified Eagle's medium (DMEM) (Life Technologies, Breda, The Netherlands) supplemented with $10 \%$ fetal calf serum (FCS) (BioWhittaker, Verviers, Belgium), 2 mM HEPES (Serva, Heidelberg, Germany), $50 \mathrm{IU} / \mathrm{ml}$ penicillin (ICN, Aurora, OH), and $50 \mathrm{IU} / \mathrm{ml}$ streptomycin (Seva, Heidelberg, Germany), in gelatin (Merch, Darmstadt, Germany) coated tissue culture flasks (Costar, Corning, NY). The cell lines were subcultured 1:8. B16F10 melanoma cells were cultured in MEM with Hank's salts, $10 \%$ FCS, antibiotics, MEM-vitamins, glutamine, nonessential amino acids, sodium pyruvate, and $\mathrm{NaHCO}_{3}$. MA148 human ovarian carcinoma cells were cultured in RPMI1640 supplemented with 10\% FCS and penicillin/streptomycin as described previously. The cells were subcultured twice a week 1:5. Human umbilical vein-derived endothelial cells (HUVECs) were cultured in RPMI1640, supplemented with $20 \%$ human pooled serum and antibiotics and $2 \mathrm{mM}$ glutamine.

For some experiments, mouse EC were cultured from heart and lung tissues of C57BL/6 mice. The organs were removed, minced, and subsequently incubated in collagenase (Life Technologies) and dispase (Life Technologies) (both $1 \mathrm{mg} / \mathrm{ml}$ ) for $1 \mathrm{~h}$ at $37^{\circ} \mathrm{C}$. After $30 \mathrm{~min}$, DNAse (Sigma Aldrich, Steinheim, Germany) was added to the mixture. After incubation, the tissue was sieved and centrifuged at $1500 \mathrm{rpm}$ for $10 \mathrm{~min}$. The pellet was resuspended in culture medium (DMEM, 20\% FCS, 2 mM L-glutamine, 2 mM sodiumpyruvate, $20 \mathrm{mM}$ HEPES, 1\% nonessential amino acids, antibiotics, 1 IU/100 ml heparin, $100 \mu \mathrm{g} / \mathrm{ml}$ ECGS). The cells were seeded in $25-\mathrm{cm}^{2}$ culture flasks coated with fibronectin. After $1 \mathrm{~h}$ of adherence, the unattached cells were decanted and the adherent cells were then cultured under different conditions. Using FACS-analysis, EC were identified by indirect staining with phyco-erythrin labeled rat anti-mouse CD31 (Pharmingen, BD, Woerden, The Netherlands). On the basis of light scatter characteristics, residual CD31-positive monocytes/macrophages were excluded. With human tissues, a similar protocol leads to identification of EC, as confirmed by acetylated-LDL binding and expression of ICAM-2 and CD105. ${ }^{128}$

Anginex and the control peptide $\beta$ pep- 28 (a $91 \%$ homologous and $67 \%$ identical peptide without anti-angiogenic activity) were synthesized as described previously. ${ }^{36}$ For some experiments, anginex was coupled to Oregon Green-488 (MW 152, Molecular Probes, Leiden, The Netherlands). $2.5 \mathrm{mg}$ anginex ( $\mathrm{MW} 3800$, dissolved in $0.5 \mathrm{ml} \mathrm{H}_{2} \mathrm{O}$ ) was mixed with $0.1 \mathrm{mg}$ Oregon Green (dissolved in $100 \mu$ l of dimethyl sulfoxide (DMSO), 1:1 molar ratio), and incubated for $1 \mathrm{~h}$ at room temperature. Unbound Oregon Green and DMSO were removed by dialysis overnight against $0.9 \% \mathrm{NaCl}$. Labeled anginex was concentrated to a final concentration of 3 $\mathrm{mg} / \mathrm{ml}$ by centrifugation in $\mathrm{YM} 3$ columns (Centricon). 


\section{Flow cytometric analysis of anginex binding to HUVEC}

HUVEC were either fixed directly after isolation with $1 \%$ paraformaldehyde for $30 \mathrm{~min}$ at $20^{\circ} \mathrm{C}$ or cultured for 3 days on $1 \mathrm{mg} / \mathrm{ml}$ fibronectin-coated tissue culture flasks with or without bFGF. After culture, the cells were harvested by trypsinization and fixed in $1 \%$ paraformaldehyde. After fixation, the cells were washed twice in PBS/0.1\% BSA $0.01 \%$ sodium azide and subsequently incubated with $25 \mu \mathrm{M}$ anginex on ice for $2 \mathrm{~h}$. After incubation, cells were washed twice and incubated for $1 \mathrm{~h}$ with MARC-1 mouse monoclonal anti-anginex antibody (Bouma-Ter Steege et al., unpublished observations). The cells were then washed again and incubated in goat anti-mouse Ig-FITC (Dako, Glostrup, Denmark). Cells were then washed and fluorescence of 5000 cells was recorded for each sample on a FACScalibur (Beckton Dickinson). The experiment was repeated three times with different HUVEC isolations.

\section{Apoptosis measurement}

Cells were cultured for 3 days on a fibronectin coat in the presence or absence of different concentrations of anginex. After this period, the cells were harvested with trypsin ( $0.125 \%$ ) (DIFCO, Detroit, $\mathrm{MI}$ ) and fixed for $2 \mathrm{~h}$ in $70 \%$ ethanol at $-20^{\circ} \mathrm{C}$. The cells were subsequently centrifuged at $1500 \mathrm{rpm}$ for $5 \mathrm{~min}$ and resuspended in DNA extraction buffer ( 89 volumes $0.05 \mathrm{M} \mathrm{Na}_{2} \mathrm{HPO}_{4} \cdot 2 \mathrm{H}_{2} \mathrm{O}$, 10 volumes $0.025 \mathrm{M}$ citric acid, 1 volume $100 \%$ Triton X-100, pH 7.4) and incubated for $20 \mathrm{~min}$ at $37^{\circ} \mathrm{C}$. After incubation, propidium iodide (PI) (Brunschwig Chemie, Amsterdam, The Netherlands) was added at a final concentration of $20 \mu \mathrm{g} / \mathrm{ml}$, and the DNA profile was directly analyzed with the FACScalibur. ${ }^{36}$ For assessment of apoptosis in freshly isolated ECs, CD31 staining was performed before the incubation in $\mathrm{PI}$.

Caspase- 3 activity was demonstrated by a 20 -min incubation of cell lysates ( $10^{6}$ cells/ $500 \mu \mathrm{l}$ PBS, $1 \%$ Triton X-100) with $10 \mu \mathrm{M}$ fluorogenic caspase-3 substrate (Ac-DEVD-MCA, Alexis Biochemicals, San Diego, CA) ${ }^{129}$ in $50 \mathrm{mM} \mathrm{Tris/HCl,} \mathrm{pH} \mathrm{7.5,} 1 \mathrm{mM}$ DDT as described by Fujita et al.. ${ }^{130}$ Fluorescence was measured at $380 \mathrm{~nm}$ for excitation and $460 \mathrm{~nm}$ for emission. In some experiments, the caspase inhibitor z-VAD.FMK (Alexis Biochemicals) was used $(10 \mu \mathrm{M})$ to inhibit apoptosis in EC. ${ }^{131}$

\section{Proliferation measurement}

Cells were seeded at 3000 cells per well in a 96-well culture plate and allowed to adhere for $2 \mathrm{~h}$ to a fibronectin ( $1 \mathrm{mg} / \mathrm{ml}, 2 \mathrm{~h}$ at room temperature) coat. After adherence, the medium was replaced by medium with or without $10 \mathrm{ng} / \mathrm{ml} \mathrm{bFGF}$ and containing a concentration range of anginex. After 3 days, the culture was pulsed for $6 \mathrm{~h}$ with $0.3 \mu \mathrm{Ci}$ [methyl- ${ }^{3} \mathrm{H}$ ]-thymidine (Amersham Life Science's Hertogenbosch, The Netherlands) per well. Activity was measured using liquid scintillation. In each experiment, measurements were done in triplicate. 


\section{Migration measurement}

SVEC4-10 and TME cells were cultured in triplicate on a 1- $\mathrm{mg} / \mathrm{ml}$ fibronectin coat in a 24well tissue culture plate. Cells were grown for 3 days until confluence. When confluent, a wound was made in the well, using a blunt glass pipette. The medium was replaced with medium containing $0,0.75,2.5,7.5,25$, or $50 \mu \mathrm{M}$ anginex, or $50 \mu \mathrm{M} \beta$ pep- 28 , and at $0,2,4,6,8$, and 24 $\mathrm{h}$, the wound width was measured at four different predefined places. Statistical significance of observed differences was assessed using Student's t-test. Photographs were made using an inverted microscope and a Contax $167 \mathrm{mt}$ camera.

\section{Mouse aortic ring assay}

For this ex vivo angiogenesis assay, the thoracic aortas were excised from 8- to 10-wkold C57BL/6 mice and immediately placed into PBS. The aortas were cleaned from blood and surrounding tissues and cut into 1-mm-thick cross-sectional rings. Single rings were placed in the wells of a 96-well tissue culture plate. A mixture $(100 \mu \mathrm{l})$ of 8 volumes of Vitrogen-100 (Cohesion, Palo Alto, CA), 1 volume $7.5 \% \mathrm{NaHCO}$, and 1 volume 10 times concentrated $\alpha-$ MEM (Life Technologies). The gels with the aorta fragments were allowed to solidify at $37^{\circ} \mathrm{C}$ for $2 \mathrm{~h}$. Medium $(50 \mu \mathrm{l})$ (DMEM, 20\% FCS, $2 \mathrm{mML}$-glutamine, $2 \mathrm{mM}$ sodiumpyruvaat, 20 mM HEPES, $1 \%$ nonessential amino acids, antibiotics, $1 \mathrm{IU} / 100 \mathrm{ml}$ heparin, $100 \mu \mathrm{g} / \mathrm{ml}$ ECGS) was put on top of the gels, and the cultures were maintained at $37^{\circ} \mathrm{C}$ for up to 7 days. Different culture conditions were tested in triplicate. Medium was changed every 3 days. Vascular sprouting from each ring was examined daily using an inverted microscope. Photographs were taken on day 3 and 7 . The width of the tube formation area was measured at four different predefined places of the aortic ring.

\section{Chorioallantoic membrane assay}

Fertilized eggs of Lohman-selected White Leghorns were incubated for 3 days, as previously described ${ }^{12,132}$, at $37^{\circ} \mathrm{C}, 55 \%$ relative air humidity, while being rotated every hour. At day 3 , a rectangular window $(1 \times 1.5 \mathrm{~cm})$ was made in the shell. Albumin $(2 \mathrm{ml})$ was withdrawn through the blunt end of the egg. The window was covered with transparent tape to prevent dehydration, and the eggs were further incubated without rotation until day 10. At day 10, a silicone ring was placed on the chorioallantoic membrane (CAM), and inside this ring, daily treatments were applied (65 $\mu$ l of different concentrations of anginex or control peptide $\beta$ pep-28).

For analysis of tumor-induced angiogenesis in the CAM, 4-mm ${ }^{3}$ fragments of B16F10 melanoma tumors, grown subcutaneously for 10 days in C57BL/6 mice, were transplanted onto the 10-day-old CAM. Immediately before the transplantation, the CAM was slightly damaged by removing its upper peridermal layer by quick adhesion and removal of lens tissue paper. Starting $2 \mathrm{~h}$ after transplantation, $65 \mu \mathrm{l}$ of a $10 \mu \mathrm{M}$ solution of anginex was applied topically every day. At 
day 17, photographs of the area at six times magnification were taken using a Wild M8 stereomicroscope equipped with a Nikon F301 camera. The vascular density indices were stereologically analyzed by projection of four concentric rings on the image of the CAM and enumeration of intersections with blood vessels.

\section{Tumor models}

The animal experiments were approved by the local ethical review committee. At day 0 , 6-wk-old C57BL/6 mice (obtained from Charles River) were inoculated with $2 \times 10^{5}$ B16F10 cells (provided by Dr. J. Fidler, Houston, TX) subcutaneously on the right flank. On the left flank, an Alzet osmotic minipump (Durect, Cupertino, CA) was placed on the same day. The mini-pumps administered either saline $(0.9 \% \mathrm{NaCl}$ solution); $1.5,6$, or $12 \mathrm{mg} / \mathrm{kg} /$ day of anginex; or $12 \mathrm{mg} / \mathrm{kg} /$ day of the control peptide $\beta$ pep-28 or BSA, dissolved in saline, for 14 days. TNP-470 (AGM1470; kindly provided by Takeda Chemical Industries, Osaka, Japan), which was used as a positive control angiostatic compound, was administered by i.p. injections of $60 \mathrm{mg} / \mathrm{kg}$ every 2 days ${ }^{133}$ as of day 9 after inoculation of the cells. Between day 6 and 9 , the tumors became visible in all mice, and tumors were measured daily. Tumor volumes were calculated as follows: width ${ }^{2} \times$ length $\times 0.52$. Because we observed in the $\mathrm{B} 16 \mathrm{~F} 10$ melanoma model that the first mice died of the disease shortly after day 16 , the experiments were terminated on that day.

MA148 ovarian carcinoma tumors ${ }^{134}$ were grown subcutaneously in 8-wk-old nu/nu Swiss mice (Charles River) by injection of $2 \times 10^{6}$ cells in $100 \mu$ of serum-free medium. When tumors reached a volume of $50 \mathrm{~mm}^{3}$, treatment was started by placement of osmotic mini-pumps. Statistical differences in tumor growth curves were analyzed using the two-way ANOVA test.

\section{In vivo peptide kinetics}

In some experiments, B16F10 tumor-bearing mice (tumor size of $\sim 150 \mathrm{~mm}^{3}$ ) were anesthetized and injected i.v. with $300 \mu \mathrm{g}$ of Oregon Green-labeled anginex (in $100 \mu \mathrm{l} 0.9 \%$ $\mathrm{NaCl})$. Blood samples $(50 \mu \mathrm{l})$ were taken from these and control mice by orbital punction at 2, 5, $10,15,30,60,120,180$, and 240 min after injection (blood from another mouse was given back to sustain the blood volume in the mouse). The blood samples were allowed to clot for $2 \mathrm{~h}$, and serum was harvested. The sera were diluted 1:5 in $\mathrm{H}_{2} \mathrm{O}$ and put in duplicate in a 96-well plate. Fluorescence of the samples was measured on a fluorimeter and compared to a standard dilution range. After $240 \mathrm{~min}$, the mice were sacrificed, and kidney, lung, muscle, spleen, liver, and tumor tissues were extracted. Cryosections $(5 \mu \mathrm{m})$ of these tissues were prepared, and vessel staining was performed with a phyco-erythrin (PE)-labeled anti-mouse CD31 antibody (see microvessel density assessment). Digital images were made on a fluorescence microscope at $200 x$ magnification. Green (anginex-Oregon Green) and red (vasculature) fluorescence were analyzed using ISIS software. 


\section{Microvessel density assessment}

Serial cryosections $(5 \mu \mathrm{m})$ were put on organosilane-coated object slides, air-dried for $24 \mathrm{~h}$ at room temperature, and fixed with $1 \%$ paraformaldehyde (Sigma) for $1 \mathrm{~h}$. Endogenous peroxidase was blocked by incubation in $0.3 \% \mathrm{H}_{2} \mathrm{O}_{2}$ in methanol for $30 \mathrm{~min}$. Sections were blocked for nonspecific antibody binding with $5 \%$ BSA in PBS. The slides were incubated in biotinylated anti-mouse CD31 antibody (Pharmingen) for $1 \mathrm{~h}$. After incubation with avidine-biotin complex (Vector Laboratories) for $\mathbf{3 0} \mathrm{min}$, the slides were developed with diaminobenzidine (Sigma) and counterstained with haematoxylin (Merck), and the slides were mounted in entellan (Merck). The microvessel density (MVD) was evaluated as described previously. ${ }^{135}$ In short, two independent observers assessed MVD by counting of vessels in three high-power fields randomly selected within a section. In some experiments, standardized analysis of pixel density with Scionimage software was used for the quantification of MVD. ${ }^{12}$ Significance of observed differences were assessed using Student's t-test.

\section{Magnetic resonance imaging (MRI)}

Mice from the B16F10 melanoma model were selected for MRI. Mice were anesthetized (ketamine $100 \mathrm{mg} / \mathrm{kg}$ and xylazine $10 \mathrm{mg} / \mathrm{kg}$, s.c.), and MRI measurements were done with a 5cm-diameter high-resolution surface coil at 1.5 Tesla (ACS-NT, Philips Medical Systems, Best, The Netherlands). The MRI protocol included a $T_{2}$-weighted 2D-TSE anatomic acquisition (TR/ TE $3427 / 150 \mathrm{~ms}$, TF 25) and $T_{\text {- }}$-weighted 3D-FFE (TR/TE $50 / 7 \mathrm{~ms}$, flip $35^{\circ}$, voxel $0.5 \times 0.5 \times 2$ $\mathrm{mm}^{3}$ ) pre- and postcontrast measurements (Gd-DTPA $0.015 \mathrm{mmol} / \mathrm{kg}$; Magnevist, Schering, Berlin, Germany). Subtraction of the postcontrast minus precontrast measurements in the tumor reveals the residual contrast agent in tumor circulation and interstitial space. After the measurements, the mice were sacrificed and the tumors were frozen in liquid nitrogen for histological analysis. Vessels were stained with rat anti-mouse CD31 and peroxidase labeled goat anti-rat Ig. The MVD was evaluated as described previously. ${ }^{136}$

\section{Results}

\section{Anginex inhibits proliferation of and induces apoptosis in mouse ECs}

Proliferation of EC is key to the process of angiogenesis. In an earlier study, we described the growth inhibitory effect of anginex on cultured EC. These data demonstrated that this effect was EC specific (growth of normal fibroblasts and tumor cell lines was unaffected) and nonspecies specific. ${ }^{36} \mathrm{Here}$, the influence of anginex on proliferation of mouse EC was analyzed using the EC lines SVEC4-10 and TME. After 3 days of incubation with $75 \mu \mathrm{M}$ anginex, the proliferation was inhibited by $50 \%$ and $74 \%$, respectively, for these two cell lines. The effect is plotted against the effect of anginex on HUVEC, demonstrating the relative nonsensitivity of mouse EC at the 
level of proliferation inhibition (Figure $1 \mathrm{~A}$ ). The data shown are from cultures with $10 \mathrm{ng} / \mathrm{ml} \mathrm{bFGF}$. For both the mouse and human EC, similar responses were observed in the absence of bFGF.

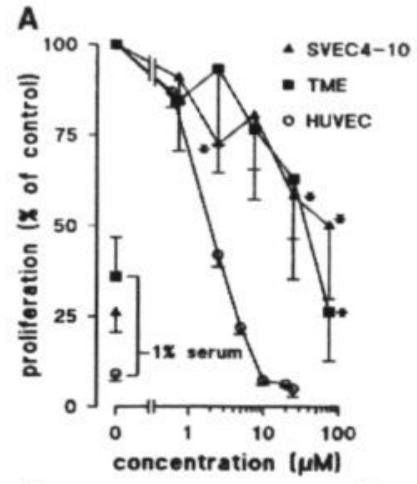

C

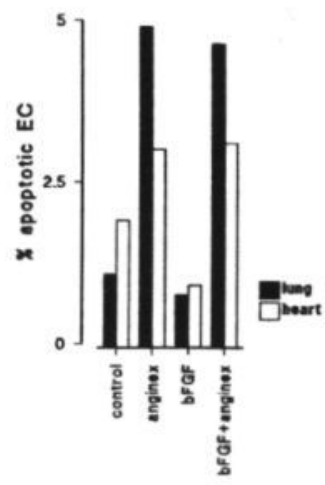

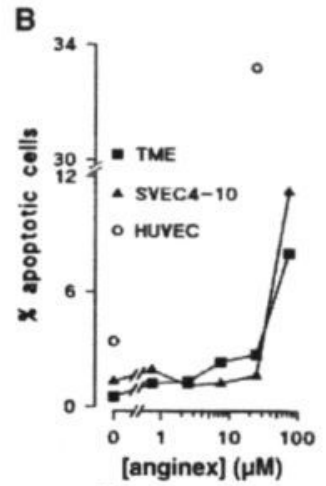

E

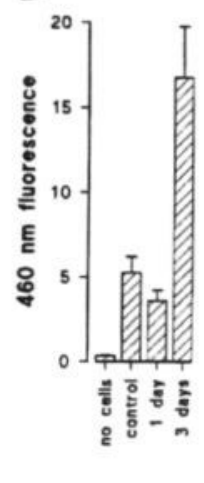

Figure 1. Anginex inhibits proliferation and induces apoptosis in endothelial cells.

(A) SVEC4-10, TME, and HUVEC were cultured for 3 days in the presence of a concentration range of anginex. Proliferation was measured by $\left[{ }^{3} \mathrm{H}\right]-$ thymidine incorperation. The mean data of four independent experiments $( \pm \mathrm{SE}$, ${ }^{*} P<0.05$ ) are shown. (B) Anginexinduced apoptosis as measured by assessment of subdiploid cells after DNA staining with propidium iodide. Results of one representative experiments out of four are shown. (C) Anginex induces apoptosis in EC isolated from mouse tissues. Single-cell suspensions were made from heart and lungs of C57BL/6 mice and cultured for 3 days on fibronectin-coated tissue culture plates in the absence or presence of $10 \mathrm{ng} / \mathrm{ml}$ bFGF and with or without $75 \mu \mathrm{M}$ anginex. Subdiploid propidium iodide staining cells after DNA extraction was determined in the CD31 expressing subset of cells using flow cytometry.

(D) 460-nm fluorescence of cleaved fluorigenic caspase-3 substrate Ac-DEVD-MCA, representing caspase3 activity, measured in samples without cells, samples of HUVEC cultures without anginex (control), and cells cultured for 1 or 3 days with $25 \mu \mathrm{M}$ anginex. (E) z-VAD.FMK was added to HUVEC cultures with or without anginex, and then subdiploid cells were analyzed on the flow cytometer. As a positive control, apoptosis was induced by starvation on low (1\%) serum.

To investigate whether growth inhibition of mouse EC was associated with the induction of apoptosis in these cells, flow cytometric analysis of DNA fragmentation was performed after culturing in the presence of anginex. Apoptosis induction by anginex in both TME and SVEC4-10 was observed to be concentration dependent, with maximal apoptosis induction in both cell lines found to occur at $75 \mu \mathrm{M}$ (Figure 1B). Again, HUVEC were more sensitive than mouse EC. When primary cultures of mouse $\mathrm{EC}$ isolated from lungs and hearts of mice and cultured for 3 days on a fibronectin coat, with or without bFGF, and in the presence or absence of $75 \mu \mathrm{M}$ anginex, similar results were observed. The freshly isolated EC cultured under normal conditions showed a cobble stone-like morphology. Upon exposure to anginex, cell detachment and apoptotic morphology were observed. The quantification of anginex-induced apoptosis in these EC is illustrated in Figure 1C. 
To confirm the finding that anginex induced apoptosis, we measured one of the early intracellular events that occurs during the induction of apoptosis, the activation of caspase-3. ${ }^{137}$ In HUVEC lysates that were treated for $72 \mathrm{~h}$ with anginex, significant caspase-3 activity was observed (Figure 1D). An inhibitor of caspase-3 (z-VAD.FMK) was able to completely inhibit anginex-induced apoptosis (Figure 1E). Moreover, z-VAD.FMK did not prevent the detachment of EC, suggesting that the induction of apoptosis by anginex was the result of cell detachment.
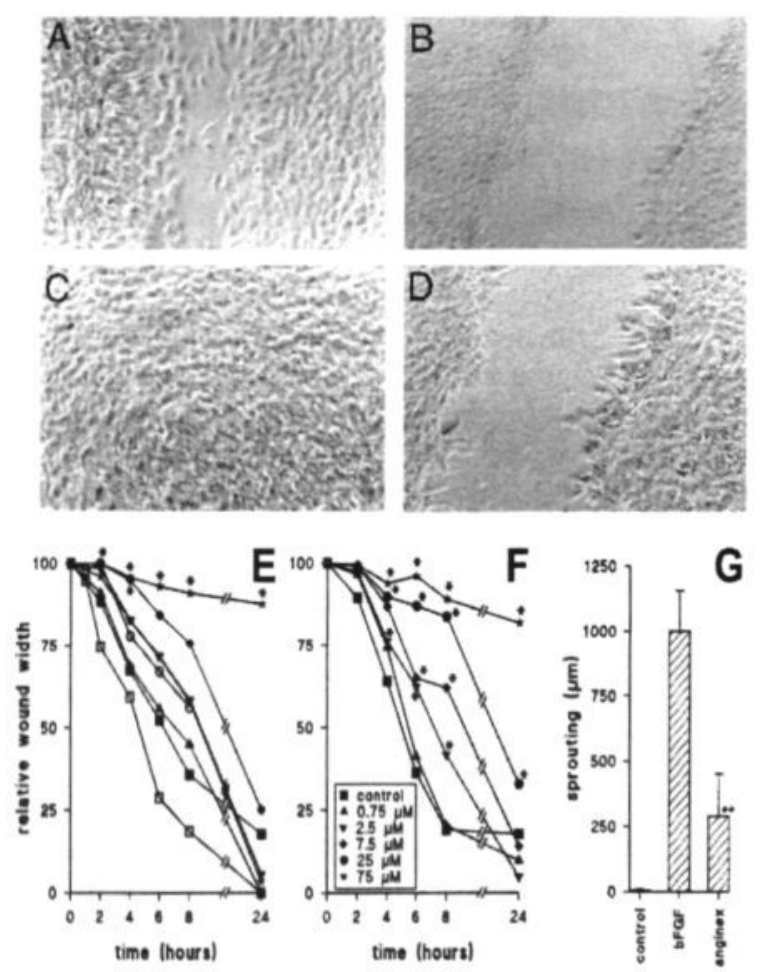

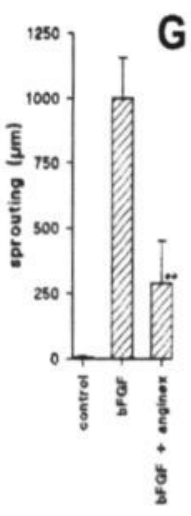

Figure 2. Anginex inhibits migration of EC. SVEC4-10 and TME cells were grown until confluence was reached, after which the monolayer was wounded and medium was replaced by medium with $10 \mathrm{ng} / \mathrm{ml}$ bFGF or medium supplemented with anginex in different concentrations. Control culture of TME at $8 \mathrm{~h}$ (A) and at $24 \mathrm{~h}(\mathrm{C})$ is shown. The same culture with $75 \mu \mathrm{M}$ anginex after $8 \mathrm{~h}$ (B) and after $24 \mathrm{~h}$ (D) is shown. The quantification of anginexinduced inhibition of TME migration (E) is shown. Open symbols represent HUVEC migration of control (open squares) and anginextreated (open circles) cultures. The migration inhibition in SVEC4-10 (F) is shown. The legend in $\mathrm{F}$ is also valid for $\mathrm{E}$ (squares, control; triangle up, $0.75 \mu \mathrm{M}$; triangle down, $2.5 \mu \mathrm{M}$; diamond, $7.5 \mu \mathrm{M}$; hexagon, $25 \mu \mathrm{M}$; asterisk, $75 \mu \mathrm{M}$ ). Results are presented as mean values of five different experiments. SE values are left out for the sake of clarity and were typically smaller than $10 \%(* P<0.05)$. The tube formation in the mouse aortic ring assay $(G)$ is shown. Mean values of a typical experiment out of four are shown $\left( \pm \mathrm{SE},{ }^{* *} P<0.001\right)$.

\section{Anginex inhibits the migration and tube formation of EC}

Aside from EC growth, migration also plays a key role in the process of angiogenesis. To explore the effect of anginex on EC migration, the wound assay was used. Addition of anginex to wounded confluent monolayers of SVEC4-10 and TME caused migration of these cells to be inhibited in a dose-dependent manner. Figures 2A-D show TME cultures of control (Figures 2A and $2 \mathrm{C}$ ) and anginex-treated (Figures $2 \mathrm{~B}$ and $2 \mathrm{D}$ ) cells, $8 \mathrm{~h}$ (Figures $2 \mathrm{~A}$ and $2 \mathrm{~B}$ ) and $24 \mathrm{~h}$ (Figures 2C and 2D) after wounding. After $24 \mathrm{~h}$ and at a concentration of $75 \mu \mathrm{M}$, anginex inhibited TME (Figure 2E) and SVEC4-10 (Figure $2 \mathrm{~F}$ ) migration for $91 \%$ and $88 \%$, respectively. The response of HUVEC is shown in Figure 2E (open symbols) to demonstrate the higher sensitivity of mouse EC relative to HUVEC at the level of EC migration. The control peptide $\beta$ pep-28 
demonstrated no effect on EC migration (data not shown). Tube formation of EC was studied using the mouse aortic ring assay. Approximately $70 \%$ inhibition of sprout formation in this ex vivo model was observed for anginex at $75 \mu \mathrm{M}$ (Figure $2 \mathrm{G}$ ).
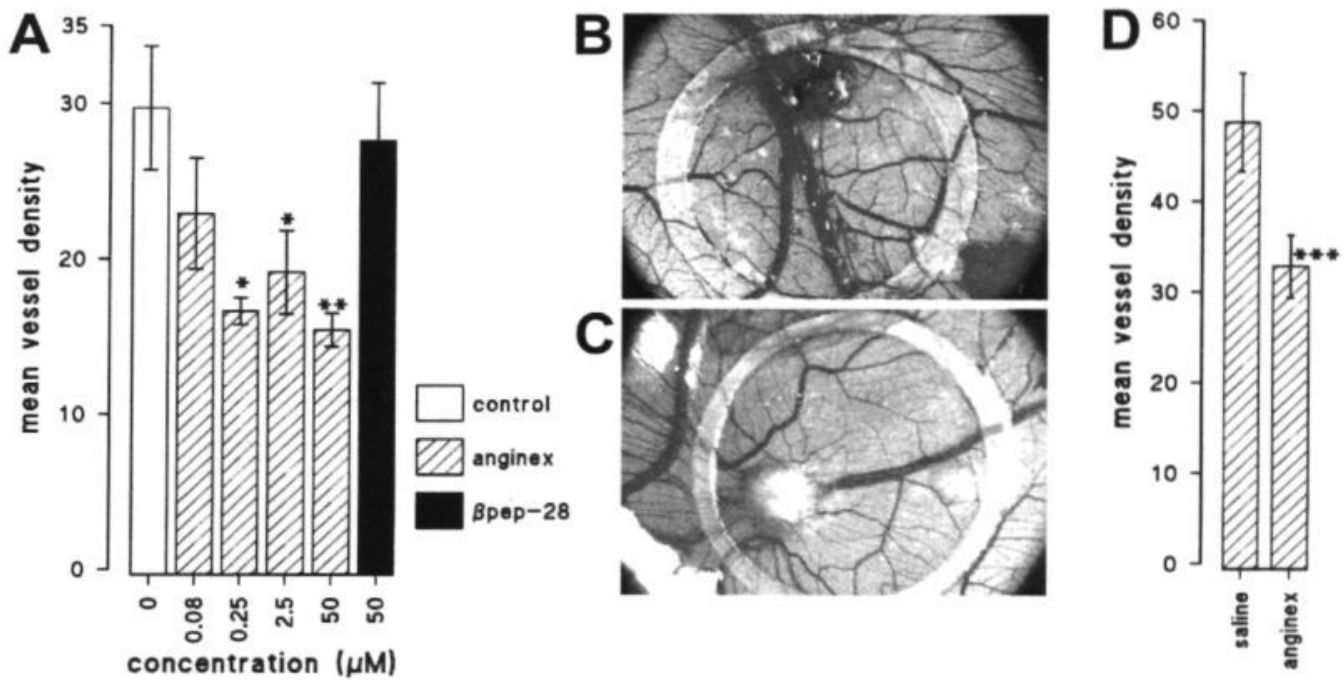

Figure 3. Anginex inhibits in vivo developmental and tumor-induced angiogenesis in the chorioallantoic membrane (CAM). A) The CAM was treated with saline and $0.075,0.25,2.5$, or 50 $\mu \mathrm{M}$ anginex. The homologous control peptide Bpep- 28 was tested at $50 \mu \mathrm{M}$. Mean vessel density results, expressed as mean number of intersecting vessels $/ 10 \mathrm{~mm}\left(n=6, \pm \mathrm{SE},{ }^{*} P<0.01, \quad{ }^{* *} P<0.005\right)$. B) Seventeen-day CAM with B16F10 melanoma present as of day 10 treated with saline $(0.9 \% \mathrm{NaCl})$. C) Seventeen-day CAM with B16F10 melanoma present as of day 10 topically treated daily with $65 \mathrm{ml}$ of a $10 \mu \mathrm{M}$ solution of anginex. D) Digital analysis and quantification of the mean vessel densities in control and anginex treated CAMs $(n=8, \pm \mathrm{SE}, * * * P<0.00002)$.

\section{Anginex inhibits angiogenesis in vivo}

To assay whether in vivo angiogenesis is perturbed by anginex, we used the CAM assay of the chick embryo. In the CAM assay, which is a model for developmental angiogenesis, topically added anginex inhibited the formation of new blood vessels in a concentration-dependent way. The maximal response of $\sim 50 \%$ angiogenesis inhibition was reached at $50 \mu \mathrm{M}$ anginex. Responses were already visible at $0.25 \mu \mathrm{M}$ concentrations, whereas treatment with the negative control peptide ßpep-28 showed no significant activity (Figure 3A). Tumor angiogenesis in this model was mimicked by transplantation of $4-\mathrm{mm}^{3}$ fragments of $\mathrm{B} 16 \mathrm{~F} 10$ mouse melanoma onto the CAM at day 10 . The melanoma tissue aggressively infiltrated into the CAM and efficiently induced angiogenesis (Figure 3B). The tumor tissue remained viable over the 7-day period of treatment, as determined by histochemistry (data not shown). This tumor-induced angiogenesis was inhibited by $33 \%$ after seven daily treatments with anginex $(P<0.00002)$ as measured on day 17 (Figures 3C and 3D). 


\section{Anginex inhibits tumor growth and angiogenesis in tumor models}

The anti-tumor effect of anginex was first examined in the B16F10 mouse melanoma model in C57BL/6 mice. Because this is a rapidly growing tumor model, treatment of mice, using various doses of anginex, was initiated at the time of inoculation with tumors cells. Tumors appeared in all experimental groups at roughly the same time, indicating that anginex did not affect the tumor take of B16F10 melanoma. A profound dose-dependent inhibition of tumor growth by anginex was observed at the end of treatment. At a dose of $6 \mathrm{mg} / \mathrm{kg} /$ day, administered by continuous subcutaneous infusion, using osmotic mini-pumps, tumor growth was significantly inhibited by $63 \%$ as compared to that in control mice. A maximal inhibition of $73 \%$ was observed at day 15 . Whereas treatment of mice with $12 \mathrm{mg} / \mathrm{kg} /$ day did not lead to a further significant increase in efficacy (64\% inhibition), a lower dose of $1.5 \mathrm{mg} / \mathrm{kg} /$ day inhibited tumor growth by only $36 \%$ (Figure 4A). TNP-470, used in a previously optimized treatment regimen ${ }^{138}$, was used in these experiments as positive control ( $60 \%$ inhibition) to demonstrate that angiogenesis inhibition can inhibit tumor growth in this model. Anginex treatment showed no toxicity in these animals as assessed by macroscopic and behavioral determinants and weight gain during the experiment, as well as by histological evaluation of the organs and measurement of hematocrit (data not shown).

To determine whether the tumor inhibitory effect by anginex was the result of angiogenesis inhibition, tumors were immunohistochemically screened for MVD using an anti-CD31 antibody. Anginex treatment resulted in a reduced vessel density in the tumor of $-50 \%$, relative to control tumors (Figures 4B and $4 \mathrm{C}$ ). A similar reduction in vessel density was found for tumors of TNP470 treated mice.

Magnetic resonance imaging (MRI) $T_{2}$-weighted anatomic acquisitions indicate the tumor site (Figures $4 \mathrm{E}$ and $4 \mathrm{~F}$ ). The subtraction of the postcontrast minus precontrast $T_{1}$-weighted measurements reveals a smaller signal intensity increase due to a lower concentration of residual contrast agent in the tumors of anginex-treated animals (Figure 4F, insert), compared to the signal intensity increase in tumors of control animals (Figure 4E, insert). For anginex-treated animals, the increase in signal intensity over the entire area of the tumor was $\sim 50 \%$ lower $(12.7 \%$ $\pm 3.9 \%$, mean $\pm \mathrm{SE})$ compared with that in control animals $(26.5 \% \pm 3.6 \%)$. Because the signal intensity did not differ significantly at the center of the tumor (anginex $12.5 \% \pm 4.7 \%$, control $17.0 \% \pm 4.5 \%$ ), this overall difference was the result of a significant reduction (46\%) in signal intensity at the rim of the tumor (anginex $17.3 \% \pm 4.3 \%$, control $32.0 \% \pm 3.4 \%, P<0.04$ ). 

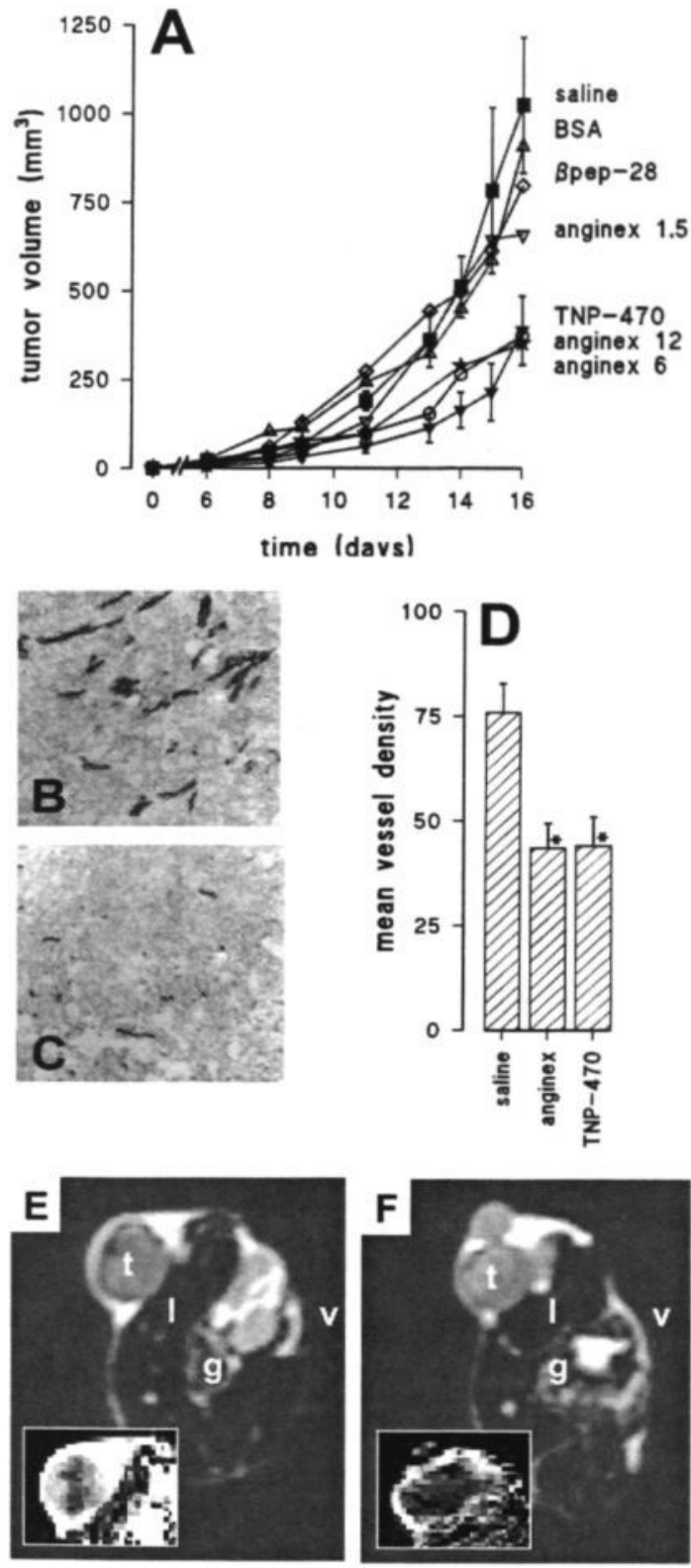

Figure 4. Anginex inhibits tumor growth in mice. (A) C57BL/6 mice were treated, directly after injection of B16F10 melanoma cells, with saline (solid squares) and 1.5 (open triangle down), 6 (solid triangle down), or 12 (open circles) $\mathrm{mg} / \mathrm{kg} /$ day doses of anginex or with $6 \mathrm{mg} / \mathrm{kg} /$ day BSA (open triangles up) or Bpep-28 (open diamonds) delivered by osmotic mini-pumps or with $2 \mathrm{mg}$ TNP-470 i.p. every 2 days as of day 9 (open asterisks). For clarity, variations in tumor measurements (SE was typically roughly $20 \%$ in all groups) are shown only for the anginex $6 \mathrm{mg} /$ $\mathrm{kg} /$ day and the saline groups. Statistical significant inhibition of tumor growth (two-way ANOVA test) was observed for anginex doses of $6 \mathrm{mg} /$ $\mathrm{kg} /$ day $(P<0.0001)$ and $12 \mathrm{mg} / \mathrm{kg} /$ day $(P<0.003)$ and with TNP-470 $(P<0.0001)$. Cryosections of tumors from control $(B)$ and $6 \mathrm{mg} / \mathrm{kg} /$ day anginex treated $(C)$ mice were stained with CD31 antibody for microvessel density (MVD) assessment. (D) Quantification of MVD as mean number of vessels per $100 \mathrm{~mm}^{2}( \pm \mathrm{SE}, * P<0.05)$. E) and F) $T_{2}$, weighted images (from mice with equally sized tumor volumes, indicated as $t$ ) of a BSA-treated control animal (E) and a anginextreated animal $(6 \mathrm{mg} / \mathrm{kg} /$ day $)(\mathrm{F})$. Subtractions of pre- and postcontrast $T_{1}$ - weighted tumor measurements of anginex and BSA-treated animals are shown in the inserts ( $\mathrm{g}$ means gut; 1 means left hind leg; $v$ means ventral side of the mouse).

To investigate the effect of anginex in a human tumor xenograft model, the slowly growing MA148 ovarian carcinoma model was tested in athymic mice ${ }^{134}$. In contrast to the B16F10 melanoma model, the effect of anginex treatment was studied on already established tumors. The treatment by subcutaneously placed osmotic mini-pumps was started on day 17 when tumors reached the size of $\sim 50 \mathrm{~mm}^{3}$. In this model a significant $(n=11, P<0.004) 75-80 \%$ inhibition of tumor growth was observed (Figure 5). MVD assessment in the tumor revealed a reduction of $52 \%$ in anginex-treated mice (data not shown). 


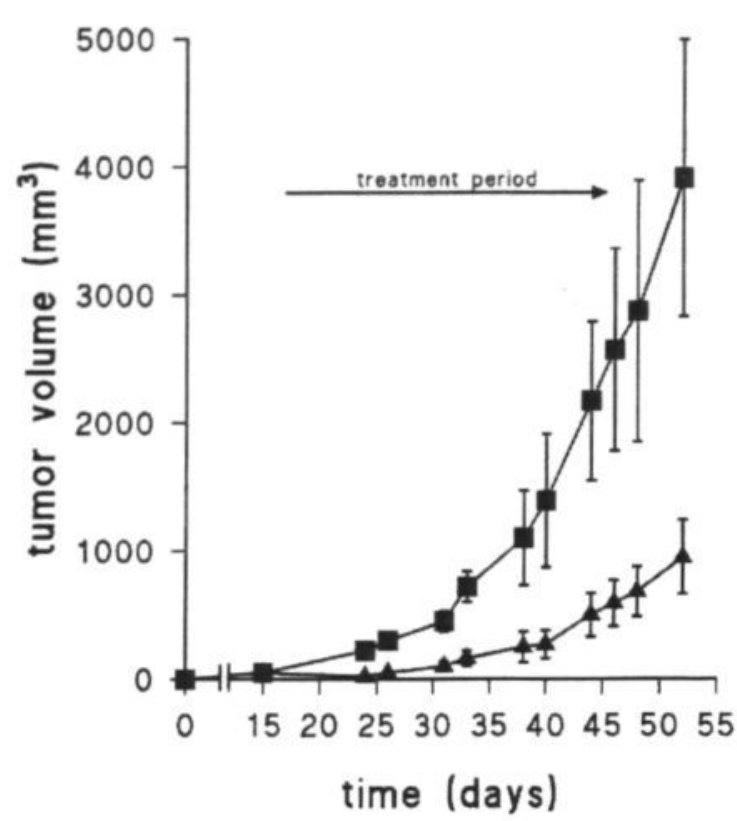

Figure 5. Anginex inhibits tumor growth in a human xenograft model. Mean tumor growth of MA148 human ovarian carcinoma in athymic mice treated with anginex (6 mg/kg, triangles) or BSA (squares), both dissolved in saline, administered by osmotic minipumps. Treatment (indicated by the arrow) was initiated at day 17 post-inoculation when tumors grew to approximately $50 \mathrm{~mm}^{3}$ in size. Results show mean tumor volumes of mice from two independent experiments $( \pm S E$, $n=11, P<0.004)$.

\section{Anginex targets angiogenically activated and tumor EC}

Injecting Oregon Green-labeled anginex i.v. in B16F10 tumor-bearing mice and measuring the serum of these mice at different time points revealed a serum half-life of anginex of $\sim 50 \mathrm{~min}$. After $1 \mathrm{~h}$, the Oregon Green-labeled anginex appeared in the urine, and after $4 \mathrm{~h}$, so much Oregon Green-labeled anginex was sequestered in the tumor that the fluorescence in the tumor was visible through the skin. In the tumors of mice sacrificed $4 \mathrm{~h}$ after the injection, Oregon Greenlabeled anginex clearly identified the tumor blood vessels (Figure 6E) but not the vessels of the hind limb muscle (Figure 6B). Immunohistochemical detection of blood vessels using a PElabeled anti-CD31 antibody (Figures 6A and 6D) proved that the Oregon Green-labeled anginex accumulated in the tumor vessels (Figure 6F) but not in vessels of the hind limb muscle (Figure $6 C)$. In the kidney, residual Oregon Green-labeled anginex was present in the tubuli, whereas blood vessels in the glomeruli as well as the peritubular blood vessels were completely devoid of anginex fluorescence (data not shown).

In an attempt to find in vitro evidence for the preference of anginex to affect activated EC, anginex binding was studied on resting HUVEC (directly fixed after isolation from the umbilical cord), HUVEC activated by culture conditions and HUVEC additionally activated by an angiogenic factor. A clear augmentation of binding, demonstrated by flow cytometry, using MARC-1 antianginex antibody, was observed with increased EC activation (Figure 6G). To obtain functional evidence for this, we attempted to bring $E C$ in a quiescent state. It was difficult to use protein and RNA synthesis inhibitors such as cyclohexamide and actinomycin-D because of the high sensitivity of HUVEC. When the cell cycle in HUVEC was blocked by culturing in the presence of excess 
thymidine, a process that synchronizes EC in early S phase of cell cycle, a clear reduction of apoptosis induction by anginex was observed (Figure $6 \mathrm{H}$ ).
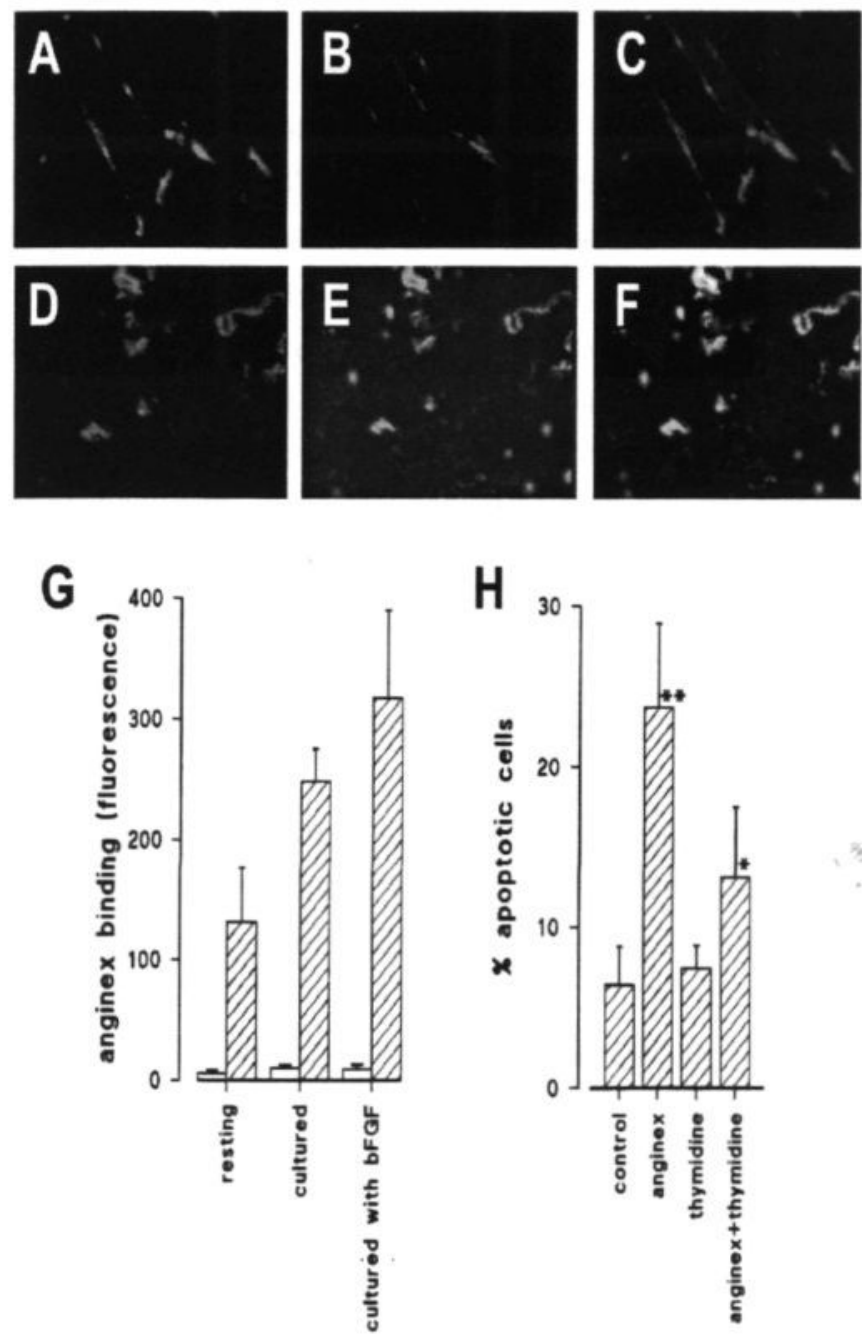

Figure 6. Anginex targets angiogenically activated tumor EC. B16F10 tumor-bearing mice were injected with $300 \mu \mathrm{g}$ of Oregon Green-labeled anginex. After 4 hours, the animals were sacrificed and organs were taken out. Cryosections $(5 \mu \mathrm{M})$ of muscle (A-C) and tumor (D-F) tissues were prepared and stained with PE-labeled CD31 antibody. Vessel staining ( $A$ and $D$ ) as well as Oregon Green Fluorescence (B and E) were recorded. The merged images ( $\mathrm{C}$ and $\mathrm{F}$ ) demonstrated the yellow double labeling of tumor but not muscle microvessels. (G) Enhanced anginex binding to HUVEC in higher activation stages. Anginex is identified by flow cytometry, using MARC1 anti-anginex mouse monoclonal antibody. Bars at the left of each set show results of control staining of the HUVEC in the absence of anginex. $(\mathrm{H})$ Decreased anginex activity (apoptosis induction) in HUVEC arrested in cell cycle by a double thymidine block. ** Significant difference with control EC $(P<0.05) ; *$ Significant decrease in apoptosis induction as compared with anginex culture without thymidine $(P<0.01)$.

\section{Discussion}

The aim of the present study was to assess whether the novel and unique approach of the de novo design of $\beta$-sheet scaffold cytokine-like peptides can be used to develop angiogenesis inhibitors that inhibit tumor growth in vivo. The current data demonstrate in both a syngeneic melanoma model as well as in a human xenograft model that anginex targets angiogenically activated tumor EC and effectively inhibits tumor growth in these models. 
In vitro studies suggest that angiogenesis inhibition by anginex is mediated by inhibition of migration in activated EC, ultimately leading to induction of apoptosis. We favor this view, rather than direct induction of apoptosis and subsequent detachment of EC from the matrix, because the caspase inhibitor z-VAD.FMK completely blocked the process of apoptosis but did not prevent the detachment and anoikis of the EC. Further evidence for this view was provided by the fact that the effect of anginex on migration was seen earlier than the effect on apoptosis ${ }^{36}$ and at concentrations that were not inhibitory for the proliferation of EC. These observations suggested that modulation of EC migration is the initial event, ultimately resulting in modulation of proliferation and induction of apoptosis. The mechanism of this effect is suggested to be the down-regulation of adhesion molecules for matrix components, as has previously been demonstrated in human $E C$ for $\alpha_{v} B_{3}$ - and $\beta_{1}$-intergrins and CD44. ${ }^{36}$

In the in vitro experiments, a small but notable difference was observed in the sensitivity for anginex between mouse and human EC. At the level of EC growth, measured in both proliferation and apoptosis assays, human EC were more sensitive for anginex than mouse EC. Interestingly, at the level of migration processes, mouse EC were found to be more sensitive to exposure to anginex. These differences might be explained, next to the species difference, by the fact that HUVEC are fresh nontransformed normal cells, whereas the mouse EC used were SV40 large-T transformed cell lines that may have acquired altered functional characteristics. Because the mouse vs. human differences do not show a consistent benefit for either EC type, the mouse model is still considered an appropriate model for preclinical testing of anginex.

Several in vivo angiogenesis models are available to test new compounds for anti-angiogenic or angiogenic activity (reviewed by Jain et al. ${ }^{139}$ ). The first model chosen here was the chick embryo CAM assay, which is an affordable and straightforward model that is ideally suited for initial screening of potential angiostatic compounds. In this assay, anginex effectively inhibited angiogenesis in a dose-dependent manner. The major drawback of the CAM assay in oncological research is, however, that it is a model for developmental angiogenesis, which may not reflect the processes involved in tumor angiogenesis. The xenogenic transplantation of mouse tumor tissue onto the CAM, however, clearly induced new blood vessel formation in addition to the developmental angiogenesis. Because anginex inhibited the tumor-induced angiogenesis to the level of vessel density in CAMs without a tumor, it is tempting to speculate that only the enhancement by the tumor is inhibited by anginex. However, because anginex also inhibits developmental angiogenesis, it is suggested that in this model anginex partially blocks both types of angiogenesis induction.

Because the CAM model demonstrated a clear in vivo inhibition of angiogenesis by anginex, tumor models were used to test both anti-angiogenic and anti-tumor activities. Initial 
studies indicated that administration of anginex by osmotic minipumps gave optimal results compared with loco-regional subcutaneous injections at the site of the tumor, once or twice daily subcutaneous injections or slow-release alginate bead strategies. In the syngeneic murine B16F10 melanoma model in C57BL/6 mice, a visible tumor mass is present at day 7-9 and the tumor reaches a maximal size after 16 days. Anginex demonstrated a profound anti-tumor effect in this aggressive tumor model. Because of the aggressiveness of the B16F10 melanoma, treatment had to start as early as possible. Starting treatment at the day of inoculation of the tumor is often regarded as an inappropriate model for advanced cancers, although it may be seen as a useful model for minimal residual disease. The major problem with this approach is that the compound tested is affecting tumor-take rather than inhibiting tumor growth. To circumvent this discussion, we switched to the use of a slower growing tumor, which provided the possibility of starting the treatment with already established tumors. In addition, a human xenograft model was chosen because of the future development of anginex for clinical use. An even higher efficacy of anginex, resulting in an up to $80 \%$ inhibition of tumor growth, was observed in this model.

The anti-tumor activity of anginex is most likely mediated by inhibition of angiogenesis because MVD values in anginex-treated tumors of both models were significantly decreased. The fact that anginex resembled the well-defined and generally accepted angiostatic activity of TNP-470, in both tumor growth and MVD measurements, is an additional indication of angiostasis by anginex. The results from the MRI experiment showed a reduced signal intensity in tumors of anginex treated animals compared with tumors in control animals. Areas of high signal intensity increase are known to have a high MVD and vascular permeability ${ }^{136}$ and are therefore considered areas with high angiogenic activity. These MRI results therefore support the conclusion that anginex inhibits tumor growth by inhibition of angiogenesis and lend support to the idea that MRI may be a useful noninvasive surrogate marker for angiogenesis inhibition in cancer patients.

The experiments with fluorescently labeled anginex (a bolus injection of $300 \mu \mathrm{g}$ in the tail vein) revealed a short serum half-life of anginex of $\sim 50 \mathrm{~min}$. This may explain the need for continuous delivery by osmotic minipumps. Pharmacokinetic calculations suggest that the serum level of continuously delivered anginex is approximately $2-3 \mu \mathrm{g} / \mathrm{ml}(1 \mu \mathrm{M})$. This is the concentration where we start seeing biological effects in in vitro models. The homing of anginex to tumor blood vessels may, however, lead to high drug levels in the local micro-environment of the tumor blood vessels. This may mean that the continuous maintenance of steady state plasma drug levels is unnecessary for the optimal efficacy of anginex.

Several lines of evidence are presented for the preferential activity of anginex on activated $\mathrm{EC}$. First, the results obtained from freshly isolated $\mathrm{EC}$ suggest that, although bFGF acted as a survival factor, anginex treatment in the presence of bFGF forced equivalent numbers of EC 
into apoptosis as compared to treatment with anginex in the absence of bFGF. This was a general observation in all EC cultures. Second, anginex binds more efficiently, the more an EC culture is activated. Third, in order to show loss of activity in quiescent EC, attempts were made to bring EC cultures in a lower metabolic status. This turned out to be difficult with starvation or inhibitors of protein/RNA synthesis. A double thymidine block, at least arresting the cells in early $S$ phase of cell cycle, resulted in loss of $\sim 50 \%$ of apoptosis induction by anginex. Fourth, fluorescently labeled anginex was found to specifically target tumor EC but not EC in normal tissues.

As for all angiogenesis inhibitors, the EC receptor that transduces the angiostatic signal is a relevant issue. ${ }^{140}$ The efficient migration inhibition by anginex suggests that the blockade of a matrix binding adhesion molecule may be important in its mechanism of action. Several of these molecules are up-regulated in tumor vessels. ${ }^{64,141}$ In co-localization studies, however, using antibodies against anginex and EC adhesion molecules, we were unable to identify either $\alpha_{v} \beta_{3}$ , $\alpha_{v} \beta_{5}-$, or $\beta_{1}$-integrins or CD44 as EC surface receptor for anginex. The generally experienced difficulty in identifying cell surface receptors for angiogenesis inhibitors, as well as the sparse reports such as those on ATP-synthase ${ }^{39}$, glypican ${ }^{142}$, and $\mathrm{CD} 36^{143}$ acting as receptors for angiostatin, endostatin, and thrombospondin-1, respectively, suggests that anti-angiogenic activities maybe mediated by a cell surface receptor-independent process or by indirect mechanisms such as induction of matrix instability.

The capacity of anginex to specifically home to tumor blood vessels might be used, on the one hand, to co-target isotopes, toxins or drugs. This feature of anginex is currently under investigation. On the other hand, the specific homing to angiogenically activated blood vessels may be an important tool for diagnostic use in the clinic to determine the amount of activated blood vessels in, or the angiogenic potential of tumors. The combination of targeting tumor blood vessels and having an angiostatic effect combined in one agent, may give anginex preferable features for tumor treatment. The current results indicate that anginex is a powerful anti-tumor angiogenesis inhibitor which shows promise and should be developed for the treatment of human cancers. Even though this paper reports only on the anti-tumor activity of anginex, the possible broader use of the peptide in the treatment of other diseases, such as rheumatoid arthritis, endometriosis, atherosclerosis, psoriasis, and ocular neovascularization, requires further investigation and is the subject of future research in our laboratories. 


\section{Chapter 4}

\section{The designed angiostatic peptide anginex synergistically improves chemotherapy and anti-angiogenesis therapy with angiostatin}

Ruud P.M. Dings, Yumi Yokoyama, S. Ramakrishnan, Arjan W. Griffioen, and Kevin H. Mayo.

Cancer Res. 2003; 63(2): 382-5. 


\section{Abstract}

Recently, we demonstrated that the designed peptide anginex displays potent antiangiogenic activity. The aim of the present study was to investigate anginex-treatment as a singleagent therapy and to test its ability to improve conventional chemo- and anti-angiogenesis therapy. In a human ovarian carcinoma mouse model, anginex inhibited tumor growth by $70 \%$. When anginex was combined with a sub-optimal dose of carboplatin, tumors regressed to an impalpable state. Anginex plus angiostatin worked synergistically to inhibit tumor growth. Assessment of microvessel density suggested that the anti-tumor activity of anginex is mediated by angiogenesis inhibition. In any of the experiments, no sign of anginex-induced toxicity was observed. 


\section{Introduction}

Neo-vascularization or angiogenesis is the process of new capillary outgrowth from preexisting blood vessels. Sustained angiogenesis is one of the essential alterations in cell physiology that collectively dictate malignant growth. ${ }^{144}$ Angiogenesis is required for solid tumors to grow beyond the size of approximately of $1-2 \mathrm{~mm}^{3}$. A highly vascularized tumor is associated with poor clinical prognosis, not only because of the potential for exponential tumor growth, but also because of the increased access capacity to the capillaries ${ }^{99}$, which supposedly facilitates metastasis formation ${ }^{100}$. Consequently, methods to inhibit angiogenic sprouting provide an unique opportunity to arrest tumor growth and prevent metastasis, either alone or in combination with conventional therapies. Combination of angiogenic inhibitors with radiation ${ }^{145,146}$, gene therapy ${ }^{147}$ or chemotherapy ${ }^{148}$ has been shown to be successful. Recently, we reported the design of anginex, a $\beta$-sheet forming peptide $33 \mathrm{mer}$, with potent in vitro anti-angiogenesis activity. ${ }^{36}$ The aims of the present study were to investigate anginex treatment in vivo as a single-agent therapy and in combination with conventional chemotherapy and the structurally unrelated anti-angiogenic angiostatin. Here, we show that although anginex alone significantly inhibits tumor growth, treatment in combination with a sub-optimal dose of carboplatin, results in tumor remissions to microscopic disease and in combination with angiostatin demonstrates a synergistic effect at inhibiting tumor growth. The present data warrant further development of anginex for clinical use.

\section{Materials and Methods}

\section{Reagents}

Anginex and control peptide, Bpep-28 were synthesized as described earlier. ${ }^{36}$ Bpep28 is $91 \%$ homologous and $67 \%$ identical peptide compared to anginex but has no anti-angiogenic activity (8). Carboplatin (Sigma Diagnostics; St Louis, MO) was dissolved in PBS (32.5 mg/kg) and administered once every three days i.p.. Angiostatin $(20 \mathrm{mg} / \mathrm{kg})$ was administered daily s.c. in the neck, as described previously. ${ }^{134}$

\section{Culture}

MA148, a human epithelial ovarian carcinoma cell line, was cultured on non-coated flasks using 10\% FBS, 1\% penicillin/streptomycin in RPMI 1640. Cultures were split 1:3 every 3 days. Mouse angiostatin (kringle 1-4) was cloned and expressed in Pichia pastoris ${ }^{149}$, with culturing, elution and purification being done as described previously. ${ }^{150,134}$ 


\section{Ovarian carcinoma mouse model}

Female athymic nude mice (nu/nu, 5-6 weeks old) were purchased from the National Cancer Institute and allowed to acclimatize for one week. Human ovarian MA148 epithelial carcinoma cells were cultured, harvested and inoculated subcutaneously into the right flank of the mouse as described previously. ${ }^{134}$ In the initial experiment, treatment was initiated after randomization of mice, and implanting osmotic mini-pumps (Durect; Cupertino, CA) into the left flank. The pumps had a treatment span of 28 days, which started on the same day as the inoculation of the ovarian carcinoma cells. Subsequently, studies were carried out in a therapeutic intervention model with established tumors to test the capacity of anginex to inhibit tumor growth and to test it in conjunction with angiostatin. In this latter model, treatment was initiated 7 days post-inoculation with the MA148 cells. To test the ability of anginex to enhance conventional chemotherapy, carboplatin was used in combination with anginex, in the same intervention model.

Tumor volume was determined by measuring the size of the tumors on the flanks of the mice. The diameters of tumors were measured using callipers (Scienceware; Pequannock, $\mathrm{NJ}$ ) and the volume was calculated using the equation to determine the volume of a spheroid: $\left(a^{2} \times b \times \Pi\right) / 6$, where ' $a$ ' is the width and ' $b$ ' is the length of the tumor.

\section{Immunohistochemistry}

Tumor tissues were embedded in tissue freezing medium (Miles Inc.; Elkart, IN) and snap frozen in liquid nitrogen. Preparation and procedures were done as described earlier. ${ }^{12}$ Samples were subsequently incubated in a 1:50 dilution with phyco-erytrin (PE)-conjugated monoclonal antibody to mouse CD31 (PECAM-1) (Pharmigen; San Diego, CA) or a fluorescien isothiocyanate (FITC)-conjugated PCNA (Ab-1) (Oncogene; San Diego, CA) to stain for microvessel density (MVD) or proliferation, respectively. After a 1-hour incubation at room temperature, slides were washed with PBS and immediately imaged in an Olympus BX-60 fluorescence microscope at 200X magnification. Sections were also stained for cell death using a TUNEL (terminal deoxyribonucleotidyl transferase-mediated dUTP-nick-end labelling) assay carried out according to the manufacturer's instructions (in situ cell death detection kit, fluorescein; TUNEL, Roche). Although the TUNEL assay detects apoptosis, it can not be ruled out that TUNEL will also stain for necrosis, where extensive DNA fragmentation may occur. Digital images were acquired and processed using Adobe Photoshop (Adobe Inc., Mountain View, CA). Vessel density was quantified as described earlier. ${ }^{112}$ Statistical analysis was performed using the Student's ttest.

\section{Toxicity assays}

As an indirect measurement of general toxicity, body weights of mice were monitored twice weekly, using a digital balance (Ohaus Florham, NJ). To determine hematocrit and creatinine levels, blood samples were extracted by tail vein bleedings one day after terminating treat- 
ment and blood was collected in heparinized micro-hematocrit capillary tubes (Fisher; Pittsburgh, PA). For hematocrit levels, samples were spun down for 10 minutes in a micro-hematocrit centrifuge (Clay-Adams; NY), and the amount of hematocrit was determined using an international microcapillary reader (IEC; Needham, Mass). To obtain creatinine levels, a kit was purchased from Sigma (Sigma Diagnostics; St Louis, MO) and used according to the manufacturer's instructions.

\section{Results}

\section{Anginex inhibits tumor growth in vivo}

Mice, inoculated with MA148 ovarian carcinoma cells, were randomized and treated systemically with anginex for 28 days using osmotic mini-pumps starting at the day of inoculation. A control peptide, $\beta$ pep- 28 , that is $91 \%$ sequentially homologous and $67 \%$ identical to anginex, was used to control for peptide content. Another set of animals was treated with vehicle containing BSA to control for protein content. As illustrated in Figure 1, treatment with anginex resulted in a dose-dependent inhibition of tumor growth that was maximal at $10 \mathrm{mg} / \mathrm{kg} /$ day as compared with vehicle-treated animals. At this dose, anginex inhibited about $70 \%$ of tumor growth. At half this dose, tumor growth was inhibited only by $50 \%$, whereas a higher dose $(20 \mathrm{mg} / \mathrm{kg} /$ day $)$ did not result in enhanced efficacy (Figure 1). Tumors from control peptide $\beta$ pep-28 treated mice did not differ in size from tumors in the BSA vehicle treated animals. Moreover, treatment with BSA or $\beta$ pep-28 did not result in altered tumor growth as compared to treatment with saline alone (data not shown).

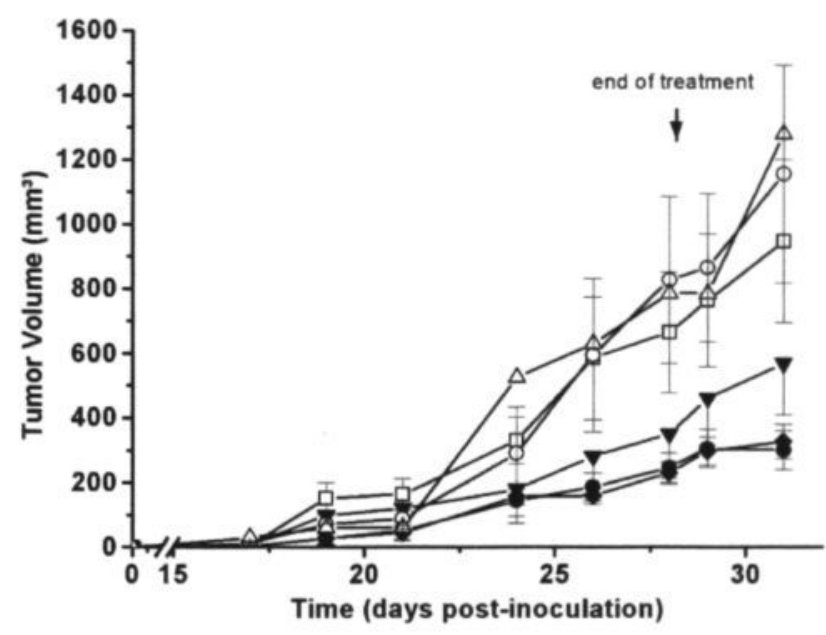

Figure 1. Anginex causes significant tumor growth inhibition. The mean tumor growth of human MA148 ovarian carcinoma is shown in athymic mice treated with a doserange of anginex \{ (closed triangle down) 5 $\mathrm{mg} / \mathrm{kg} / \mathrm{day}, n=14$; (closed circle) $10 \mathrm{mg} / \mathrm{kg} /$ day, $n=16$, or (closed diamond) $20 \mathrm{mg} / \mathrm{kg} /$ day, $n=8)$ \} administered by mini-pumps implanted in the left flank of animals. Controls (open square) contained PBS with BSA ( $n=$ 13 ) and PBS with Bpep- $285 \mathrm{mg} / \mathrm{kg} /$ day (open circle; $n=8$ ) and $10 \mathrm{mg} / \mathrm{kg} /$ day (open triangle up; $n=4$ ), which did not differ from each other. The treatment period was initiated on the day of tumor inoculation (day 0 ) and lasted for $\mathbf{2 8}$ days as indicated by the vertical arrow in the figure. Data from three independent studies are shown and represent the mean tumor volume in $\mathrm{mm}^{3}( \pm \mathrm{SE})$. 


\section{Anginex inhibits tumor growth of established tumors and improves conventional chemotherapy}

Since initial animal experiments were performed using an experimental set-up where treatment was started at the time of tumor inoculation (a system that models treatment of minimal residual disease), anginex was also tested using the MA148 model in an intervention set-up where treatment was initiated after tumor establishment. In these experiments, anginex inhibited tumor growth by approximately $50 \%$ (Figure $2 \mathrm{~B}$ ).

In an attempt to improve the efficacy of platinum-based chemotherapy, anginex was administered to tumor-bearing mice that were concurrently treated with a sub-optimal dose of carboplatin. Carboplatin treatment resulted in an effective reduction of tumor growth; however when it was combined with anginex, no tumor mass could be palpated in these mice (Figure 2A). One week after termination of treatment the tumor re-established.
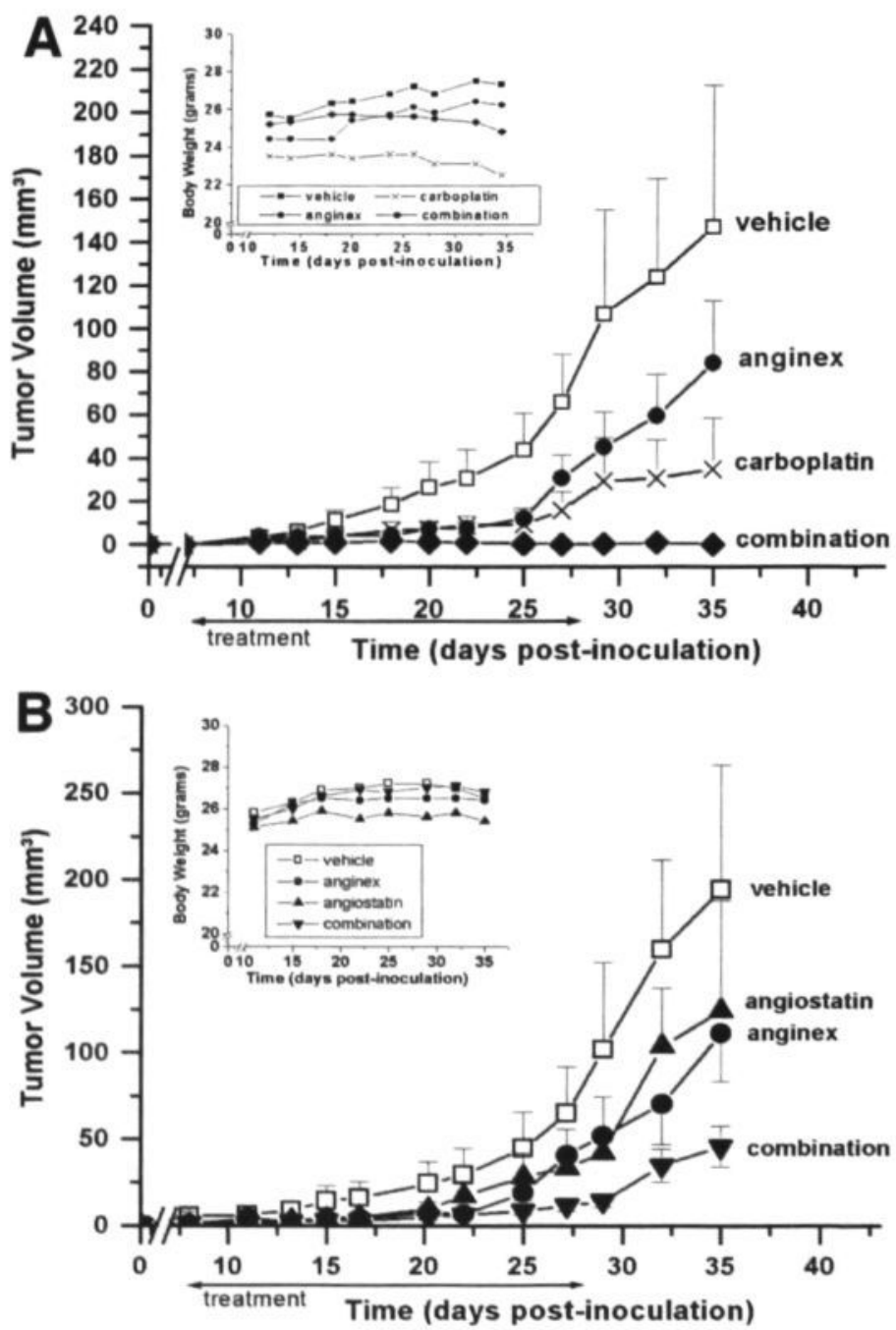

Figure 2. The mean tumor growth curves in a human ovarian carcinoma model treated with anginex, carboplatin, angiostatin or a combination treatment. In panel $2 \mathrm{~A}$ groups are shown defined as follows, vehicle containing BSA (open square, $n=$ 11 ), anginex (closed circle; $10 \mathrm{mg} / \mathrm{kg} /$ day, $n=11)$, carboplatin $(\mathrm{X} ; n=12)$ and a combination group (closed diamond; $n=$ 12). Carboplatin was given in a sub-optimal dosage $(32.5 \mathrm{mg} / \mathrm{kg})$ once every three days in an intra-peritoneal matter. In panel 2B groups are defined as vehicle containing BSA (open square, $n=11$ ), angiostatin (closed triangle up; $20 \mathrm{mg} / \mathrm{kg} /$ $\mathrm{d}, n=11$ ), anginex (closed circle; $10 \mathrm{mg}$ / $\mathrm{kg} /$ day, $n=11$ ) or combination (closed triangle down; $n=12$ ) of angiostatin and anginex in an ovarian carcinoma xenograft model. In both experiments treatment was given for 28 days starting on day 7 postinoculation. The vehicle and anginex were given by osmotic mini-pump implanted subcutaneously in the flank, and angiostatin was given daily by injections subcutaneously in the neck. ${ }^{134}$ The data are shown as means of tumor burden ( \pm $\mathrm{SE}$ ). The tumor volumes were determined three times a week. The insets in both panels show body weights of mice during treatment as an indirect measurement of toxicity. 


\section{Anginex and angiostatin act synergistically to inhibit tumor growth}

Using optimized treatment regimes for angiostatin ${ }^{134}$, and anginex, we found that both anginex and angiostatin, administered separately, inhibited tumor growth comparably by approximately $50 \%$, in the same ovarian tumor model. Based on those findings the interaction between angiostatin and anginex was examined. Combination therapy of angiostatin with anginex, resulted in enhanced tumor growth inhibition (80\%) (Figure $2 \mathrm{~B}$ ), which was deemed to be synergistic (Table 1).

Table 1. Combination therapy of anginex with carboplatin and angiostatin.

Fractional tumor volume (FTV) relative to untreated controls ${ }^{\text {a }}$

\begin{tabular}{|c|c|c|c|c|c|}
\hline & \multicolumn{4}{|c|}{$\begin{array}{l}\text { Combination } \\
\text { treatment }\end{array}$} & Ratio $^{\sigma}$ \\
\hline $\operatorname{Day}^{\circ}$ & Anginex & Carboplatin & Expected $^{c}$ & Observed & $\begin{array}{l}\text { Expected FTV/ } \\
\text { Observed FTV }\end{array}$ \\
\hline 20 & 0.28 & 0.28 & 0.08 & 0.05 & 1.6 \\
\hline 25 & 0.28 & 0.22 & 0.06 & 0.01 & 6 \\
\hline 32 & 0.48 & 0.25 & 0.12 & 0.01 & 12.4 \\
\hline \multirow[t]{2}{*}{35} & 0.57 & 0.24 & 0.13 & 0 & $\infty$ \\
\hline & \multicolumn{4}{|c|}{$\begin{array}{l}\text { Combination } \\
\text { treatment }\end{array}$} & Ratio $^{\sigma}$ \\
\hline Day $^{b}$ & Anginex & Angiostatin & Expected $^{c}$ & Observed & $\begin{array}{l}\text { Expected FTV/ } \\
\text { Observed FTV }\end{array}$ \\
\hline 25 & 0.42 & 0.64 & 0.27 & 0.19 & 1.4 \\
\hline 29 & 0.51 & 0.41 & 0.21 & 0.14 & 1.5 \\
\hline 32 & 0.44 & 0.65 & 0.29 & 0.22 & 1.3 \\
\hline 35 & 0.57 & 0.64 & 0.37 & 0.24 & 1.6 \\
\hline
\end{tabular}

${ }^{a}$ FTV (mean tumor volume experimental)/(mean tumor volume control).

${ }^{b}$ Day after tumor cell transplantation.

${ }^{c}$ (Mean FTV of anginex) X (mean FTV of other experimental group).

d Obtained by dividing the expected FTV by the observed FTV. A ratio of $>1$ indicates a synergistic effect, a ratio of $<1$ indicates a less then additive effect.

\section{Toxicity}

Animals treated with anginex (alone or in combination regimens) did not show any sign of toxicity as assessed by unaltered behaviour, weight gain during experiments, normal hematocrit and creatinine levels, and macro- and microscopic morphology of internal organs on autopsy. Body weights of mice were monitored as an indirect measurement of general toxicity. In experiments where carboplatin was administered, the weights of mice actually fell initially and subsequently increased on termination of exposure to carboplatin. This was taken as a sign of mild reversible toxicity. Anginex did not augment this toxicity. One day after the termination of treatment, blood was drawn and hematocrit and creatinine levels were determined as a measure of bone marrow and kidney toxicity, respectively. Hematocrit levels reported as a percentage of red blood cells (vehicle $50.2 \pm 2.9$, anginex $51.3 \pm 2.5$, carboplatin $49.3 \pm 2.8$, and combination 
$47.2 \pm 2.4$ ) and creatinine levels reported in $\mu$ moles/l (vehicle $46.8 \pm 8$, anginex $48 \pm 1.4$, carboplatin $55.5 \pm 12.6$, and combination $42 \pm 5.3$ ) showed no significant difference in the study involving carboplatin. The study combining anginex and angiostatin treatment showed similar hematocrit levels (vehicle $49 \pm 1.7$, anginex $49.2 \pm 2.6$, angiostatin $47.8 \pm 2.1$, and combination $48.3 \pm 1.9$ in percentage red blood cells) and creatinine levels (vehicle $46.8 \pm 6.4$, anginex $48 \pm$ 1.4 , angiostatin $41 \pm 0.4$, and combination $39.2 \pm 5.9$ all in $\mu$ moles/l).

A

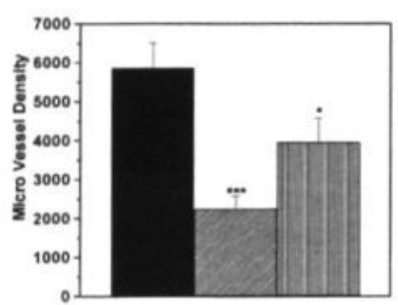

C
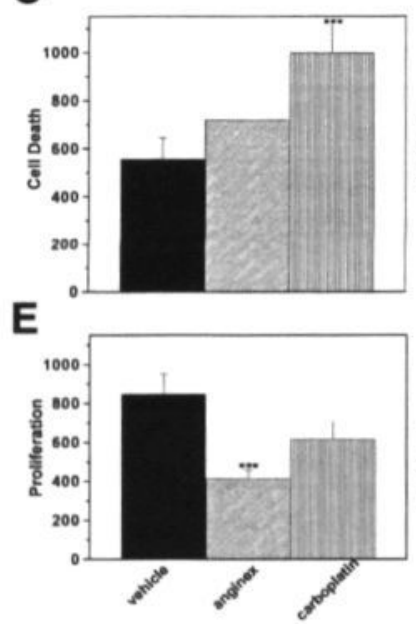
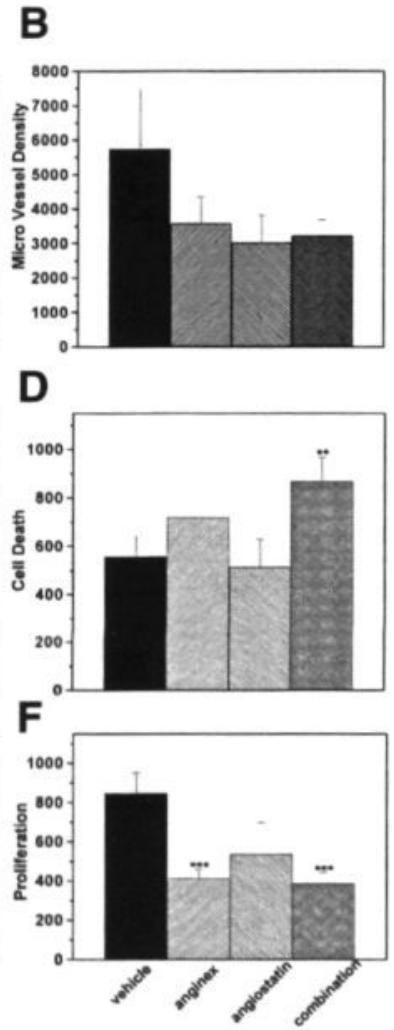

Figure 3. Immunohistochemistry analyses. After snap freezing tumor tissues, $10 \mu \mathrm{m}$ sections were made and stained for microvessel density by using anti-CD31 antibodies (panel A and B), cell death by TUNEL (panel C and D) and proliferation by PCNA (panel $\mathrm{E}$ and F), all expressed in number of white pixels. The procedure and quantification were described earlier. ${ }^{112}$ Panel A, C, and $\mathrm{E}$ show quantifications of the studies involving anginex and carboplatin. Panels B, D and F show quantification of studies involving anginex, angiostatin or combination therapy. As determined by using the Student's t-test relative to the vehicle group, ${ }^{*} P<0.05,{ }^{* *} P<0.03$, $* * * P<0.01$.

\section{Histological analysis of microvessel density, cell death and proliferation}

Anginex treatment resulted in a decrease of tumor microvessel density (MVD), suggesting that the anti-tumor activity of anginex is the result of angiogenesis inhibition. Angiostatin demonstrated a similar result. Although anginex and angiostatin acted synergistically on tumor growth inhibition, this was not reflected in the MVD assessment (Figure 3B). Aside from vessel density (including number, size and length) ${ }^{112}$, the digital approach discriminates branch points, end points and vessel lengths. Some of these architectural parameters did change upon combination treatment relative to single agent treatment. For example, combination treatment revealed a synergistic reduction in the number of branch points (data not shown). Tumors from anginex-treated animals showed a convincing MVD reduction $(P<0.01)$, whereas tumors from carboplatin-treated animals showed a smaller, albeit significant, reduction in MVD $(P<0.05$; 
Figure $3 \mathrm{~A})$. Tumors treated with the combination of anginex and carboplatin could not be stained because all tumors disappeared three weeks after initiation of treatment.

Although combination of anginex and angiostatin also showed a synergistic effect in the increased amount of cell death $(P<0.03)$, as determined by TUNEL analysis, angiostatin by itself did not trigger increased cell death compared to the vehicle group (Figure 3D). As expected, carboplatin did show an increase in the amount of cells undergoing cell death $(P<0.01)$. A down regulation in proliferation, as determined by PCNA staining, was revealed in all treated groups compared to controls, significant for anginex and combination treated groups $(P<0.01$; Figures $3 E$ and $F)$.

\section{Discussion}

Induction of angiogenesis by malignant cells has been shown to play a pivotal role in the process of tumor proliferation and metastasis. ${ }^{15}$ Inhibition of angiogenesis is, therefore, a promising way to arrest tumor growth and prevent metastasis. Due to the need for new antitumor agents with improved potency, stability, selectivity and ease of delivery, we used a novel approach in designing the $\beta$-sheet-forming anginex peptide. ${ }^{36}$ Anginex acts specifically on activated endothelial cells (EC) to trigger apoptosis, presumably by preventing cell adhesion and subsequent induction of anoikis. ${ }^{36} \mathrm{Here}$, we demonstrate that anginex is an anti-angiogenic compound with anti-tumor activity when administered systemically as a single-agent therapeutic.

Because anti-angiogenic agents can potentiate cytotoxic cancer therapies ${ }^{148}$, anginex was tested in combination with the chemotherapeutic carboplatin. Platinum agents are the most widely used drugs in the first-line of defense against ovarian cancer. ${ }^{151,152}$ In a recent study, singleagent carboplatin proved to be just as effective as carboplatin plus paclitaxel in women requiring chemotherapy for ovarian cancer. The favorable toxicity profile of carboplatin alone suggested that this is a reasonable option as a single-agent chemotherapeutic. ${ }^{153} \mathrm{An}$ additional advantage of carboplatin is that, in contrast to other agents such as taxanes, cyclophosphamide and vincristine, it is not an anti-angiogenic by itself. Because carboplatin has been shown to be a very powerful anti-cancer drug in the model used here, a sub-optimal dose was employed. Both anginex and carboplatin inhibited tumor growth, but the combination blocked tumor growth completely, and palpable tumors regressed to undetectable sizes in all animals. Moreover, tumors remained undetectable until at least one week after termination of the treatment, after which tumors re-established themselves, indicating continued presence of microscopic disease, which was unresponsive to carboplatin and apparently independent of angiogenesis.

Whereas numerous investigators have focused on the anti-cancer effects from endostatin in their tumor model studies, we chose to use angiostatin because in the same MA148 tumor mouse model, Yokoyama et al. ${ }^{134}$ found that angiostatin was considerably more effective at 
inhibiting tumor growth than endostatin. Here, we found that although treatment with angiostatin or anginex showed essentially the same capacity to inhibit tumor growth, combination of the two produced a dramatically enhanced inhibitory effect. This synergy between angiostatin and anginex suggests that their mechanisms of action are different and that they can augment each other as anti-angiogenic agents. These data, therefore, provide a validation for combination therapy, if not for cocktails of angiogenesis inhibitors, to improve the treatment of cancer.

Immunohistochemical assessment of MVD indicated that tumor growth inhibition by anginex is explained by its anti-angiogenic activity. Angiostatin showed the same trend. Combination therapy, however, did not yield an increased MVD reduction. This may be explained by the fact that differences in architecture are not represented in the MVD value assessed either digitally or manually. The digital approach discriminates for architectural parameters, some of which did change upon combination treatment relative to single agent treatment. For example, combination treatment revealed a synergistic reduction in the number of branch points (data not shown).

This study adds credence to the proposal that cancer treatment using anti-angiogenesis agents is more effective when performed in combination with other agents. However, it might be that only cocktails of anti-angiogenic compounds would provide sufficient potency to be evaluated properly in early clinical trials. Currently, clinical evaluation of anti-angiogenic compounds is in its infancy, and while a stand-alone approach using an anti-angiogenic agent shows promise, combination therapy may provide for the best evaluation of these agents and, in the end, may be more beneficial. Present results suggest that combination of anti-angiogenics with chemotherapeutics will produce a greater effect than combinations of anti-angiogenic agents alone. At the very least, this study indicates that combining the anti-angiogenic compound anginex with conventional chemotherapy allows dosage of the chemotherapeutic to be reduced, while still being able to effect tumor growth reduction. 


\section{Chapter 5}

\section{B-Sheet is the bioactive conformation of the anti-angiogenic anginex peptide}

Ruud P.M. Dings, Monica M. Arroyo, Nathan A. Lockwood, Loes I. van Eijk, Judy R. Haseman, Arjan W. Griffioen, and Kevin H. Mayo

Biochem J. 2003; 373: 1-6. 


\section{Abstract}

Anginex is a designed peptide 33 mer that functions as a cytokine-like agent to inhibit angiogenesis. Although this short linear peptide has been shown by NMR and CD to form nascent B-sheet conformation in solution, the actual bioactive structure formed upon binding its receptor on the surface of endothelial cells could be quite different. Here, we first show that a shorter sequence of anginex, missing its six $\mathrm{C}$-terminal residues, is equipotent with parent anginex. To test the hypothesis that a $\beta$-sheet defines the bio-active conformation of anginex, a series of double cysteine-containing analogs of the linear $27 \mathrm{mer}$ was synthesized and oxidized to form disulfide bonds between peptide segments as in a B-sheet. These covalent constraints limited conformational space available to the peptide, such that, for example, helix formation could not occur. CD spectral analysis of the linear peptide 27 mer and all disulfide-linked analogs indicated formation of $\beta$-sheet conformation. 'H NMR studies demonstrated that most of these, however, do not form well-folded $B$-sheets and exist in a rapidly exchanging distribution of conformations. Nevertheless, $\alpha \mathrm{H}-\mathrm{NH}$ resonance chemical shifts are shifted downfield, consistent with significant populations of $B$-sheet. One disulfide-linked analog, C6-C25 (CP-2), which showed welldispersed ${ }^{1} \mathrm{H}$ NMR signals, allowed NOE-derived structures to be determined. Disulfide-bridged analogs were analyzed in three functional assays: endothelial cell proliferation, apoptosis, and in vitro angiogenesis. Results from all these assays were comparable in that as long as placement of disulfide bonds preserved the B-strand alignment as in the proposed bioactive conformation, activity was preserved. Subtle differences in bioactivities among disulfide-linked analogs are discussed in terms of structure and placement of disulfide bonds. 


\section{Introduction}

Angiogenesis, the formation of new blood vessels out of pre-existing capillaries, is pivotal to a broad array of biological functions ranging from normal processes like embryogenesis and wound healing to abnormal processes like tumor growth, arthritis, restenosis and diabetic retinopathy. ${ }^{10,96}$ The use of agents that can inhibit angiogenesis in vitro and in vivo, particularly in anti-tumor research, has indicated that anti-angiogenic therapy can be a therapeutic modality in the future. Most anti-angiogenic agents have been discovered by identifying endogenous molecules, primarily proteins, which inhibit EC growth. This traditional approach has produced a number of anti-angiogenics, such as platelet factor- 4 (PF4) ${ }^{103}$, thrombospondin- $1^{101}$, interferon- $\alpha$ inducible protein-10 ${ }^{125}$, angiostatin ${ }^{8}$, endostatin ${ }^{9}$, and bactericidal-permeability increasing (BPI) protein $^{82}$.

Recently, we reported the anti-angiogenic activity of a novel peptide 33mer (Bpep-25 or anginex). ${ }^{36,95}$ Anginex belongs to a family of homologous Bpep peptides that were designed by using basic folding principles and incorporating short sequences from the $\beta$-sheet domains of $\alpha$ chemokines and BPI protein. ${ }^{77}$ All Bpep peptides form B-sheets, to varying degrees, and can self-associate in solution as dimers and tetramers. ${ }^{77,95} \mathrm{~A}$ high resolution NMR structure of tetrameric Bpep-4 demonstrated that its monomer subunits are folded into an amphipathic, threestranded anti-parallel ß-sheet motif. ${ }^{78}$ Two types of six-stranded anti-parallel ß-sheet dimers can form, and these dimers associate via their hydrophobic faces into tetramers. Although a high resolution structure of anginex (Bpep-25) could not be determined by using NMR because of aggregate exchange resonance broadening, circular dichroism and NMR studies indicate that anginex also folds primarily into tetrameric $B$-sheets at millimolar concentration. At concentrations in the micromolar range and lower, however, anginex exists in solution primarily in the monomer state that is composed of a relatively broad distribution of conformations with considerable random coil and some $B$-sheet character. ${ }^{94}$ Therefore, at the micromolar concentrations used in in vitro bioassays and in vivo animal studies demonstrating the anti-angiogenic ${ }^{95,36}$ and antitumor ${ }^{154-156}$ potency of the peptide, anginex is mostly random coil monomer. The bioactive structure of anginex, which is selected from this rather broad conformational distribution, therefore, remains unclear.

The main goal of structural biologists and pharmaceutical chemists working to develop better and more effective anti-angiogenic agents and their small molecule mimetics is the identification of specific amino acid residues and their spatial relationships that are key to promoting anti-angiogenic effects at the level of molecular interactions. Knowing the bioactive conformation, therefore, is crucial to this endeavor. One way to assess this in a small peptide like anginex is to limit the conformational space available to its amino acid sequence by placing covalent bonds between specific residues. The present study was aimed at testing the hypothesis that the bioactive conformation of anginex (Bpep-25) is B-sheet with the same strand alignment as in the 
NMR-derived structure of homologous Bpep- $4 .^{78}$ To accomplish this, a series of disulfide-linked peptide analogs of anginex was made (see Figure 1). By comparing activities from all $30 \mathrm{mem}-$ bers of the Bpep library, it was already deduced ${ }^{95}$ that functionally key residues in anginex are primarily hydrophobic residues, i.e., L5, V7, I20, V22 and L24. Because of this, cysteines replaced residues primarily at positions that would be on the hydrophilic face of the amphipathic anginex $B$-sheet. Moreover, to simplify peptide synthesis and purification, six functionally inessential residues at the $\mathrm{C}$-terminus of anginex ${ }^{95}$ were removed, creating a peptide 27 mer analog of anginex that is shown here to be equipotent to the parent peptide. Double-substituted cysteine pairs were made: $\mathrm{C} 6-\mathrm{C} 25, \mathrm{C} 8-\mathrm{C} 23, \mathrm{C} 10-\mathrm{C} 21, \mathrm{C} 12-\mathrm{C} 19, \mathrm{C} 13-\mathrm{C} 18$, and control peptides C13-C19 and C6-C26. These disulfide-bridged analogs were analyzed in three functional assays: endothelial cell proliferation, apoptosis, collagen gel sprouting. Peptide conformation was assessed with circular dichroism (CD) and ${ }^{1} \mathrm{H}$ NMR spectroscopies.

\section{Materials and Methods}

\section{Peptide preperation}

Peptides were synthesized using a Milligen/Biosearch 9600 peptide solid-phase synthesizer using fluorenylmethoxycarbonyl chemistry. Peptide was cleaved from the resin, deprotected and then lyophilized. To form intramolecular disulfide bonds, crude peptides were solubilized to low concentration ( 100 micromolar), and cysteines were oxidized by slowly bubbling wet oxygen gas into a stirred aqueous solution overnight at room temperature. Lyophilized crude peptides (oxidized) were purified by preparative reversed-phase HPLC on a C18 column with an elution gradient of $0-60 \%$ acetonitrile with $0.1 \%$ trifluoroacetic acid in water. Purity and composition of the peptides were verified by HPLC (Beckman Model 6300), amino acid analysis and mass spectrometry. The presence of the disulfide bond was checked in all peptides by using the DTNB (Ellman's) reagent pre- and post-treatment with DTT.

\section{Circular dichroism}

Aqueous solutions for $\mathrm{CD}$ were prepared by dissolving lyophilized peptide in the appropriate amount of water to give final peptide concentrations of $0.1 \mathrm{mM}, \mathrm{pH} 5.5$. Concentrations were verified by tryptophan absorption at $280 \mathrm{~nm}$. CD spectra were recorded on a Jasco J-710 spectrophotometer using either a 0.01 or $0.1 \mathrm{~cm}$ path-length, thermally-jacketed quartz cuvette maintained at 24 or $37^{\circ} \mathrm{C}$ with a NesLab water bath. Acquisition was performed using a $50 \mathrm{~nm} / \mathrm{min}$ scan rate, $1 \mathrm{~nm}$ bandwidth, and $2 \mathrm{~s}$ response. The appropriate baseline was subtracted from each spectrum. Reported spectra are averages of six scans and are expressed as mean residue ellipticity, $[\theta]$. CD basis spectra ( $\alpha$-helix, $\beta$-sheet and random coil) were measured with poly(lysine) and poly(glutamic acid) (Sigma) using conditions and parameters reported by oth- 
ers. ${ }^{157,158}$ Experimental CD spectra were fit with a linear combination of $\alpha$-helix, $\beta$-sheet and random coil basis spectra to estimate secondary structure contributions.

\section{NMR measurements}

For NMR measurements, freeze-dried peptide was dissolved in $\mathrm{H}_{2} \mathrm{O}$ at a concentration of approximately $2 \mathrm{mM}$. $\mathrm{pH}$ was adjusted to $\mathrm{pH} 5.5$ by adding $\mu$ quantities of $\mathrm{NaOH}$ or $\mathrm{HCl}$ to the peptide sample. NMR spectra were acquired on a Varian UNITY Plus-600 NMR spectrometer. Water resonance was suppressed by direct irradiation $(0.8 \mathrm{~s})$ at the water frequency during the relaxation delay between scans.

2D-homonuclear magnetization transfer (HOHAHA) spectra, obtained by spin-locking with a MLEV-17 sequence ${ }^{159}$ with a mixing time of $60 \mathrm{~ms}$, were used to identify spin systems. NOESY experiments ${ }^{160}$ were performed for conformational analysis. All 2D-NMR spectra were acquired in the States-TPPI phase sensitive mode. ${ }^{161,162}$ The water resonance was suppressed by direct irradiation $(0.8 \mathrm{~s})$ at the water frequency during the relaxation delay between scans as well as during the mixing time in NOESY experiments. 2D-NMR spectra were collected as 256 to $512 \mathrm{t} 1$ experiments, each with $2 \mathrm{k}$ complex data points over a spectral width of $3 \mathrm{kHz}$ in both dimensions with the carrier placed on the water resonance. For HOHAHA and NOESY spectra, 32 scans were time averaged per $\mathrm{t} 1$ experiment. Data were processed directly on the spectrometer or offline using VNMR (Varian, Inc., Palo Alto) or NMRPipe ${ }^{163}$ on an SGI workstation. Data sets were multiplied in both dimensions by a 30 to 60 degree shifted sine-bell function and zero-filled to $2 \mathrm{k}$ in the $\mathrm{t} 1 \mathrm{dimension}$ prior to Fourier transformation. Data were analyzed using Sparky (T. D. Goddard and D. G. Kneller, SPARKY 3, University of California, San Francisco).

Since CP peptides are relatively hydrophobic and amphipathic, pulsed field gradient (PFG) NMR self-diffusion measurements were performed to investigate possible peptide aggregation. PFG-NMR experiments were done as described by Mayo et al. ${ }^{77}$ using a Varian Unity-Plus 500 NMR spectrometer. The maximum magnitude of the gradient was $60 \mathrm{G} / \mathrm{cm}$, and the PFG longitudinal eddy-current delay pulse-sequence was used for all self-diffusion measurements which were performed in $\mathrm{D}_{2} \mathrm{O}$ at temperatures of $5^{\circ} \mathrm{C}$ and $40^{\circ} \mathrm{C}$. Peptide concentrations ranged from $0.1 \mathrm{mM}$ to $2 \mathrm{mM}$.

\section{Structural modeling}

Analysis of NOE growth curves indicated that backbone to backbone inter-proton NOEs were normally maximum at $300 \mathrm{~ms}$ to $400 \mathrm{~ms}$. Interproton distance constraints were derived from NOEs assigned in $1 \mathrm{H}$ NOESY spectra acquired with a mixing time of $200 \mathrm{~ms}$. NOEs were classified as strong, medium, weak or very weak corresponding to upper bound distance constraints of $2.8,3.3,4.0$, and $4.5 \AA$, respectively. The lower bound restraint between non-bonded protons was set to $1.8 \AA$. Pseudo-atom corrections were added to the upper bound distance constraints where appropriate, and a $0.5 \AA$ correction was added to the upper bound for NOEs 
involving methyl protons. Hydrogen bond constraints were identified from the pattern of sequential and interstrand NOEs involving $\mathrm{NH}$ and $\mathrm{C} \alpha \mathrm{H}$ protons, together with evidence of slow amide proton-solvent exchange. Each hydrogen bond identified was defined using two distance constraints; $r_{\mathrm{NH}-0}=1.8$ to $2.5 \AA$, and $r_{n-0}=1.8$ to $2.5 \AA$.

Derived internuclear distance constraints were used in calculating structures for CP2 by using X-PLOR. ${ }^{164} \mathrm{CP} 2$ was created using parallhdg.pro force fields. A template coordinate set was generated by using the Template routine. The $a b$ initio simulated annealing (SA) protocol was then used. The SA procedure ran high temperature dynamics ( $3000 \mathrm{~K}$ for $120 \mathrm{ps})$ and then cooled down to $100 \mathrm{~K}$ in $50 \mathrm{~K}$ steps with $1.5 \mathrm{ps}$ molecular dynamics at each step. Powell minimization was performed at $100 \mathrm{~K}$ for 1000 steps. Structure refinement was done based on simulated annealing starting at $1000 \mathrm{~K}$ and ending at $100 \mathrm{~K}$. Final structures were subjected to the $\mathrm{X}$ PLOR Accept routine with the violation threshold for NOEs of $0.5 \AA$ and dihedral angles of $5^{\circ}$. Angles, bond lengths or impropers were not allowed to deviate from ideal geometry more than $5^{\circ}, 0.05 \AA$ and $5^{\circ}$, respectively. Structures were superimposed using the SwissPdbViewer and were analyzed using X-PLOR analysis routines.

\section{HUVEC cultures}

Human umbilical vein derived EC (HUVEC) were harvested from normal human umbilical cords by perfusion with $0.125 \%$ trypsin/EDTA. Harvested HUVECs were cultured in gelatin coated tissue culture flasks and subcultured 1: 3 once a week in culture medium (RPMI 1640 with $20 \%$ human serum (HS), supplemented with $2 \mathrm{mM}$ glutamine and $100 \mathrm{U} / \mathrm{ml}$ penicillin and $0.1 \mathrm{mg} / \mathrm{ml}$ streptomycin).

\section{Proliferation measurement}

EC proliferation was measured using a $\left[{ }^{3} \mathrm{H}\right]$-thymidine incorporation assay. EC were seeded at 5000 cells/well in flatbottomed tissue culture plates and grown for 3 days, in the absence or presence of regulators, in culture medium. During the last 6 hours of the assay, the culture was pulsed with $0.5 \mu \mathrm{Ci}\left[\right.$ methyl- ${ }^{3} \mathrm{H}$ ] thymidine/well. Results are expressed as the arithmetic mean counts per minute (cpm) of triplicate cultures.

\section{Apoptosis measurement}

HUVEC were cultured in fibronectin coated tissue culture flasks in culture medium. ${ }^{165}$ Apoptosis was measured by determination of subdiploid cells after DNA extraction and subsequent staining with propidium iodide as described before. ${ }^{166}$ In short, HUVEC were cultured for 3 days in the presence of $10 \mathrm{ng} / \mathrm{ml}$ bFGF and conditions as mentioned above. Serum deprivation of $\mathrm{EC}$ was used as a positive control for apoptosis. Cells were then harvested and subsequently fixed and permeabilized in $70 \%$ ethanol at $-20^{\circ} \mathrm{C}$. After at least 2 hours the cells were spun down and resuspended in DNA extraction buffer $\left(45 \mathrm{mM} \mathrm{Na}_{2} \mathrm{HPO}_{4}, 2.5 \mathrm{mM}\right.$ citric acid 
and $0.1 \%$ Triton $\mathrm{X}-100$ ) for 20 minutes. Propidium iodide (PI) was added to a final concentration of $20 \mathrm{mg} / \mathrm{ml}$ and red log-scale fluorescence was analyzed on the FACS-calibur. Apoptosis was quantified as the percentage of cells with $\mathrm{PI}$ fluorescence below the fluorescence of cells in $\mathrm{G}_{0} / \mathrm{G}_{1}$ (diploid) phase of cell cycle. Cells with PI fluorescence less than $10 \%$ of cells in $\mathrm{G}_{0} / \mathrm{G}_{1}$ phase were regarded as cells in advanced stages of cell death.

\section{In vitro angiogenesis assay}

Sprouting and tube formation of bovine EC (BCE) were studied using cytodex-3 beads overgrown with BCE in a 3-dimensional collagen gel (vitrogen-100, Collagen Corp., Fremont, CA, USA) as described by van der Schaft et al.. ${ }^{132}$ Following gelation, culture medium containing $20 \mathrm{ng} / \mathrm{ml} \mathrm{bFGF}$, with or without anginex or CP analogs, was applied on top of the gel. Photographs were made after 24 hours of cell culture at $37^{\circ} \mathrm{C}$. The amount of sprouting in each well (i.e. the total length of the sprouts) was quantified by the computer program $\mathrm{NIH}$ image. ${ }^{167}$ To quantify differences in sprouting and tube formation, statistical analysis was performed using the MannWhitney U test.

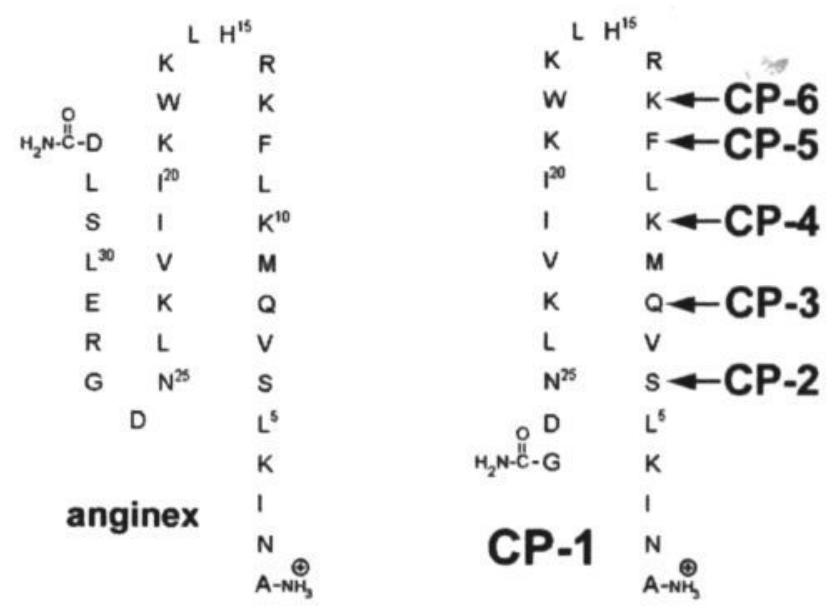

Figure 1. Amino acid sequence and structure of anginex and CP peptides. The amino acid sequence of anginex is shown conformed as an anti-parallel B-sheet. The $\mathrm{N}$-terminus is at the right bottom as labelled. CP peptides have only the first 27 residues, and therefore are devoid of C-terminal residues ...RELSLD. The new C-terminal residue $\mathrm{G}$ has its backbone carboxylate group amidated. CP-1 is the linear peptide $27 \mathrm{mer}$. CP-2 through CP-6 have disulfide bridges positioned between $B$-strand 1 and $B$-strand 2 in the sequence as indicated with arrows in the figure.

\section{Results}

For the cyclized peptide (CP) series, all CP peptides have the same sequence as anginex minus the six C-terminal residues RELSLD (Figure 1). CP-1 is the control linear peptide 27 mer. Amino acid sequences for all other CP peptides, showing the positions of disulfide bonds, are displayed in Figure 1. Because functionally key residues in anginex are primarily hydrophobic, i.e., L5, V7, 120, V22 and L24, and would lie on one side of the amphipathic structure in a B-sheet conformation ${ }^{95}$, residues on the hydrophilic face (and not the hydrophobic face) of the proposed B-sheet were substituted with cysteines. For assurance that cysteine-substituted positions did 
not affect activity of the parent peptide, single-substituted alanine variants were also made and tested in the in vitro EC proliferation assay. Relative to the parent peptide, activities were unaffected solely by amino acid substitutions at these positions (data not shown). On forming an actual B-sheet in CP-2 through CP-6, positioning of disulfide bonds constrains the B-strand alignment to be the same as that proposed for the folding of anginex. ${ }^{36} \mathrm{CP}-7$ [K13C; K19C] and CP-8 [S6C; D26C] are control peptides that shift the ß-strand alignment by one residue to be out of register from the other $\mathrm{CP}$ peptides.

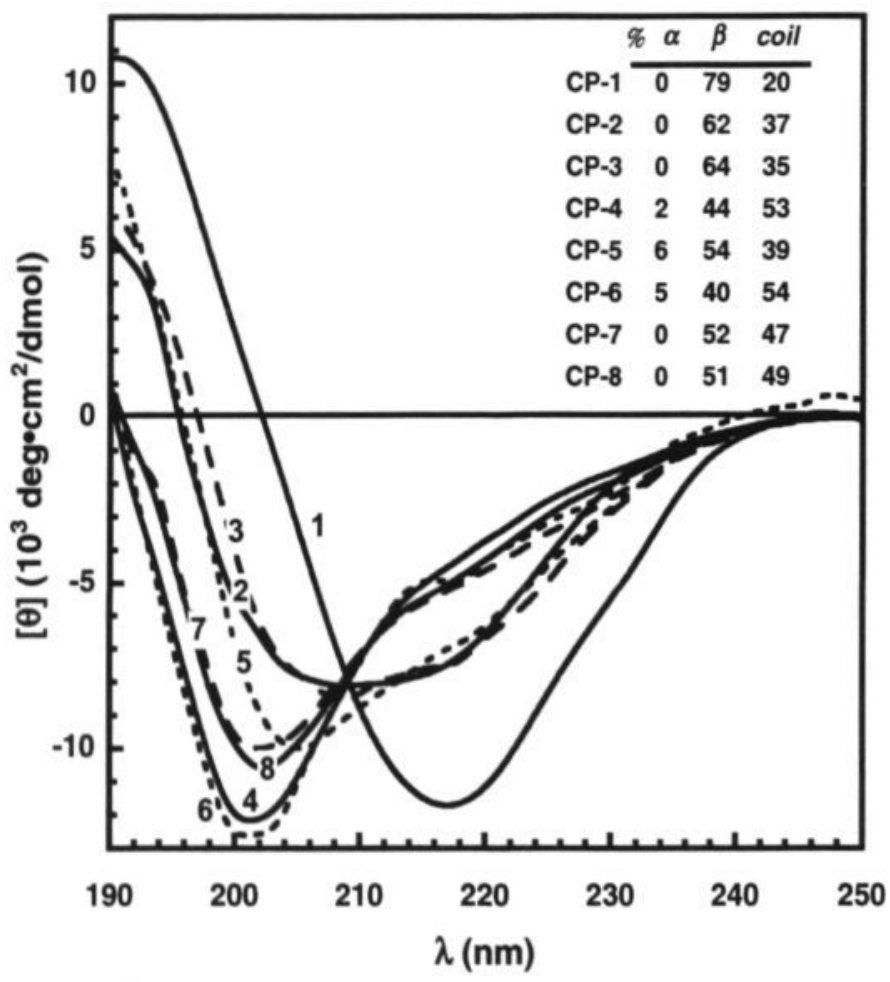

Figure 2. CD spectra for CP peptides. Far-ultraviolet circular dichroic spectra for CP peptides are shown as mean residue ellipticity, $[\theta]$, versus wavelength. All spectra display a mixture of B-sheet and random coil conformation, as shown by linear fits of basis spectra (inset). Peptide concentration was $0.1 \mathrm{mM}$ in water, $\mathrm{pH} 5.5$. Spectra shown were obtained at $37^{\circ} \mathrm{C}$; spectra at $24^{\circ} \mathrm{C}$ were comparable. Other experimental conditions are discussed in the Methods Section.

\section{Circular Dichroism}

CD and NMR spectroscopies were used to assess the conformation of CP peptides. For all CP peptides, far-UV CD traces indicated a mixture of $\beta$-sheet and random coil conformations (Figure 2 ), with the degree of $\beta$-sheet character corresponding to the intensity of the characteristic $217 \mathrm{~nm}$ trough ${ }^{158,168,169}$. Linear combinations of secondary structure basis spectra ( $\alpha$-helix, $\beta$-sheet, random coil) fit to the CP peptide spectra (Figure 2, inset) showed the largest B-sheet content $(79 \%)$ in the linear CP-1 peptide and the smallest ( $40 \%)$ in CP-6. Fits indicate the absence of helical content for most of the CP pepitdes. Even though CP-4, CP-5, and CP-6 show $2 \%$ to $6 \%$ helix content, this contribution is small enough that it is within the error from the fitting process. In addition, because the $\mathrm{CP}$ peptides are relatively flexible, the secondary structure contributions are only meant to semi-quantify the trend in $\beta$-sheet content. Nevertheless, these $\mathrm{CP}$ peptide conformational distributions are consistent with that for anginex. ${ }^{78}$ 


\section{'H NMR conformational analysis}

Even though $C D$ data demonstrate the presence of significant populations of $B$-sheet in all CP peptides, proton NMR spectra for all CP peptides, except CP-2, are characteristic of mostly random coil conformation (data not shown). CP-2 is the only one that shows well-dispersed resonances indicative of well-folded structure. ${ }^{170,171}$ Since parent anginex exists in solution in a monomer-dimer-tetramer equilibrium ${ }^{162,171}$, pulsed field gradient (PFG) NMR self-diffusion measurements were performed on CP peptides (data not shown). Analysis of the CP peptide diffusivities indicates that at $2 \mathrm{mM}$ peptide concentration, CP-2 is mostly dimeric, whereas the other CP peptides are a mixture of dimers and monomers. None of the CP peptides apparently associates to the tetramer state like anginex.

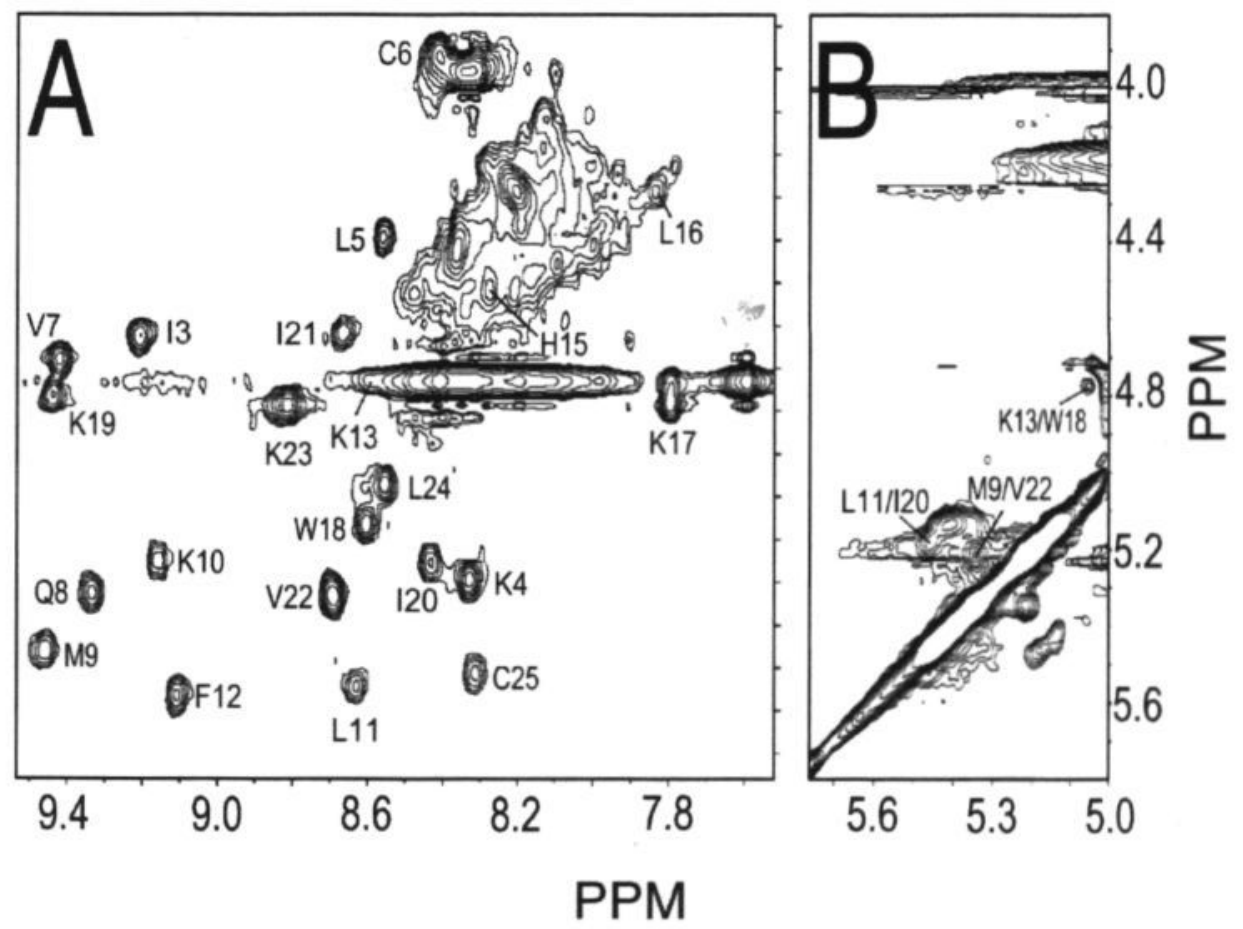

Figure 3. TOCSY and NOESY spectra for CP-2. $600 \mathrm{MHz}{ }^{1} \mathrm{H}$ TOCSY (A) and NOESY (B) spectra are shown for CP-2. Peptide concentration was $2.3 \mathrm{mM}$ in $\mathrm{H}_{2} \mathrm{O}_{2} \mathrm{D}_{2} \mathrm{O}(90 / 10)$, pH 5.5 and $40^{\circ} \mathrm{C}$. Spectra were accumulated with $8 \mathrm{k}$ data points over $8000 \mathrm{~Hz}$ sweep width and were processed with $1 \mathrm{~Hz}$ line broadening. Only spectral regions downfield from the HDO resonance are shown and some key NOEs are identified. Several cross-peaks have been boxed-in and/or labeled to indicate intra- and inter- monomer $\alpha \mathrm{H}-\alpha \mathrm{H}$ NOEs which have been used to indicate $\mathrm{B}$ strand alignment. In Figure 3B, the cross-peaks are labeled with two numbers separated by a slash to indicate which residues in the sequence are involved. 


\section{NMR structure of CP-2}

Because initial NMR experiments indicated that CP-2 is well-folded, a complete NMR structure analysis of this peptide was performed. Figure $3 \mathrm{~A}$ shows the $\alpha \mathrm{H}-\mathrm{NH}$ region from a TOCSY plot of CP-2. Downfield resonance dispersion of $\mathrm{NH}$ and $\alpha \mathrm{H}$ resonances indicates the presence of well-folded $\beta$-sheet conformation, which is firmly evident by the observation of crossstrand $\alpha \mathrm{H}-\alpha \mathrm{H}$ NOEs (Figure 3B). Structurally, these NOESY data indicate the presence of the same anti-parallel $B$-sheet strand alignment as observed in Bpep-4 and other Bpep peptides. ${ }^{78}$ Figure 4A summarizes key cross-strand NOEs and gives the overall fold for CP-2. Since CP-2 through CP-6 peptides only differ in the position of the disulfide bridge, it is most probable that these CP peptides also share the same overall ß-sheet fold.
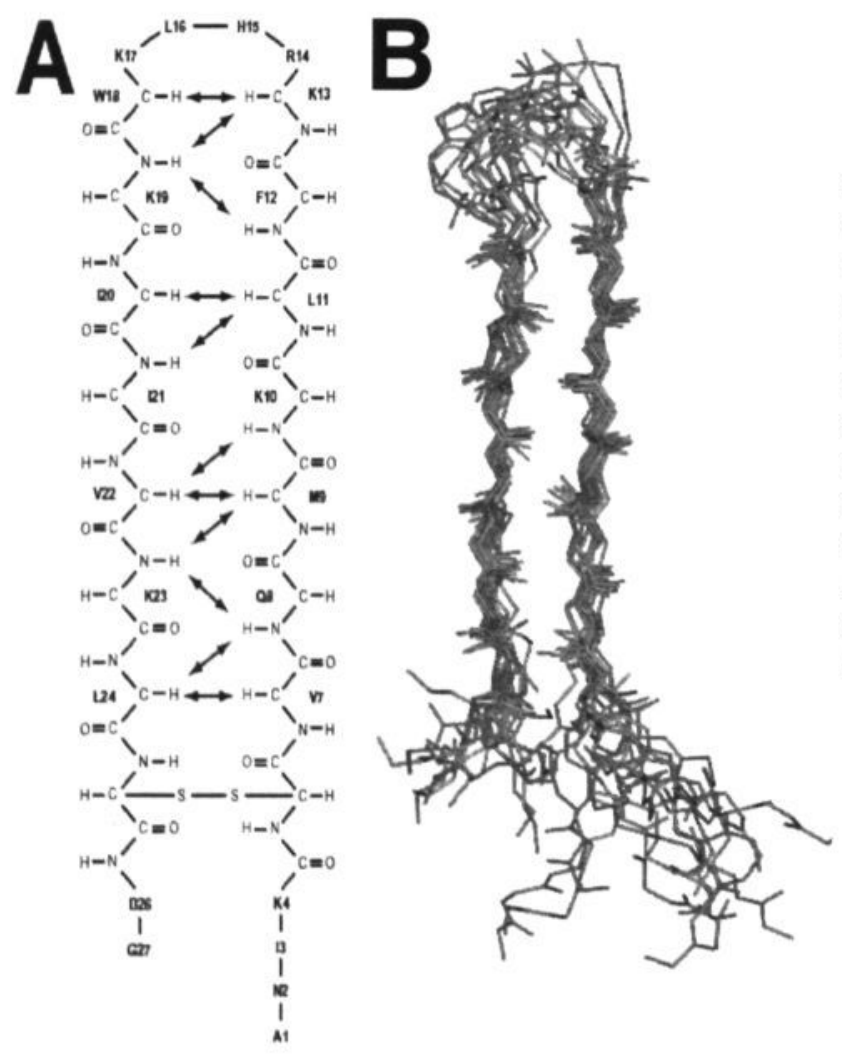

Figure 4. NOE-derived structures of CP2. Figure $4 \mathrm{~A}$ summarizes key cross-strand NOEs and gives the overall fold for CP-2. 19 final NOE-derived structures have been superimposed in Figure 4B and structural statistics are given in Table 1. The RMSD for backbone atoms of B-sheet residues 6-12 and 19-25 (excluding terminal and loop residues) is $0.61 \AA$ and for backbone atoms of residues 6-25 (excluding $\mathrm{N}$ - and $\mathrm{C}$ - terminal residues) it is 1.13 $\AA$. The best fit superpositions of backbone $\mathrm{C}_{\alpha}$ atoms for the final 19 structures are shown in Figure 4B.

For CP-2, conformational modeling was performed using NOE data acquired for the peptide. A total of 160 NOE distance constraints were derived from analysis of NOESY spectra. These include 94 intra-residue, 22 sequential, 16 medium-range $(|i-j|<5)$, and 28 long-range $(|i-j|>5)$ constraints. In addition, a total of 8 hydrogen bonds could be identified by inspection of initial CP-2 structures and from long-lived backbone $\mathrm{NHs}$, giving rise to 16 hydrogen bond distance constraints. The total number of experimentally derived constraints was therefore 168 , giving an average of 6 constraints per residue. The key cross-strand NOEs are shown in Figure 
4A. Initially, 100 structures for CP-2 were calculated as described in the Methods Section. The best fit superpositions of backbone $\mathrm{C} \alpha$ atoms for the final 19 structures are shown in Figure 4B. These structures showed no NOE violations greater $0.5 \AA$. Structural statistics (Table 1) show that the $\mathrm{N}$-terminus is somewhat less structurally-defined and that the structures satisfy experimental constraints quite well. In addition, $\phi$ and $\psi$ angular order parameters are all $>0.8$. Together, the above data indicate that these structures used to represent the solution conformation of CP-2 are well converged. The root mean square deviations (RMSDs) for backbone atoms of B-sheet residues 6-12 and 19-25 (excluding terminal and loop residues) is $0.61 \AA$ and for backbone atoms of residues $6-25$ (excluding $\mathrm{N}$ - and $\mathrm{C}$ - terminal residues) it is $1.13 \AA$.

Table 1. Structural statistics for the calculated structures of CP-2 from NMR data.

RMS Deviations from experimental distance restraints $(\AA)^{a}$

NOE (172)

$0.047 \pm 0.007$

H-bond (16)

Deviations from idealized geometry

Bonds $(\AA)$

Angles $\left({ }^{\circ}\right)$

0.003

Impropers $\left(^{\circ}\right)$

Energies (kcal.mol-1)

$0.5 \pm 0.02$

$0.37 \pm 0.02$

$E_{\text {NOE }}{ }^{b}$
$E_{\text {BOND }}$
$E_{\text {ANGLE }}$
$E_{\text {IMPROPER }}$
$E_{\text {TOTAL }}$

$5.1 \pm 0.62$

$3.8 \pm 0.63$

$39.4 \pm 2.8$

$19.2 \pm 5.7$

$80.2 \pm 10.3$

a None of the 19 final structures exhibited distance restraint violations greater than $0.5 \AA$ or dihedral angle violations greater than $5^{\circ}$. RMSD values represent the mean and standard deviations for the 19 structures.

'The final values of the NOE $\left(E_{\text {NOE }}\right)$ was calculated with a force constants of $50 \mathrm{kcal} \cdot \mathrm{mol}^{-1} \cdot \AA^{-2}$.

\section{CP peptides inhibit endothelial cell proliferation}

CP peptides were tested for their ability to inhibit the proliferation of growth factor-induced (bFGF, $10 \mathrm{ng} / \mathrm{mL}$ ) HUVECs in the $\left[{ }^{3} \mathrm{H}\right]$-thymidine incorporation assay. Relative to the activity of parent peptide angine $x^{36}, \mathrm{CP}$ peptides effectively inhibited the proliferation of EC to various extents. Percentage EC proliferation vs. peptide concentration is plotted in Figure 5. While the linear control peptide, CP-1, and three of the disulfide-bridged variants, CP-4, CP-5 and CP-6, were essentially as effective as anginex, CP-2 and CP-3 appeared to be almost as active, and control peptides CP-7 and CP-8 were only slightly active. The kinetics of these anti-proliferative effects on EC were similar to that found for anginex, with half-maximal effects after 36 to 40 hours and maximal responses after 3 days. As with anginex, the inhibition of proliferation by CP peptides was specific for EC since proliferation of fibroblasts obtained from human endometrium was unaffected (data not shown). 


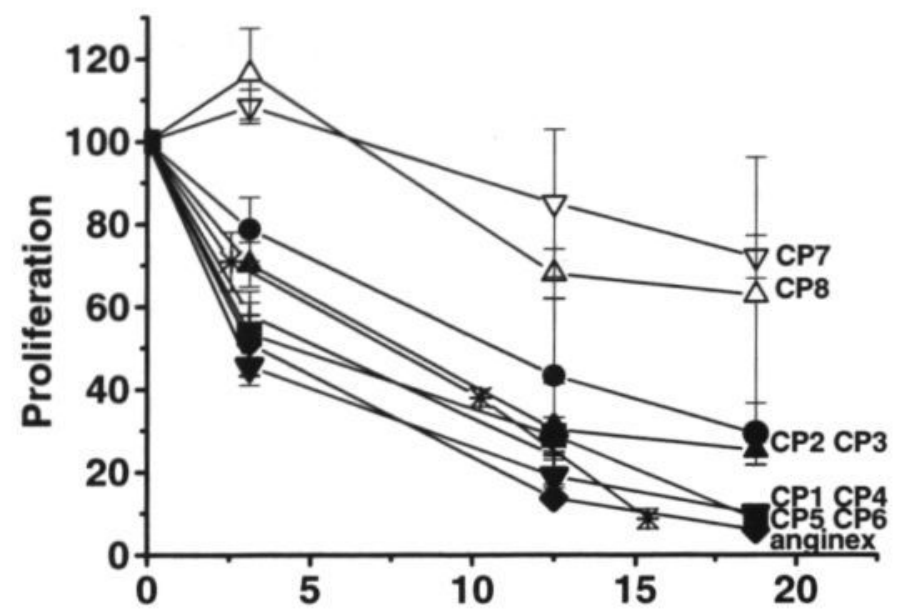

Figure 5. Inhibition of EC proliferation by CP peptides. Proliferation of bFGFstimulated $(10 \mathrm{ng} / \mathrm{ml})$ EC cultures is measured by quantification of ${ }^{3} \mathrm{H}$ thymidine incorporation. Proliferation is expressed as mean counts per minute (cpm) of quadruplicate cultures of four independent experiments. Dose-response curves of $\mathrm{CP}$ peptides and parent anginex on bFGF-stimulated HUVEC proliferation are shown. The error bars represent the standard error of the mean (SEM).

\section{Concentration $(\mu \mathrm{M})$}
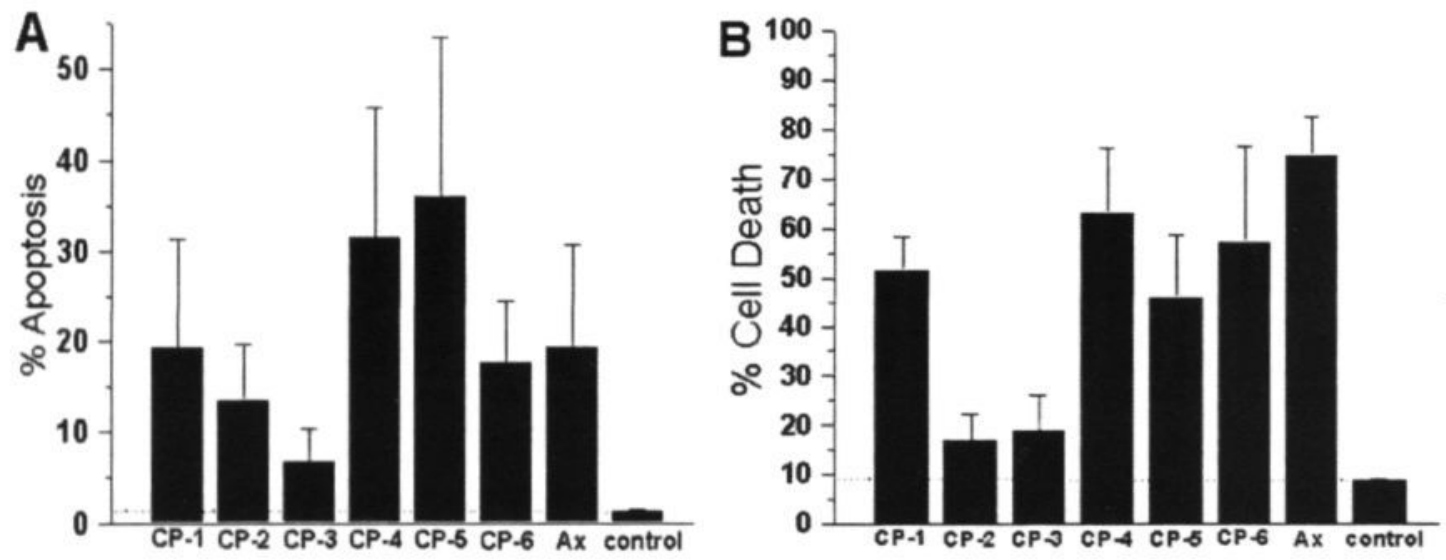

Figure 6. CP peptides induce apoptosis in EC. HUVECs were cultured for 2 days in the presence or absence of $25 \mu \mathrm{MCP}$ peptides or anginex. (A) Apoptosis induction was demonstrated by analysis of subdiploid cells after DNA extraction and staining with propidium iodide. (B) Total number of dead cells is determined by total number of diploid cells as quantified by FACS analysis. Quantification of the means of four different experiments is shown. The error bars represent the standard error of the means (SEM).

\section{CP peptides promote apoptosis in EC}

To investigate whether CP peptide-induced inhibition of EC growth is based on specific induction of apoptosis in these cells, as was demonstrated for angine ${ }^{36}$, EC were exposed to $25 \mu \mathrm{M}$ peptide for 48 hours, and the percentage of cells undergoing apoptosis was quantified by analysis of DNA fragmentation following DNA extraction, propidium iodide staining and flow cytometric analysis. Although all CP peptides demonstrated apoptotic activity, CP-4 and CP-5 were the most active and even slightly more effective, on average, than anginex (Figure 6A). Since apoptosis in this assay is defined as those subdiploid cells with a minimum of $10 \%$ of the DNA content of the diploid cells, advanced or late stage apoptosis may result in less than $10 \%$ of 
that value. Therefore, the total number of dead cells is shown in Figure 6B. The loss of ECs in $\mathrm{G}_{2}$ and $\mathrm{M}$ ( 4 DNA) phases of the cell cycle is about $10 \%$ or less (data not shown). The correlation between apoptosis induction and anti-proliferative effects suggests that CP peptides, like anginex, regulate EC growth by forcing EC to undergo apoptosis.

\section{In vitro angiogenesis assay}

Since angiogenesis is a complex process, which, aside from EC proliferation, depends on cell migration and differentiation, the effects of $\mathrm{CP}$ peptides on angiogenesis were investigated in an in vitro collagen matrix-based sprout formation assay. ${ }^{154}$ Although all CP peptides were able to inhibit sprout formation to various extents (Figure 7), the best responses, comparable to that from anginex, were observed with $\mathrm{CP}-1$ and $\mathrm{CP}-2$.

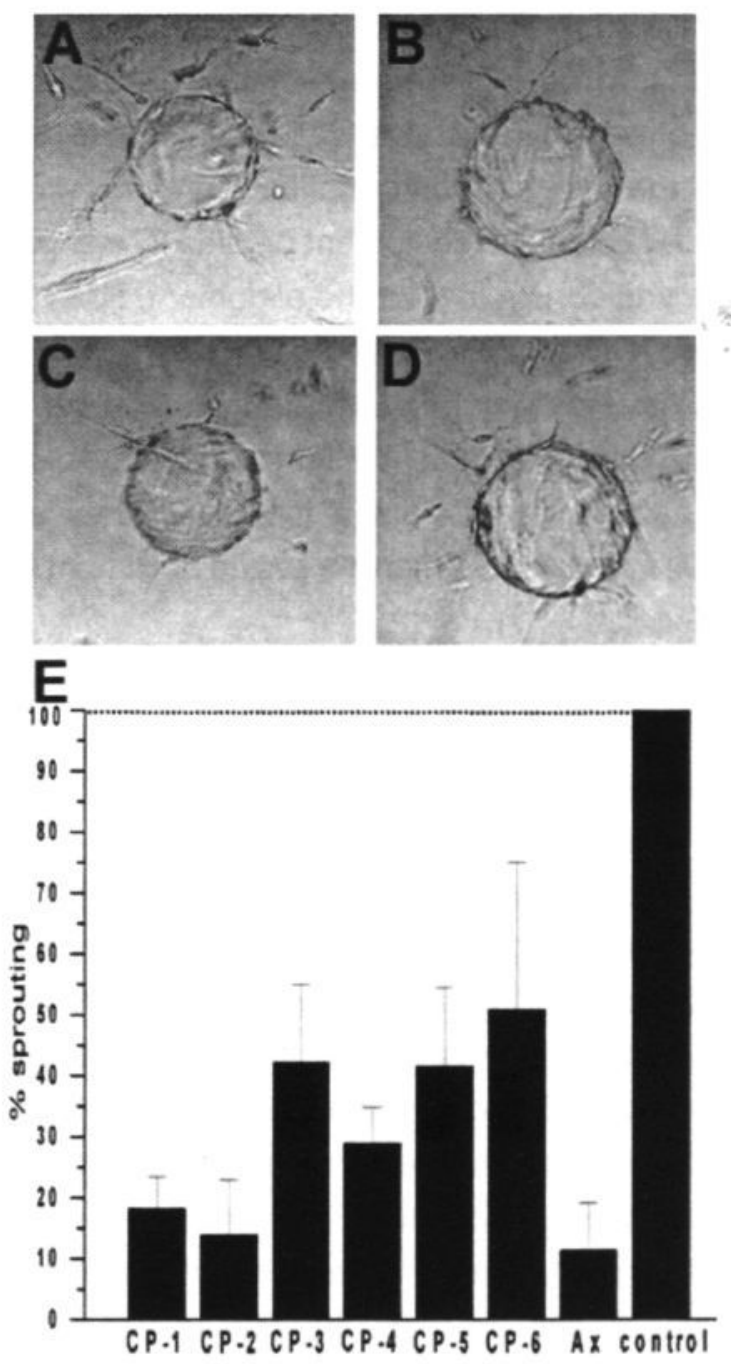

Figure 7. Anginex inhibits in vitro angiogenesis in a collagen matrix. BCE were cultured on gelatin coated Cytodex- 3 beads in a collagen matrix. Sprouting was induced by addition of $20 \mathrm{ng} / \mathrm{ml} \mathrm{bFGF}$ and $25 \mu \mathrm{M}$ of the CP peptides or anginex was added when tested. Upper panels represent tube formation under control conditions ( $A$, pixel count 1002), in the presence of anginex (B, pixel count 211), CP-1 (C, pixel count 205) or CP-6 (D, pixel count 513). CP7 had a pixel count of 990. The beads have a mean size of $170( \pm 40)$ microns. Quantification, performed by using NIH-image software, of the means of four different experiments is shown in panel E. The error bars represent the standerd error of the mean (SEM). 


\section{Discussion}

The design of novel peptides is a powerful tool in the development of biologically active molecules. Anginex (Bpep-25), which is one such designed peptide ${ }^{77}$, functions essentially as a cytokine to specifically inhibit EC proliferation and angiogenesis ${ }^{36}$ and thereby attenuate tumor growth ${ }^{154-156}$. The main goal of structural biologists and pharmaceutical chemists working to develop better and more effective anti-angiogenic anti-tumor agents is the identification of specific amino acid residues and their spatial relationships that are crucial to promoting anti-angiogenic effects at the level of molecular interactions. Nevertheless, for anti-angiogenic proteins, such structureactivity relationships are sorely needed, and even the analysis of high-resolution molecular structures of a number of anti-angiogenic proteins, e.g., endostatin ${ }^{47}, \mathrm{PF}^{172}$ and $\mathrm{BPI}^{52}$, has not provided this information.

In the present paper, our primary goal was to define the biologically active conformation of anginex. Although previous biophysical studies on anginex indicate that the peptide forms a $\beta$ sheet conformation, that conformation is not well-folded and leaves open the question of the bioactive structure. On the assumption that the bioactive conformation of anginex would be as depicted in Figure 1, disulfide bonds were designed into equipotent peptide 27 mer variants of anginex that limit its conformational space and constrain $\beta$-strand alignment. NMR structure analysis showed that one of these peptides, CP-2, is well-folded as an anti-parallel $\beta$-sheet (Figure 4). Since the other CP peptides only differ in the position of the disulfide bridge, it is most probable that all CP peptides share the same overall $B$-sheet fold. Functionally, these disulfideconstrained peptides essentially exhibit the same anti-angiogenic activity as anginex, as long as the proposed $\beta$-strand alignment (Figure 1) is maintained. When the strand alignment is shifted, as in CP-7 and CP-8, bioactivity falls significantly.

Mechanistically, the correlation between apoptosis induction and anti-proliferative effects suggests that $\mathrm{CP}$ peptides, like anginex, regulate $\mathrm{EC}$ growth by forcing $\mathrm{EC}$ to undergo apoptosis. However, in the in vitro angiogenesis sprouting assay, inhibitory activity at the level of anginex was observed only for CP-1 and CP-2. This was surprising because CP-4, CP- 5 and CP- 6 were the most effective at inhibiting EC proliferation. One reason for this could be the complexity of the collagen gel sprouting assay itself. Because these peptides must first navigate through the gel before exerting their effect on ECs, it may be that CP-1 and CP-2 permeate the gel better than other CP peptides or interact somewhat differently with the gel matrix. Alternatively, it may be that the sprouting assay is indeed the more sensitive indicator of anti-angiogenic potential. In any event, the general conclusion remains that anti-parallel $ß$-sheet with a specific $B$-strand alignment is the conformation of the peptide required for full bioactivity. This finding is consistent with earlier results on anginex that in the ß-sheet conformation all five hydrophobic residues (L5, V7, I20, V22, L24), which were identified as being crucial to anti-proliferative activity, are proximal and are conformed on the hydrophobic face of the amphipathic $\beta$-sheet. ${ }^{95}$ Comparison of the 
three-dimensional structures of several anti-angiogenic proteins, e.g. endostatin ${ }^{47}$, PF4 ${ }^{172}$, tumor necrosis factor- $\alpha^{173}$ and $B P I^{52}$, provides a higher level of structural commonality among antiangiogenic proteins in that they are comprised primarily of anti-parallel $B$-sheet structure. Furthermore, a survey of amino acid sequences from numerous anti-angiogenic proteins reveals that they are compositionally similar, containing numerous hydrophobic and cationic residues. These structural and compositional characteristics, which appear to be functionally important, are embodied in anginex ${ }^{95}$ As structure-function relationships in these and other anti-angiogenic proteins and peptides become known, it will be interesting to see if this is a common feature among anti-angiogenic proteins. Knowing that this $\beta$-sheet conformation is that which promotes anti-angiogenic activity, will aid in the development of small-molecule mimetics of the anginex peptide. 


\section{Chapter 6}

\section{Design of a partial-peptide mimetic of anginex with anti-angiogenic and anti-cancer activity}

Kevin H. Mayo, Ruud P.M. Dings, Carolee Flader, Irina Nesmelova, Balasz Hargittai, Daisy W.J. van der Schaft, Loes I. van Eijk, Dinesha Walek, Judy R. Haseman, Thomas R. Hoye, and Arjan W. Griffioen 


\section{Abstract}

Based on structure-activity relationships of the angiostatic $B$-sheet-forming peptide anginex, we have designed a mimetic, $6 \mathrm{DBF} 7$, which inhibits angiogenesis and tumor growth in mice. 6DBF7 is composed of a B-sheet-inducing dibenzofuran (DBF) turn-mimetic and two short key amino acid sequences from anginex. This novel anti-angiogenic molecule is more effective in vivo than parent anginex. In a mouse xenograft model for ovarian carcinoma, $6 \mathrm{DBF} 7$ is observed to reduce tumor growth by up to $80 \%$. It is suggested that the activity is based on anti-angiogenesis because in vitro tube formation is inhibited and because treatment of tumor-bearing mice led to a significant reduction in microvessel density within the tumor. This partial peptide-mimetic is the first EC-specific molecule designed as a substitute for an angiostatic inhibitory peptide. 


\section{Introduction}

Angiogenesis, the process of new blood vessel formation, is key to normal organ development, as well as to various pathological disorders like cancer, arthritis, diabetic retinopathy and restenosis. ${ }^{96}$ The use of agents that can inhibit angiogenesis, particularly in anti-tumor research (e.g. refs 9 and 174), has indicated that anti-angiogenic therapy can be a promising therapeutic modality in the future. To date, the search for angiogenesis inhibitors has been focused on controlling two of the processes that promote angiogenesis: endothelial cell (EC) growth and EC adhesion. ${ }^{10,121}$ Targeting EC as anti-tumor treatment is attractive primarily because EC are more accessible than are other cells to pharmacologic agents delivered via the blood, and EC are genetically stable and are not easily mutated into drug resistant variants. Most antiangiogenic agents have been discovered by identifying endogenous molecules, primarily proteins, that inhibit EC growth. This traditional approach has produced a number of anti-angiogenics, such as platelet factor-4 (PF4) ${ }^{103}$, thrombospondin ${ }^{175}$, interferon- $\gamma$ inducible protein- $10^{125}$, angiostatin ${ }^{8}$, endostatin and restin ${ }^{9.43}$ and bactericidal-permeability increasing (BPI) protein ${ }^{82}$.

Numerous angiostatic compounds have been identified, many of which are currently in various phases of clinical cancer trials (http://cancertrials.nci.nih.gov). Although a number of compounds have shown promise in the clinic, no major breakthroughs have been reported using antiangiogenic agents as stand-alone therapy. This underscores the need for more and better angiostatic compounds for use as stand-alone or in combination with conventional therapies. Moreover, some angiogenesis inhibitors have been shown to be ineffective or to cause diverse and multiple unwanted biological side-effects. This latter phenomemon was, to some extent, expected as many of these endogenous angiostatic proteins are pleiotropic, i.e., they are involved in several biological processes. Often these functions are found to arise from separate sites on the molecule and thus can be separated using smaller segments or domains of a given protein. This relates to the search for new anti-angiogenic agents, which is aimed at improving specificity, reducing the therapeutic dosage, and minimizing molecular size with a small-molecule anti-angiogenic agent or anti-angiogenic protein mimetic. This is one of the main goals among structural biologists and pharmaceutical chemists working to produce novel anti-angiogenic agents. For the design of small molecule mimetics of anti-angiogenic proteins, the identification of specific, functionally key amino acid residues and their spatial relationships is crucial. Nevertheless, structure-activity relationships (SARs) for anti-angiogenic proteins are sorely needed, and even the analysis of high-resolution molecular structures of a number of anti-angiogenic proteins, e.g., endostatin ${ }^{47}$, PF $4^{172}$ and $\mathrm{BPI}{ }^{52}$, has yet to provide this information.

Recently, we reported the design of the anginex peptide, a cytokine-like $B$-sheet-forming peptide $33 \mathrm{mer}^{95}$, which is a potent inhibitor of angiogenesis ${ }^{36}$ and tumor growth ${ }^{154-156}$. Anginex is more effective at inhibiting EC growth than PF4 and several other well-known angiogenesis inhibitors such as angiostatin, endostatin, AGM-1470 and thrombospondin-1. ${ }^{36}$ This peptide 
33mer acts by specifically blocking adhesion and migration of angiogenically-activated EC, leading to apoptosis and ultimately to inhibition of angiogenesis in vitro and in vivo and inhibition of human and murine tumor growth by up to about $80 \%$ in various models. ${ }^{154-156}$ Anginex is an amphipathic $B$-sheet-forming peptide ${ }^{95,176}$, and because the relatively small size of anginex, a complete SAR is easier to perform than with larger known anti-angiogenic proteins like those mentioned above. Here, we report for the first time the design of a partial-peptide mimetic of anginex that was based on the identification of functionally key residues and the conformation responsible for the anti-angiogenic activity of anginex.

\section{Material and Methods}

\section{Peptide synthesis}

Peptides were synthesized using a Milligen/Biosearch 9600 peptide solid-phase synthesizer using fluorenylmethoxycarbonyl (Fmoc) chemistry. Lyophilized crude peptides were purified by preparative reversed-phase HPLC on a C18 column with an elution gradient of $0-60 \%$ acetonitrile with $0.1 \%$ trifluoroacetic acid in water. The purity and composition of the peptides were verified by HPLC (Beckman Model 6300) analysis of amino acid composition of hydrolysates prepared by treating the peptides under argon in $6 \mathrm{~N} \mathrm{HCl}$ for 24 hours at $110^{\circ} \mathrm{C}$. The amino acid sequences of peptides were confirmed by $\mathrm{N}$-terminal sequencing and mass spectrometry.

\section{Synthesis of DBF analogs}

Unexceptional phases of solid-phase peptide synthesis (SPPS) were carried out on an ABI (Applied Biosystems, Inc.) 431 peptide synthesizer using Fmoc methodology and BOP/ $\mathrm{HOBT}$ as coupling reagents. Fmoc-DBF- $\mathrm{CO}_{2} \mathrm{H}$ (1) was prepared by slight modification of the reported method. ${ }^{17}$ In this nine-step synthesis, the intermediates and final product were characterized by TLC and ' $\mathrm{H}$ NMR, with GC-LRMS, ${ }^{13} \mathrm{C}$ NMR, $\mathrm{mp}$, and IR being used as appropriate. Coupling of 1 to $\mathrm{I} 20$ as well as of $\mathrm{L} 11$ to the peptide-DBF- $\mathrm{NH}_{2}$ was performed on the synthesizer. Coupling of Fmoc-K10- $\mathrm{CO}_{2} \mathrm{H}$ to the peptide-DBF-L11- $\mathrm{NH}_{2}$ sequence was difficult and required manual SPPS using the more reactive HATU reagent. ${ }^{178}$ The remaining couplings required for production of 2DBF7 through 11DBF7 were carried out using BOP/HOBT conditions on the peptide synthesizer. After the final Fmoc deprotection, each of the DBF peptides was released from the resin with simultaneous removal of all acidolyzable trityl and tert-butyl side-chain protecting groups using Reagent K. ${ }^{179} \mathrm{~A}$ Rink amide or similar resin was used to provide the primary amide form of the C-terminal D24 unit. Lyophilized crude peptides were purified by HPLC as described above. Purity and composition of the peptides were verified by analytical HPLC, matrix assisted laser desorption ionization (MALDI) mass spectrometry using a Hewlett-Packard 
G2025A system and sinapinic acid as matrix, and analysis of amino acid composition of hydrolysates $\left(6 \mathrm{~N} \mathrm{HCl}, 110^{\circ} \mathrm{C}, 24 \mathrm{~h}\right.$, under argon).

\section{NMR spectroscopy}

For NMR measurements, freeze-dried DBF analogs were dissolved in water-DMSODPC mixture. Peptide concentration was $3 \mathrm{mM}$. $\mathrm{pH}$ was adjusted to $\mathrm{pH} 5.7$ by adding $\mu \mathrm{L}$ quantities of $\mathrm{NaOD}$ or $\mathrm{HCl}$ to the peptide sample. NMR spectra were acquired on a Varian UNITY Plus-600 NMR spectrometer at $25^{\circ} \mathrm{C}$.

2D-homonuclear TOCSY with DIPSI ${ }^{180}$ spinlock (mixing time $80 \mathrm{~ms}$ ) was used to identify spin systems. 2D NOESY experiments ${ }^{160}$ were performed for sequential assignments and conformational analysis. WATERGATE ${ }^{181,182}$ was used to attenuate the water resonance. Spectra were collected as 256 to $512 \mathrm{t} 1$ experiments, each with 2048 complex data points over a spectral width of $6 \mathrm{kHz}$ in both dimensions with the carrier placed on the water resonance. 16 scans were time averaged per $\mathrm{t} 1$ experiment. Data were processed directly on the spectrometer using VNMR (Varian, Inc., Palo Alto) or NMRPipe ${ }^{163}$ on an SGI workstation.

\section{Structural modeling}

Analysis of NOE growth curves indicated that backbone to backbone inter-proton NOEs were normally maximum at about $200 \mathrm{~ms}$. Interproton distance constraints were derived from NOEs assigned in ${ }^{1} \mathrm{H}$ NOESY spectra acquired with mixing times of $100 \mathrm{~ms}$. NOEs were classified as strong, medium, weak or very weak corresponding to upper bound distance constraints of 2.3, 2.8, 3.5, and $4.5 \AA$, respectively. The lower bound restraint between non-bonded protons was set to $1.8 \AA$. Pseudo-atom corrections were added to the upper bound distance constraints where appropriate, and a $0.5 \AA$ correction was added to the upper bound for NOEs involving methyl protons. Hydrogen bond constraints were identified from the pattern of sequential and interstrand NOEs involving $\mathrm{NH}$ and $\mathrm{C} \alpha \mathrm{H}$ protons, together with evidence of slow amide protonsolvent exchange. Each hydrogen bond identified was defined using two distance constraints; $\mathrm{r}_{\mathrm{NH}-\mathrm{O}}=1.8$ to $2.5 \AA$, and $\mathrm{r}_{\mathrm{N}-0}=1.8$ to $2.5 \AA$.

Derived internuclear distance constraints were used in calculating structures for 6DBF7 by using X-PLOR. ${ }^{164}$ The molecule was created and a template coordinate set was generated by using the Template routine. The ab initio simulated annealing (SA) protocol was then used. The SA procedure ran high temperature dynamics ( $3000 \mathrm{~K}$ for $120 \mathrm{ps})$ and then cooled down to 100 $\mathrm{K}$ in $50 \mathrm{~K}$ steps with $1.5 \mathrm{ps}$ molecular dynamics at each step. Powell minimization was performed at $100 \mathrm{~K}$ for 1000 steps. Structure refinement was done based on simulated annealing starting at $1000 \mathrm{~K}$ and ending at $100 \mathrm{~K}$. Final structures were subjected to the X-PLOR Accept routine with the violation threshold for NOEs of $0.5 \AA$ and dihedral angles of $5^{\circ}$. Angles, bond lengths or impropers were not allowed to deviate from ideal geometry more than $5^{\circ}, 0.05 \AA$ and 
$5^{\circ}$, respectively. Structures were superimposed using the BIOSYM INSIGHT viewer (Molecular Simulations, Inc.) and were analyzed using X-PLOR analysis routines.

\section{Cells, cultures and reagents}

Human umbilical vein derived EC (HUVEC) were harvested from normal human umbilical cords by perfusion with $0.125 \%$ trypsin/EDTA. Harvested HUVECs were cultured in gelatin coated tissue culture flasks and subcultured 1: 3 once a week in culture medium (RPMI-1640 with 20\% human serum (HS), supplemented with $2 \mathrm{mM}$ glutamine and $100 \mathrm{U} / \mathrm{ml}$ penicillin and $0,1 \mathrm{mg} / \mathrm{ml}$ streptomycin). Bovine capillary EC (BCE) were kindly provided by Dr. M. Furie (State University of New York, Stony Brook, USA) and were cultured in fibronectin coated tissue culture flasks in RPMI1640 medium containing $10 \%$ FCS, glutamine and antibiotics.

\section{Proliferation measurement}

EC proliferation was measured using a $\left[{ }^{3} \mathrm{H}\right]$-thymidine incorporation assay. Proliferation of bFGF-stimulated ( $10 \mathrm{ng} / \mathrm{ml}$ ) HUVEC cultures was measured by quantification of ${ }^{3} \mathrm{H}$-thymidine incorporation. Proliferation is expressed as mean counts per minute $(\mathrm{cpm})$ of quadruplicate cultures in three independent experiments ( \pm SEM). EC were seeded at 5000 cells/well in flatbottomed tissue culture plates and grown for 3 days in the absence or presence of regulators, in culture medium. During the last 6 hours of the assay, the culture was pulsed with $0.5 \mu \mathrm{Ci}$ [methyl${ }^{3} \mathrm{H}$-thymidine/well.

\section{In vitro angiogenesis assay}

Sprouting and tube formation of BCE were studied using cytodex- 3 beads overgrown with BCE in a 3-dimensional collagen gel (vitrogen-100, Collagen Corp., Fremont, CA, USA) as described by van der Schaft et al.. ${ }^{132}$ Following gelation, culture medium containing $20 \mathrm{ng} / \mathrm{mL}$ bFGF, with or without anginex or DBF analogs, was applied on top of the gel. Photograps were made after 24 hours of cell culture at $37^{\circ} \mathrm{C}$. The amount of sprouting in each well (i.e. the total length of the sprouts) was quantified by the computer program NIH image. To quantify differences in sprouting and tube formation, statistical analysis was performed using the Mann-Whitney U test.

\section{Tumor model studies in athymic (nude) mice}

In all studies, female athymic nude mice (nu/nu, 5-6 weeks old) were used. These mice were purchased from the National Cancer Institute and allowed to acclimatize to local conditions for at least one week. Animals were given water and standard chow ad libitum, and were kept on a 12 hour light/dark cycle. All experiments were approved by the University of Minnesota Research Animal Resources ethical committee. Mice were randomized and split into three groups: 1) human serum albumin (10 mg/kg/day), 2) anginex (10 mg/kg/day) and 3) DBF analog (10 mg/ 
$\mathrm{kg} /$ day). Compounds were diluted in $100 \mathrm{mM} \mathrm{SDS}$ and administered using osmotic mini-pumps (Durect, Cupertino, CA). Exponentially growing MA148 human ovarian carcinoma cells, kindly provided by Prof. Dr. Ramakrishnan ${ }^{155}$, were cultured in RPMI 1640 medium (Life Technologies, Grand Island, NY). This medium was supplemented with $10 \%$ fetal bovine serum and $1 \%$ penicillin/streptomycin (Cellgro, Mediatech, Washington, DC) at $37^{\circ} \mathrm{C}$ and $5 \% \mathrm{CO}_{2} .100 \mu \mathrm{l}$ of this tumor cell suspension $\left(2 \times 10^{7} \mathrm{cells} / \mathrm{ml}\right)$ was then injected subcutaneously into the right flank of each mouse. Pumps were implanted into the left flank of mice for subcutaneous administration of compound over a 28-day treatment span.

Two variants of this model were used: prevention and intervention. For the prevention variant, treatment was initiated at the time of inoculation with MA148 cells. For the intervention variant, tumors were allowed to grow to an average size of $50 \mathrm{~mm}^{3}$ (usually day 7 post-inoculation) before treatment was initiated. With either variant, animals were randomized prior to the initiation of treatment. Treatment was administered via osmotic mini-pumps, which were implanted subcutaneously in the left flank of mice. Concentrated solutions of anginex or DBF analogs were formulated such that the 28-day treatment period would be covered by implantation of a single pump. In each study, control groups of animals were administered either PBS or PBS containing human serum albumin. Tumor growth curves were found to be virtually identical in either of these control cases.

Tumor volume was determined by measuring the diameters of tumors using calipers (Scienceware, Pequannock, NJ) using the equation for the volume of a spheroid: $\left(a^{2} \times b \times \Pi\right) / 6$, where ' $a$ ' is the width and ' $b$ ' the length of the tumor. Measurements were performed two or three times per week. At the conclusion of an experiment, tumor weights were also taken following excision of the tumors from euthanized animals. Tumor weights correlated well with tumor volumes calculated in this way.

\section{Immunohistochemistry}

Immunohistochemistry was used to assess microvessel density and the extent of total cell apoptosis. Tumor tissue was embedded in tissue freezing medium (Miles Inc, Elkart, IN) and shock frozen in liquid nitrogen. Sections of tissue ( $10 \mu \mathrm{m}$ thickness) were prepared for immunohistochemical analysis. For this, tissue sections were brought to room temperature, air dried overnight, and then fixed in acetone for 10 minutes. Slides were allowed to air dry for at least 30 minutes and were washed three times for 5 minutes each in phosphate-buffered saline (PBS, pH 7.4). Samples were then blocked with PBS containing $0.1 \%$ bovine serum albumin and $3 \%$ human serum albumin for at least 30 minutes at room temperature in a humidified box. Samples were subsequently incubated with phyco-erytrin (PE)-conjugated monoclonal antibody to CD31 (PECAM-1) in a 1:50 dilution (Pharmigen, San Diego, CA) to stain for microvessel density. After 
1-hour incubation at room temperature, slides were washed with PBS and immediately imaged using an Olympus BX-60 fluorescence microscope at 200X magnification.

To assess the extent of total cell apoptosis, tissue sections were stained by using the TUNEL (terminal deoxyribonucleotidyl transferase-mediated dUTP-nick-end labeling) assay, which was performed according to the manufacturer's instructions (in situ cell death detection kit, fluorescein; TUNEL, Roche). Digital images were stored and processed using Adobe Photoshop (Adobe Inc., Mountain View CA). Quantification of microvessel density, the rate of proliferation and total cell apoptosis were determined as described earlier. ${ }^{12}$ Statistical analysis was performed using the Student's t-test.

\section{Toxicity assays}

As an indirect measurement of general toxicity, body weights of mice were monitored twice weekly, using a digital balance (Ohaus Florham, NJ). To determine hematocrit and creatinine levels, blood samples were extracted by tail vein bleedings one day after terminating treatment and blood was collected in heparinized micro-hematocrit capillary tubes (Fisher; Pittsburgh, PA). For hematocrit levels, samples were spun down for 10 minutes in a micro-hematocrit centrifuge (Clay-Adams; NY), and the amount of hematocrit was determined using an international microcapillary reader (IEC; Needham, Mass). To obtain creatinine levels, a kit was purchased from Sigma (Sigma Diagnostics; St Louis, MO) and used according to the manufacturer's instructions.

\section{Results}

\section{Design of a partial-peptide mimetic}

For input into designing the mimetic, we first performed structure-activity studies on anginex. Working with this relatively small peptide 33mer allowed for a thorough assessment of functionally-key residues by using alanine scanning and walk-through variants. The relative anti-proliferative effect of alanine-substituted analogs of anginex against EC is plotted in Figure 1A. Residues that demonstrate the most significant drop in the ability of anginex to inhibit EC proliferation are hydrophobic residues within the first two $B$-strands: I3, L5, V7, L11 and I20. Conformationally, these functionally key hydrophobic residues all lie on the same face of the amphipathic antiparallel B-sheet. ${ }^{95.176}$ Also shown in Figure 1A are anti-proliferation activities of eight walk-through dodecapeptides that successively sample segments of the anginex sequence, shifting three residues in each peptide. Only three walk-through peptides ( $\mathrm{IC}_{50}$ values listed at the right) demonstrate any significant anti-proliferative activity relative to parent anginex. As with results from alanine scanning, these peptides also encompass $B$-strands 1 and 2 . Based on these results, 
we concluded that anti-angiogenic activity is localized within $\beta$-strands 1 and 2 and that residues in turns 1 and 2 and $B$-strand 3 are functionally dispensable. For orientation, the $B$-strand alignment for anginex is depicted in Figure 1B; portions of the molecule containing the key sequences are boxed.

Using this information, we designed the DBF-series of partial-peptide mimetics, in which B-strand 3 and turn 2 of anginex were omitted and a dibenzofuran (DBF) B-turn mimetic ${ }^{183,184}$ was introduced, replacing turn 1 and residues $F 12, K 13$ and $W 18, K 19$ of $B$-strands 1 and 2, respectively. The DBF $\beta$-turn mimetic was used in order to maintain the bioactive $\beta$-sheet conformation of anginex. ${ }^{176}$ The parent DBF-based compound depicted in Figure $1 \mathrm{C}$ is called 11DBF7, where numbers at the left and right of DBF refer to the number of amino acid residues in the $\mathrm{N}$ - and $\mathrm{C}$ terminal strands, respectively, from anginex. To identify the shortest sequences required for bioactivity, a series of $\mathrm{N}$ - and $\mathrm{C}$-terminal deletion variants of 11DBF7 was made as listed in Table 2.

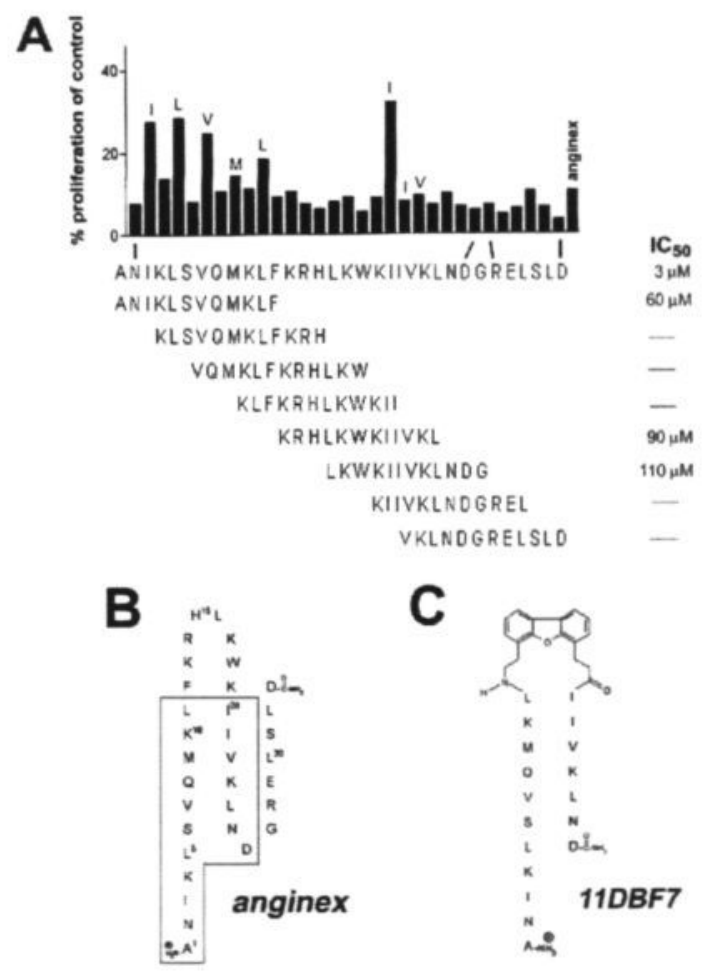

Figure 1. Effects of alanine scan and walkthrough variants of anginex on EC proliferation in vitro. (A) The sequence is shown for anginex in single letter codes of amino acid residues, below which are given the amino acid sequences for the dodecapeptide walk-throughs of anginex. Proliferation of bFGF-stimulated (10 ng/ $\mathrm{ml}$ ) human umbilical vascular EC (HUVEC) cultures was measured by quantification of ${ }^{3} \mathrm{H}$-thymidine incorporation. Proliferation is expressed as mean counts per minute (cpm) of quadruplicate cultures in three independent experiments ( \pm SEM). EC proliferation results from alanine scanning (tested at $25 \mu \mathrm{M}$ dose) are expressed in bar graph format as the percentage of proliferating EC \{ the arithmetic mean counts per minute (cpm) of triplicate cultures\} relative to control cultures. For walk-through peptides, results are given as $\mathrm{IC}_{50}$ values from dose response curves; only $\mathrm{IC}_{50}$ values for dodecapeptides with significant activity relative to anginex are given. (B) The overall fold for anginex ( $3 \mathrm{~B}$-strands with 2 turns) is shown with functionally key residues boxed. (C) The parent DBF analog is depicted with the introduction of the scaffold and the two functionally key Bstrand sequences from anginex. 
Table 1. Structural statistics for NOE-derived structures of 6DBF7.

RMS Deviations from experimental distance restraints $(\AA)^{a}$

NOE (168)

$0.1 \pm 0.01$

$\mathrm{H}$-bond (16)

Deviations from idealized geometry

Bonds $(\AA)$

Angles $\left({ }^{\circ}\right)$

Impropers $\left(^{\circ}\right)$

Energies (kcal.mol-1)

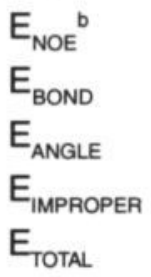

$0.0071 \pm 0.0004$

$1.0 \pm 0.03$

$0.6 \pm 0.05$
$13.2 \pm 1.4$

$71.8 \pm 4.2$

$7.4 \pm 1.3$

$202.6 \pm 22.4$

"None of the 28 final structures exhibited distance restraint violations greater than $0.5 \AA$ or dihedral angle violations greater than $5^{\circ}$. RMSD values represent the mean and standard deviations for the 28 structures.

${ }^{b}$ The final value of the NOE $\left(E_{\mathrm{NOE}}\right)$ was calculated with a force constant of $50 \mathrm{kcal} \cdot \mathrm{mol}^{-1} \cdot \AA^{-2}$.

\section{B-sheet structure is preserved in DBF analogs}

We used NMR spectroscopy to investigate whether B-sheet conformation was preserved in DBF analogs. Analogs 11DBF7 and 6DBF7 (that has more equivalent B-strand lengths) were the focus of this structural study. Due to their limited water solubility and the desire to mimic a membrane-like environment, these compounds were investigated in dodecylphosphocholine (DPC) micelles. At the millimolar concentrations required for NMR work, 11DBF7 gave overlapping resonances that made spectral analysis ambiguous, whereas 6DBF7 gave excellent NMR spectra that allowed complete structure analysis. NOEs and coupling constants diagnostic of anti-parallel B-sheet conformation were readily identified and used in computational modeling. The superimposed 28 lowest energy structures (Figure 2A) have a backbone RMSD value (Nand C-terminal residues excluded) of $0.11 \AA$. Additional structural statistics are given in Table 1. A simplified illustration of this folding pattern that highlights residues on both hydrophobic and hydrophilic surfaces of the B-sheet (Figure 2B). In this orientation of the molecule, the dibenzofuran moiety is lying on edge. Notice also that two aliphatic hydrophobic residues leucine (L11) and isoleucine (I20) from anginex are packed against the phenyls of the DBF group in 6DBF7 (Figure $2 \mathrm{~B}$ ). In effect, this sets up and helps to stabilize the $B$-sheet fold. ${ }^{17}$ Based on this structural information, we concluded that other DBF analogs would fold similarly, albeit to various extents depending on the lengths of the two strands, i.e., strands of equal length are expected to be better able to form $B$-sheets. 


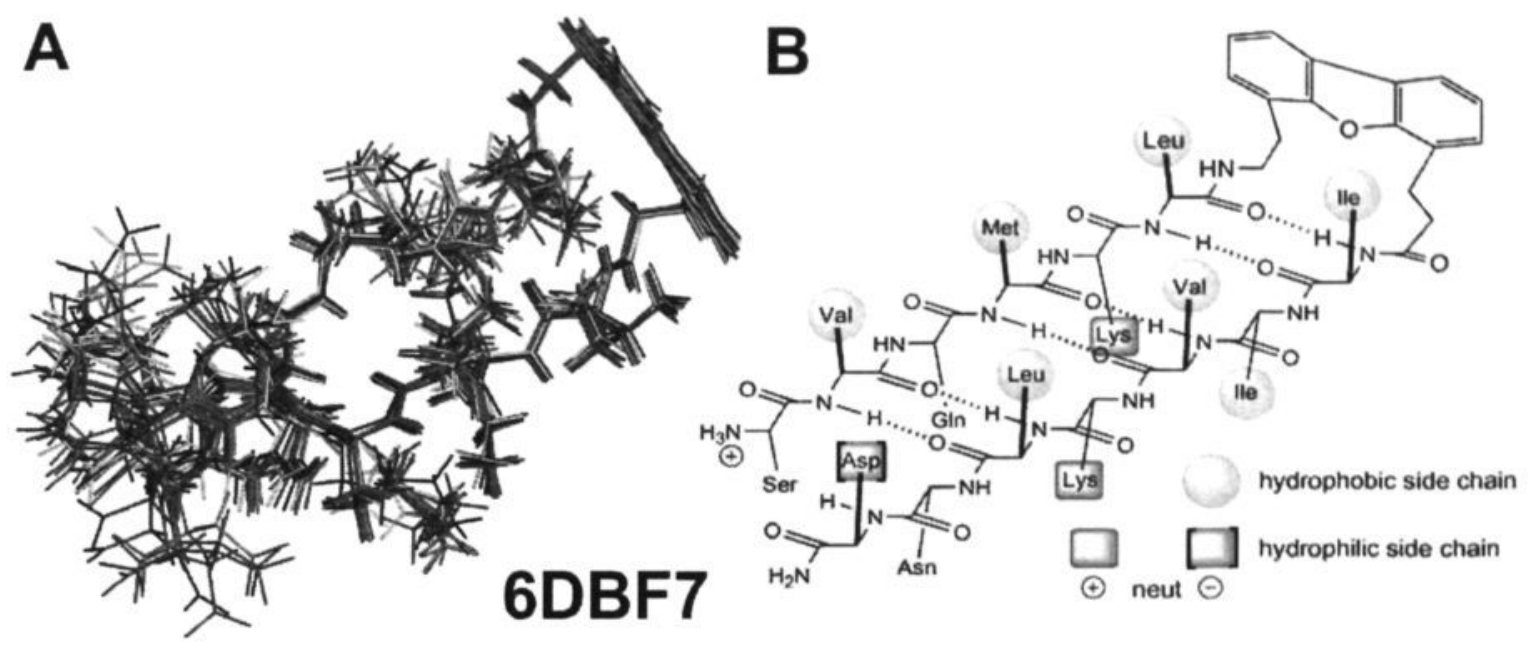

Figure 2. (A) The superposition of 28 structures of 6 DBF7 derived from NMR analysis. The dibenzofuran moiety is shown head-on at the top right of the structures. (B) The chemical 3D structure representation of 6DBF7. Polar residues on the hydrophilic side of the amphipathic Bsheet of 6DBF7 are highlighted with squares, whereas non-polar residues on the hydrophobic side of the amphipathic $B$-sheet of 6DBF7 are highlighted with circles.

\section{DBF analogs retain anti-angiogenic activity}

In endothelial cell (EC) proliferation assays, we demonstrated that 11DBF7, as well as a number of shorter analogs, including 6DBF7, are effective at inhibiting EC growth. Exemplary dose response curves for all XDBF7 analogs are given in Figure $3 \mathrm{~A}$, and $\mathrm{IC}_{50}$ values for all analogs are listed in Table 2. Even though DBF analogs are less active than parent anginex, this may be explained in part by their having a decreased number of residues. Some of the analogs are missing residues identified by alanine scanning as being functionally important (e.g., L5 and 13). Nonetheless, a number of these shorter analogs remain reasonably active, and it appears that the N-terminal hexapeptide SVQMKL and C-terminal hexapeptide IIVKLN are most essential for maintaining anti-angiogenic activity.

Angiostatic potential was further demonstrated in the collagen gel-based sprout formation assay. ${ }^{176}$ Whereas control cultures show numerous sprouts (Figure $3 B$ ), treatment with 6DBF7 (Figure 3C), 11DBF7 (Figure 3D) and anginex (Figure 3E) all demonstrate highly reduced sprouting. These effects are quantified in Table 2 . In general, reducing the number of amino acid residues in the B-strands leads to reduced inhibition of sprout formation, comparable with results from the EC proliferation assay. Nonetheless, some analogs were active in this assay and one of the shortest analogs, 6DBF7, did demonstrate a significant inhibitory effect on tube formation. The kinetics of inhibition using DBF analogs, moreover, were the same as those observed with parent anginex (data not shown). 
Table 2. $\mathrm{N}$ - and $\mathrm{C}$-terminal deletion variants of $11 \mathrm{DBF} 7$ and angiostatic potential.

Amino Acid Sequence $\quad \frac{\text { EC Proliferation }}{\left(\mathrm{IC}_{50}\right)} \%$ Sprouting $^{a}$

Anginex

parent peptide 33mer

$3 \mu \mathrm{M}$

$8 \pm 5 \%$

$\underline{\text { DBF Analogs }}$

\begin{tabular}{|c|c|c|c|}
\hline 11DBF7 & ANIKLSVQMKL-[DBF]-IIVKLND & $12 \mu \mathrm{M}$ & $23 \pm 10 \%$ \\
\hline 10DBF7 & NIKLSVQMKL-[DBF]-IIVKLND & $12 \mu \mathrm{M}$ & $57 \pm 17 \%^{\circ}$ \\
\hline 9DBF7 & IKLSVQMKL-[DBF]-IIVKLND & $20 \mu \mathrm{M}$ & $76 \pm 40 \%$ \\
\hline 6DBF7 & SVQMKL-[DBF]-IIVKLND & $15 \mu \mathrm{M}$ & $60 \pm 12 \%^{\circ}$ \\
\hline 4DBF7 & QMKL-[DBF]-IIVKLND & $25 \mu \mathrm{M}$ & $99 \pm 5 \%$ \\
\hline 3DBF7 & MKL-[DBF]-IIVKLND & $\gg b$ & $84 \pm 30 \%$ \\
\hline 1DBF7 & L-[DBF]-IIVKLND & $\gg b$ & $>b$ \\
\hline 11DBF6 & ANIKLSVQMKL-[DBF]-IIVKLN & $22 \mu \mathrm{M}$ & $115 \pm 7.4 \%$ \\
\hline 11DBF4 & ANIKLSVQMKL-[DBF]-IIVK & $35 \mu \mathrm{M}$ & $115 \pm 16.3 \%$ \\
\hline 11DBF1 & ANIKLSVQMKL-[DBF]-I & $23 \mu \mathrm{M}$ & $111 \pm 13.6 \%$ \\
\hline 6DBF6 & SVQMKL-[DBF]-IIVKLN & $42 \mu \mathrm{M}$ & $96.8 \pm 2.7 \%$ \\
\hline 6DBF4 & SVQMKL-[DBF]-IIVK & $\gg>^{b}$ & $80.4 \pm 0.3 \%$ \\
\hline 6DBF3 & SVQMKL-[DBF]-IIV & $\gg>^{b}$ & $80.0 \pm 17.4 \%$ \\
\hline 6DBF2 & SVQMKL-[DBF]-II & $\gg b$ & $118 \pm 15.8 \%$ \\
\hline 6DBF1 & SVQMKL-[DBF]-I & $76 \mu \mathrm{M}$ & $84 \pm 12.5 \%$ \\
\hline
\end{tabular}

- Significant inhibition $(P<0.03$; Mann-Whitney $U$ test).

"Relative to control culture with medium alone.

${ }^{b}$ The compound had no effect on the assay.

ND, Not Done

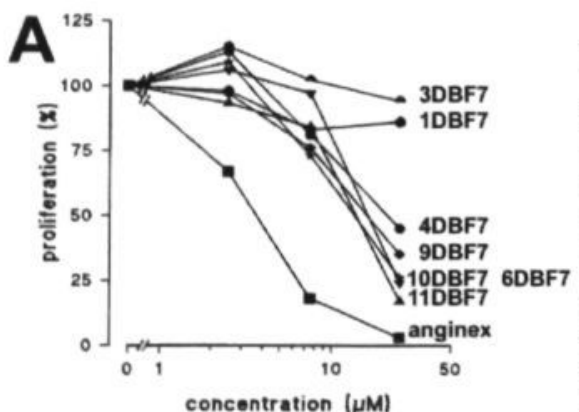

Figure 3. Bioactivity of DBF analogs in in vitro assays. (A) The anti-proliferative effects of some DBF analogs on bFGF-activated EC are plotted as dose response curves. To assess antiangiogenic potential of these DBF analogs, sprouting and tube formation were studied using cytodex -3 beads overgrown with $\mathrm{BCE}$ in a 3-
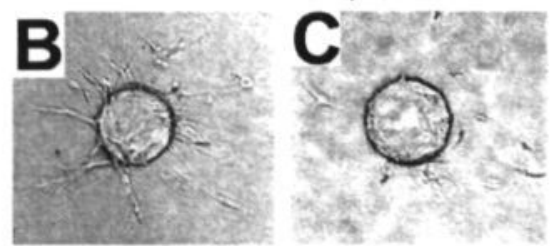
dimensional collagen gel. Tube formation experiments under control conditions (B), or in the presence of 6DBF7 (C), 11DBF7 (D) or anginex (E), at a concentration of $25 \mu \mathrm{M}$ are shown. The beads have

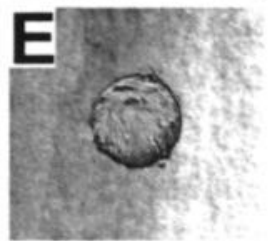
a mean size of $170( \pm 40)$ microns. Using these data, the $\mathrm{IC}_{50}$ on EC proliferation and tube formation were quantified and are listed in Table 2. 


\section{DBF7 and 6DBF7 inhibit tumor growth in mice}

Because our immediate interest in an anti-angiogenic agent is in the area of tumor biology, we assessed the in vivo efficacy of two of the most in vitro active DBF analogs, 11DBF7 and $6 \mathrm{DBF} 7$, in the MA148 xenograft ovarian carcinoma tumor model in athymic mice..$^{154-156}$ Our initial experiments using this model administered the parent analog, 11DBF7, subcutaneously via mini-pumps implanted at the time of inoculation with the tumor cell line. This prevention model demonstrated that treatment of tumor-bearing animals with 11DBF7 resulted in inhibition of tumor growth. Surprisingly, 11DBF7 functioned, on average, slightly better than anginex by reducing tumor volume by up to $80 \%$ relative to tumors from control animals (Figure $4 \mathrm{~A}$ ).

In further experiments, we initiated treatment seven days after inoculation with tumor cells, to allow establishment of tumors prior to the start of treatment. Using this protocol, anginex and $11 \mathrm{DBF} 7$, were found to inhibit tumor growth by up to $70 \%$ during the course of treatment (Figure $4 B$ ). At the end of the four week administration period (day 35), the rate of tumor growth began to increase, but remained at about $50 \%$ ten days post-treatment (data not shown) when animals were sacrificed for analysis of tumor tissue. Interestingly, the smaller analog, 6DBF7, was even more effective than anginex or 11DBF7 at inhibiting the growth of tumors (Figure 4B).

Anti-angiogenic potential in vivo was demonstrated immunohistochemically by staining tumor cross-sections from treated animals with fluorescently-labeled anti-CD31 antibody to identify blood vessels. As shown in Figures 4C-F and quantified in Table 3, vessel density, relative to control (Figure 4C), was significantly reduced by treatment with anginex (Figure 4D), 11DBF7 (Figure 4E) or 6DBF7 (Figure 4F). These anti-angiogenic compounds had a significant effect as well on vessel architecture, demonstrating a drop in the number of end points, branch points and vessel length (Table 3). In addition, anti-angiogenic treatment also reduced the rate of proliferation of tumor cells as determined by immunohistochemical staining of PCNA in cryosections of tumors (Table 3). As a result of angiogenic inhibition, the number of apoptotic tumor cells increased from $311 \pm 103$ in the control, to $620 \pm 146$ and $851 \pm 162$ in anginex and 6DBF7 treated animals, respectively.

In all in vivo experiments, treatment with anginex, 11DBF7 and 6DBF7 did not show any sign of toxicity as assessed by unaltered behavior and normal weight gain during experiments (see inserts to Figure 4A, B). Moreover, hematocrit and creatinine levels in treated animals were normal relative to control, indicating the absence of toxicity to bone marrow and kidney, respectively (data not shown). Upon autopsy, macro- and microscopic morphology of internal organs were also observed to be normal within all experimental groups of animals. 

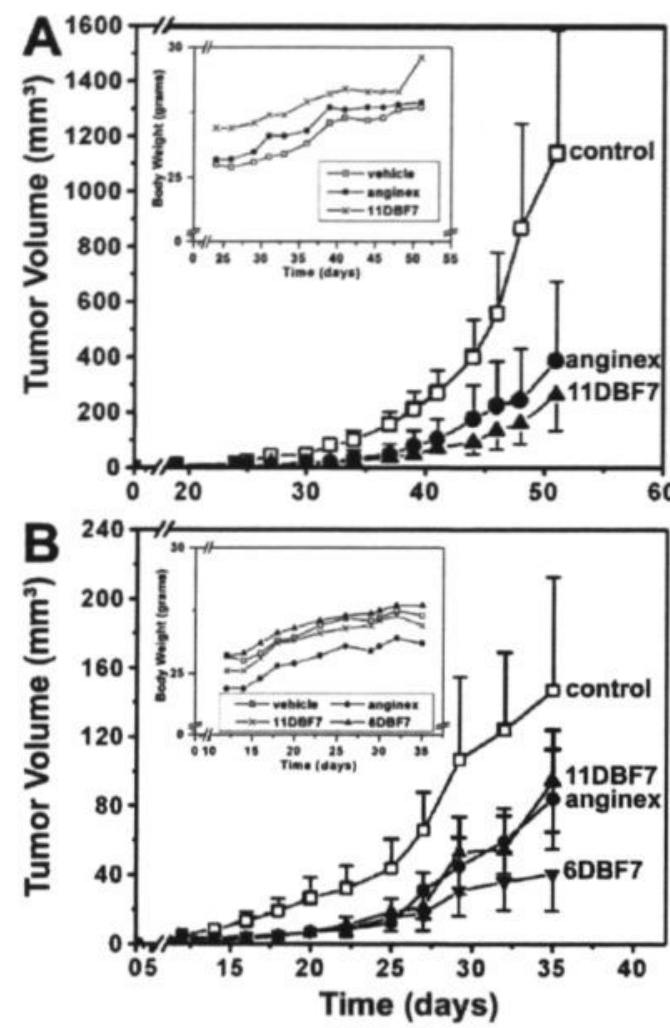

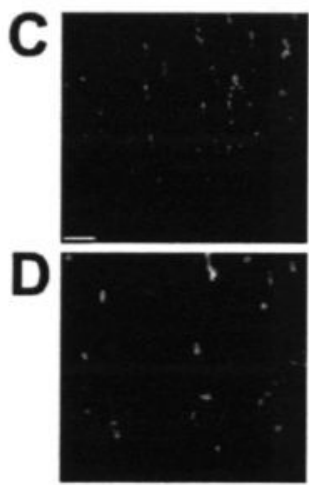

E

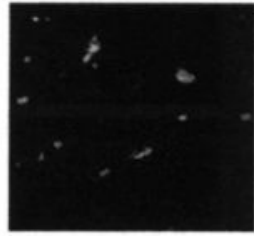

E

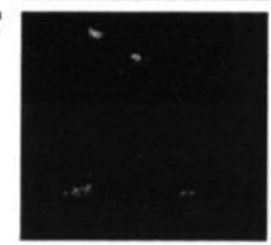

Figure 4. 6DBF7 inhibits tumor growth in mice.

(A) MA148 tumor bearing mice were treated with the optimal dose of anginex (10 $\mathrm{mg} / \mathrm{kg} /$ day) or equivalent doses of 11DBF7 or 6DBF7. Treatment was initiated at the time of inoculation with MA148 cells. (B) An intervention study is shown where tumors were allowed to establish to a palpable size before treatment was initiated. In either study, control groups of animals were treated with PBS containing human serum albumin to control for protein content. Tumor volumes in A and $\mathrm{B}$ are plotted as $\mathrm{mm}^{3}$ $( \pm$ SEM) vs. days post-inoculation (all groups $n=11$ ). The inserts show the body weight development of the mice during the study as a measurement of overall toxicity.

Immunohistochemical analyses of microvessel density is shown ( $\mathrm{C}$ through $\mathrm{F}$ ). $\mathrm{C}$ represents the average amount of microvessels in the tumor of a control treated animal. D, E, and F represent the average amount of vessel density staining on tumor tissue from anginex-, 11DBF7- and 6DBF7-treated animals, respectively. Original magnification $\mathrm{X} 200$; scale bar $=50$ microns.

Table 3. Microvessel density and proliferation rate in tumors of treated mice.

\begin{tabular}{l|c|cccc}
\hline & Proliferation & $\begin{array}{c}\text { Vessel } \\
\text { Density }\end{array}$ & $\begin{array}{c}\text { End } \\
\text { Points }^{\text {c }}\end{array}$ & $\begin{array}{c}\text { Branch } \\
\text { Points }^{\text {d }}\end{array}$ & $\begin{array}{c}\text { Vessel } \\
\text { Length }^{\mathbf{e}}\end{array}$ \\
\hline Vehicle & $848 \pm 104$ & $5858 \pm 656$ & $26.2 \pm 2.2$ & $7.6 \pm 1.4$ & $5.9 \pm 0.7$ \\
Anginex & $414 \pm 44$ & $2245 \pm 329$ & $22.9 \pm 2.2$ & $2.1 \pm 0.6$ & $0.9 \pm 0.3$ \\
11DBF7 & $553 \pm 75$ & $2879 \pm 385$ & $21.2 \pm 3.3$ & $3.1 \pm 0.9$ & $3.0 \pm 0.6$ \\
6DBF7 & $501 \pm 68$ & $2213 \pm 256$ & $21.7 \pm 2.6$ & $2.3 \pm 1.1$ & $2.5 \pm 0.4$ \\
\hline
\end{tabular}

a After binarization of the images of the PCNA-staining, proliferation was estimated by scoring the total number of white pixels per field.

${ }^{b}$ After binarization of the images of the CD31-staining, microvessel density was estimated by scoring the total number of white pixels per field.

${ }^{c}$ Mean number of vessel end points as determined after skeletonization of the images. ${ }^{112}$

${ }^{\mathrm{d}}$ Mean number of vessel branch points/nodes per image.

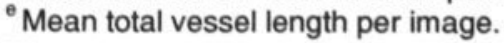

All results are expressed as mean pixel counts per image ( \pm standard error). 


\section{Discussion}

Here, we identified functionally key amino acid residues in the ß-sheet-forming anginex peptide that promote its angiostatic activity and using this SAR information, designed partial non-peptidic B-sheet mimetics of anginex. Members of this novel class of DBF-based compounds are effective anti-angiogenic agents both in vitro and in vivo. Moreover, the in vivo antitumor activity of one of these DBF mimetics, $6 \mathrm{DBF} 7$, is significantly improved over that of the larger parent molecule anginex.

The B-sheet conformation in particular is crucial to the anti-angiogenic activity of these DBF analogs, just as it is for parent anginex peptide. ${ }^{95,176}$ 6DBF7 is shown here to fold as an amphipathic B-sheet, with functionally key hydrophobic residues lying on the same face of the molecule. Interestingly, the crystal structure of VEGF complexed with an anti-angiogenic peptide (Flt-1D2) ${ }^{185}$ derived from one of its endothelial cell-specific tyrosine kinase receptors, Flt-1, indicates that functionally key segments of Flt-1D2 are located within its B-sheet domain. Moreover, one of the peptides derived from this domain, NITVTLKKFPL, inhibits angiogenesis through a non-VEGF binding mechanism, i.e., without binding to VEGF or inhibiting VEGF's binding to its receptors. ${ }^{186} \mathrm{CD}$ and NMR analyses indicate that this Flt-1 peptide 11 mer forms a stable extended $\mathrm{B}$-strand structure in solution. In this conformation, the peptide would present $\mathrm{N}$-terminal hydrophobic amino acid residues I-V-L on the same face of the strand. This I-V-L presentation pattern is also present in 6DBF7 (ß-strand 2), along with the cross-strand hydrophobic pattern V$M-L$ (B-strand 1). Although the efficacy of the Flt-1 peptide is much less than that of $6 D B F 7$, this structural/compositional similarity between these two peptides suggests that on the molecular level, the $\beta$-sheet structure of these molecules is pivotal to their activity ${ }^{187}$ which may suggest signaling through a common cellular receptor.

Many other antiangiogenic proteins whose high-resolution molecular structures are known, e.gs., endostatin ${ }^{47}, \mathrm{PF} 4^{172}, \mathrm{TNF}-\alpha^{79}, \mathrm{BPI}^{52}$, and TSP-1 type 1 repeat ${ }^{51}$, are also structurally (antiparallel $\mathrm{B}$-sheet) and compositionally (high incidence of hydrophobic and positively charged residues) similar to anginex and DBF mimetics. Although this observation may indicate an evolutionary structural link among a number of anti-angiogenic proteins, the meaning of this commonality remains unclear because their mechanisms of action and cellular receptors could be different. To complicate matters further, few receptors reported to mediate the signal leading to angiostasis are definitive and a number of anti-angiogenic agents apparently can act on multiple receptors. For angiostatin, for example, three receptors on endothelial cells have been reported: ATP-synthase ${ }^{39}$ and integrins $\alpha_{v} \beta_{3}$ and $\alpha_{9} \beta_{1}{ }_{1}^{38}$. For endostatin, there are currently also at least three possibilities: a low affinity cell surface glypican receptor, along with a yet-to-be-identified high affinity receptor ${ }^{142}$ and an intercellular epitope (hTM3) in tropomyosin ${ }^{188}$. And for another well-known anti-angiogenic protein, TSP-1, there are four identified receptors: syndecan, heparin sulfate proteoglycans (HSPG), integrin-associated protein (IAP) (reviewed by Chen et al. ${ }^{189}$ ) and its 
long-known transmembrane receptor CD36 $6^{190,143 .}$

Even with these uncertainities, the compositional and structural similarity noted among anginex, $6 \mathrm{DBF} 7$ and various anti-angiogenic proteins and peptides may be used to further improve or optimize the efficacy of anginex peptide mimetics and in the design of additional novel therapeutic anti-angiogenic agents. For the present, 6DBF7 appears to be one of the most potent anti-angiogenic and anti-tumor agents known. This claim is supported by the observation that anginex is as potent as angiostatin and more potent than endostatin at inhibiting the growth of ovarian tumors in mice. ${ }^{155}$ Therefore, based on the present comparison with anginex, 6DBF7 must also be more potent in vivo than endostatin or angiostatin. The design of 6DBF7 places us on the road to creating an actual small-molecule pharmaceutical agent that could be used as an effective therapeutic against cancer, as well as against other angiogenically-related pathologic disorders like arthritis, restenosis, endometriosis and diabetic retinopathy. 


\section{Chapter 7}

\section{General discussion}

Partly published in Angiogenesis. 2003; 5(4): 1-12. 


\section{General discussion}

The lack of major clinical breakthroughs underscores the need to identify and develop more and better angiogenesis inhibitors. Accordingly, the aim of this thesis was to test and optimize the cancer inhibiting capacity of anginex, and to develop new angiogenic inhibitors with de novo peptide design.

The research described in this thesis shows for the first time that it is possible to generate angiostatic molecules by peptide design. It is shown that anginex has anti-angiogenesis activity in vitro and that this angiostatic property can be used to obtain a profound anti-cancer effect in vivo in preclinical models. The mechanism of action of anginex was found to be the induction of anoikis leading to apoptosis in angiogenically activated endothelial cells. ${ }^{36}$ This resulted in an up to $90 \%$ inhibition of migration in the wound assay and significant inhibition of angiogenesis as demonstrated in the in vitro mouse aortic ring assay. In addition, tumor-induced angiogenesis in the chick chorioallantoic membrane was markedly inhibited. Anginex showed profound anti-tumor activity in the syngeneic mouse B16F10 melanoma model and in a xenograft human tumor model MA148. In the MA148 model, tumor growth was inhibited dose-dependently by up to $80 \%$ when systemically administered via osmotic mini-pumps starting at the time of tumor cell inoculation. When anginex was conjugated to human serum albumin, effectiveness was significantly improved, most likely due to the increased bioavailability of the conjugate.

Currently, the clinical effect of angiogenesis inhibitors can not be accurately evaluated due to (i) the lack of relevant clinical assays and (ii) the sparseness of reliable surrogate endpoints. At least, when the inhibitors are used as monotherapies, the absence of tumor regression (objective response) makes evaluation of the potential efficacy of such a drug in phase I and phase II a difficult problem. ${ }^{191}$ Until a clinical angiogenesis assay is established and validated, or a surrogate molecular tumor angiogenesis marker has been developed, determining the optimum dose and route of administration of these agents will remain difficult. Furthermore, the complexity of the angiogenic process means that it is unlikely that inhibiting a single pathway will result in significant tumor shrinkage with tumors selecting alternative angiogenic routes once one is blocked. Combinatorial treatment regimes targeting a variety of different angiogenic pathways is likely to be a more successful strategy. However, the total disappearance of tumors was not seen when anginex was combined with another well-known anti-angiogenesis inhibitor angiostatin, although this still resulted in a synergistic effect. This synergy between angiostatin and anginex suggests that their mechansim of action are different and that they can augment each other as anti-angiogenic agents. These findings suggest that combining therapies which target different aspects of the tumor mass, such as the stroma and the parenchyma should be explored. Teicher et al. was among the first who showed that anti-angiogenic agents can potentiate cytotoxic cancer 
therapies. ${ }^{148,192}$ Here we have tested a combination of anginex with the chemotherapeutic carboplatin in a human ovarian xenograft model. Carboplatin is a fist line chemotherapeutic in ovarian cancer and combined with anginex complete disappearance of the tumors was observed.

\section{Mechanisms of action among anti-angiogenics}

Considering several protein fragments, among those listed in chapter 1 , it should be apparent that aside from their functional commonality of being anti-angiogenic, these compounds, for the most part, are also structurally and compositionally similar. The vast majority fold as antiparallel $B$-sheets and contain a relatively high incidence of hydrophobic and cationic residues. As structure-function relationships in other anti-angiogenic proteins and peptides become known, it will be interesting to see if this is a common feature among anti-angiogenic proteins.

In addition, many other anti-angiogenic proteins, e.g., $\mathrm{PF}^{172}, \mathrm{TNF}^{79}$ and $\mathrm{BPI}^{52}$, are also structurally and compositionally similar to protein fragments and smaller peptides discussed prior. Although the significance of this correlation is unclear, it appears that the mechanisms of action of these anti-angiogenic agents are not the same, i.e., they function using different receptors. Few receptors reported to mediate the signal leading to angiostasis have been definitive reported. In fact, the picture in terms of receptors is quite untransparent and a number of these agents apparently can act on multiple receptors. For example, ATP-synthase on endothelial cells has been reported to be a receptor for angiostatin. ${ }^{39} \mathrm{However}$, other angiostatin receptors include $\alpha_{v} \beta_{3}$ and, to a lesser extent, $\alpha_{9} \beta_{1}{ }^{38}$. Karumanchi et al. ${ }^{142}$ demonstrated the existence of a low affinity cell surface glypican receptor for endostatin, along with a yet to be identified high affinity receptor, and a third intercellular protein, an epitope (hTM3) in tropomyosin, was also found to bind endostatin. ${ }^{188}$ Another such example is TSP-1, which requires, both in vitro ${ }^{190}$ and in vivo ${ }^{143}$, the expression of the transmembrane receptor CD36. However, TSP-1 is also known to bind to other receptors, such as Syndecan, heparin sulfate proteoglycans (HSPG) and integrin associate protein (IAP) (reviewed in Chen et al. ${ }^{189}$ ). The presence of multiple receptors, although possibly being therapeutically beneficial, complicates our understanding of tumor angiogenesis and our ability to develop receptor-specific antagonists.

\section{Identification, design and development of new anti-angiogenic agents}

Identification of anti-angiogenic fragments of proteins that are themselves anti-angiogenic, prompted the search for even smaller anti-angiogenic peptides. ${ }^{93}$ This search was motivated by a desire to minimize the therapeutic dosage, to produce the peptide synthetically rather than by using recombinant techniques, to help identify an orally active agent, and, quite simply, to generate new improved anti-angiogenic compounds. Several approaches were used to achieve this goal: peptide dissection, phage-display, and peptide design. ${ }^{193}$

Peptide dissection is most commonly for relatively large endogenous molecules that either have great toxicity or lack specificity. For example, the use of intact TSP-1 as an anti-angiogenic 
drug in humans is not practical due to its size $(450 \mathrm{kDa})$ and not desirable due to its diverse and multiple biological activities. For example, TSP-1 is involved in neurite outgrowth, platelet aggregation, as well as angiogenesis. ${ }^{194} \mathrm{However}$, anti-angiogenic peptides derived from native TSP-1 could provide a reasonable alternative. Small peptides were generated and tested (e.g. Mal II). Unfortunately, micromolar concentrations of these peptides were required to achieve an effect equivalent to that produced by low nanomolar amounts of native TSP-1. This loss of activity could be recovered in a 7 mer by substituting an L-amino acid with its $\mathrm{D}$-isoleucyl enantiomer and even optimized by ethylamide-capping. ${ }^{194}$ Following further modification to a nonamer containing the non-natural amino acid norvaline (see Table 2), this peptide successfully inhibited tumor growth. ${ }^{195}$ Similar strategies of peptide dissection have been applied for, tumstatin ( $\left.\alpha 3(\mathrm{IV}) \mathrm{NC} 1\right)$ and its deletion mutant tum- 5 (residues $54-132)^{196}$, and parathyroid hormone-related peptide $(\mathrm{PTHrP})^{197}$.

Table 1. Sequences of selected anti-angiogenic peptides.

\begin{tabular}{l|lc}
\hline Name & Sequence & Source \\
\hline EGF $_{33-42}$ & RDGSYGIV & 75 \\
Mal II & SPWSSASVTAGDGVITRIR & 194 \\
$7 m e r$ & Ac-G V I* T R I R-Neth & 194 \\
DI-TSP & Ac-G V I* T Nva I R P-Neth & 195 \\
PTHrP & AVSEHQLLHD & 197 \\
T3 & LQRFTTMPFLFCNVNDVCNF & 196 \\
T7 & TMPFLFCNVNDVCNFASRNDYSYWL & 196 \\
RGD-4C & CDCRGDCFC & 198 \\
NGR-4C & CNGRCVSGCAGRC & 198 \\
FIt2-11 & NITVTLKKFPL & 186 \\
Anginex & ANIKLSVQMKLFKRHLKWKIIVKLNDGRELSLD & 36 \\
\hline
\end{tabular}

* the L-amino acid is substituted by the D-enantiomer

Phage display technology has been used to isolate peptides that specifically home to angiogenic (tumor) blood vessels. This revealed a number of peptide motifs that specifically bind to tumor vasculature. One of these peptides, RGD-4C $\left\{\mathrm{d}(\mathrm{CDCRGDCFC})_{2}\right\}$ is a molecule containing the integrin-binding sequence RGD (Arg-Gly-Asp; Table 1). ${ }^{198-200}$ Another peptide, $\mathrm{d}\left(\mathrm{CNGRCVSGCAGRC)}\right.$, with a $\mathrm{CX}_{3} \mathrm{CX}_{3} \mathrm{CX}_{3}$ structural motif contains the sequence NGR (AsnGly-Arg; Table 1), known to be another cell adhesion binding motif. Phages expressing these peptides specifically home in on angiogenic blood vessels from different tumor types, including carcinomas, sarcomas, melanomas. A third peptide containing the motif GSL (Gly-Ser-Leu) is 
also frequently recovered from breast carcinoma and Karposi's sarcoma. ${ }^{198}$ Phage display has also been used to identify peptides that bind to VEGF and thereby inhibit its angiogenic activity. By using this approach, Fairbrother et al. ${ }^{201}$ identified a number of VEGF-binding peptides, and Pan et al. ${ }^{202}$ reported the NMR structure of one of these peptides, a 19 mer. This peptide has a turn-helix conformation with hydrophobic residues partitioned to one face of the folded peptide and polar/charged residues at the other face. Binding to the VEGF dimer is promoted via interactions with hydrophobic residues.

Peptide design was applied in order to generate peptides that would interfere with the binding of VEGF and FIt-1. VEGF functions through two endothelial specific tyrosine kinase receptors, Flt-1 (VEGFR-1) and Flk-1 (VEGFR-2). Tan et al. (2001) demonstrated that an 11 amino acid peptide (Flt2-11; Table 1) derived from the second immunoglobulin domain of Flt-1 functions as an angiogenesis inhibitor by inhibiting VEGF function through a non-VEGF binding mechanism, i.e., without binding to VEGF or inhibiting VEGF's binding to its receptors. ${ }^{186}$ Peptide sequences in Flt-1 were identified primarily using Flt-1 VEGF-binding domain data by mutational analysis ${ }^{203}$ and the VEGF-FIt-1D2 protein crystal structure. ${ }^{185}$ Of the two peptides chosen (SPNITVTLKKFPL and RPFVEMYSEIPE), the former one demonstrated anti-angiogenic activity in the CAM assay, and this activity could be slightly enhanced by removing the $\mathrm{N}$-terminal dipeptide SP.

Rational peptide design was the strategy our laboratories applied in order to generate new angiogenesis inhibitors derived from anginex. Since residues $L 5$, V7, K10, K17, 120, V22, L24 in anginex were identified as being crucial to anti-proliferative activity, the C-terminus was not deemed to be essential for activity and therefore was omitted in the design of a series of peptides. Furthermore, it was noted that in the $B$-sheet conformation all five hydrophobic residues are proximal and are conformed on the hydrophobic face of the amphipathic $\beta$-sheet. ${ }^{95,193}$ We designed the DBF-series of partial-peptide mimetics, in which $B$-strand 3 and turn 2 of anginex were omitted and a dibenzofuran (DBF) B-turn mimetic ${ }^{183,184}$ was used in place of turn 1 and the remainder of $B$-strand 1 (residues 1-11) and B-strand 2 (residues 20-26). The DBF B-turn mimetic was used in order to maintain the bioactive $\beta$-sheet conformation of anginex. ${ }^{193}$ 


\section{Future directions}

Besides combining anti-angiogenic therapy with conventional therapy, angiogenesis inhibitors are now being used as 'homing'-devices for chemotherapeutics in the hope of increasing specificity and consequently reducing toxicity. The tumor-homing capacity of EC-specific peptides was successfully used to target doxorubicin, a frequently used chemotherapeutic with intrinsic anti-angiogenic activity. ${ }^{198} \mathrm{In}$ xenograft models, this targeting approach resulted in prolonged survival, reduction of the optimal effective dose and reduced toxicity. Targeting can be done either classically by linking the chemotherapeutic chemically to the anti-angiogenic compound or in a novel approach by linking it to peptides which specifically target tumor vasculature ${ }^{199}$.

Mechanistically many angiogenesis inhibitors have multiple receptors and their precise mechanism of action has not been elucidated for most of them. This entails the possibility of a cross-reaction with other different physiological processes, for example as seen with TSP-1. ${ }^{189}$ Finding out the precise mechanisms and the possible interactions with, for example, the immune system and the coagulation system is going to be very intriguing and an essential part of their future clinical development. Moreover, some anti-angiogenesis compounds (anginex, PF-4 and BPI) also display bactericidal capacity. Structural function relationship analysis has to elicit whether the anti-angiogenesis and anti-bacterial properties are linked within the protein and whether this is a general commonality among angiogenesis inhibitors. For anti-angiogenic proteins, structure-activity relationships, i.e., specific residues and their relative conformations, which impart activity, are sorely needed, and even the analysis of high-resolution molecular structures of a number of anti-angiogenic proteins, e.g., endostatin ${ }^{47}, \mathrm{PF}^{172}$ and $\mathrm{BPI}{ }^{52}$, has not provided this information. Working with a small peptide like anginex, however, allows relatively easy assessment of structure-activity relationships.

The compositional and structural similarity noted among these anti-angiogenic peptides may be used, possibly via combinatorial approaches, to design additional therapeutic anti-angiogenic agents. In the context of a B-strand conformation, for example, the Flt-1 peptide NITVTLKKFPL would present N-terminal hydrophobic amino acid residues I-V-L on the same face of the strand. This presentation pattern is observed in a number of anti-angiogenic peptides. In fact, anginex shows the same B-strand I-V-L pattern, as well as the cross-strand pattern, V-M-L. Because anginex, for example, forms $\beta$-sheet structure and can be used as a presentation scaffold, it might be possible to vary a set number of residues and make analogs having various permutations and combinations of amino acid residues. In this way, one might optimize angiostatic potential. In addition, it might be possible to reduce the size of the active peptide, which could help lead to the design of an actual small-molecule mimetic of an anti-angiogenic peptide. 


\section{Conclusions}

Anginex is an effective angiogenesis inhibitor in vitro as well as in vivo. It's activity is most likely due to the induction of anoikis and the inhibition of endothelial cell migration anginex possesses anti-tumor capacity in several mouse models. When combined with conventional treatment anginex is able to cause complete disappearance of tumors. The complete absence of toxicity which was observed in all the mouse models used, demonstrated that anginex is a promising agent for further clinical development.

After identifying key amino acid residues that promote anginex' angiostatic activity, a peptide mimetic was designed carrying only 13 of the original 33 amino acid residues. This new generated compound showed improved anti-tumor activity. Both the significant reduction in the number of residues required to promote angiostatic activity and the use of an organic B-sheetinducing scaffold places us on the road to creating an orally-active, small-molecule mimetic of an anti-angiogenic protein. Oral administration allows the continuous exposure of the tumor to the inhibitor over prolonged periods of time which may be the optimum means of inhibiting angiogenesis in vivo thus turning cancer into a chronic disease which does not kill the patient. 
Chapter 8

Summary / Samenvatting 


\section{Summary}

The process of generating new blood vessels from pre-existing vasculature is termed angiogenesis. Angiogenesis takes place in several developmental stages and multiple physiological and pathological processes. One of those pathological processes is cancer. Chapter 1 gives a general introduction to the field of cancer related angiogenesis and to the plethora of molecules involved in it. Until now many of angiogenesis inhibitors have been derived from endogenous origins. However, the emphasis of this thesis lies on the design and development of new exogenous angiogenesis inhibitors. These inhibitors were designed using a combinational approach employing basic folding principles and incorporating short sequences from $\beta$-sheet domains. This approach generated a library of peptides that were tested for their anti-angiogenic capacity. One peptide shown to be most potent, $\beta$-pep25, was named anginex. Anginex is a 33mer whose structure was based on the 3D structure of $\alpha$-chemokines such as platelet factor4 and interleukin- 8 and the $\beta$-sheet domain containing molecule bactericidal/ permeability-increasing protein (BPI). Chapter 2 describes that the anti-angiogenic capacity of anginex is also present in vivo and that anginex can inhibit tumor growth in a human ovarian carcinoma xenograft model. Various treatment regimes, the mode and frequency of administration, as well as dose response curves, were investigated and continuous systemic treatment in a $10 \mathrm{mg} / \mathrm{kg} /$ day dose provided optimal efficacy. This was improved by conjugating anginex to human serum albumin to enhance its bioavailability, which led to $75 \%$ tumor growth inhibition even 30 days post-treatment. Chapter 3 shows that besides the human ovarian xenograft model anginex also entails anti-tumor activity in a murine syngeneic carcinoma model (B16F10 melanoma in C57BL 6 mice). The absence of toxicity in all of the models used, indicates that anginex is a very promising anti-cancer agent that should be developed for clinical testing. The mechanistic studies (both in vitro as well as in vivo) described in chapter 3 showed that anginex exerts its antiangiogenesis effect by specifically targeting tumor endothelial cells.

Besides testing anginex as a mono-therapeutic agent, it was also investigated in combination with conventional chemotherapy or the well-known angiogenesis inhibitor angiostatin. As described is chapter 4 , the combination of anginex with a sub-optimal dose of chemotherapeutic carboplatin resulted in tumor remissions to microscopic disease. Treatment in combination with angiostatin demonstrated a synergistic effect at inhibiting tumor growth. Immunohistochemical assessment of the microvessel density indicated that this tumor growth inhibition by anginex is explained by its anti-angiogenic activity. In addition, an increase in apoptotic cells and a decrease in proliferating cells was noticed in anginex treated tumors compared to vehicle treated tumors.

By generating a new series of anginex related molecules the structural bioactive conformation of anginex was investigated, which is described in chapter 5 . This series of molecules were modified by excluding 6 amino acids of the $\mathrm{C}$-terminus of anginex and by introducing disul- 
fide bonds. These covalent constraints limited conformational space available to the peptide, such that, for example, helix formation could not occur. As long as placement of disulfide bonds preserved the $B$-strand alignment as in the proposed bioactive conformation, activity was preserved.

Based on further structure-activity relationships of these $B$-sheet-forming peptides, partial mimetics were designed. In order to maintain the $\beta$-sheet conformation a dibenzofuran turnmimetic was introduced, as described in chapter 6 . One of these novel anti-angiogenic molecules, 6DBF7, was more effective in vivo than parent anginex. In a mouse xenograft model for ovarian carcinoma, 6DBF7 was observed to reduce tumor growth by up to $80 \%$. Because in vitro tube formation was inhibited by $6 \mathrm{DBF} 7$ and treatment of tumor-bearing mice resulted in a significant reduction in microvessel density within the tumor, it was suggested that the mechanism of $6 D B F$ is also based on angiogenesis inhibition. This partial peptide-mimetic is the first molecule designed as a substitute for an angiogenesis inhibitory agent.

Chapter 7 reviews the current anti-angiogenesis peptides and their diversity in methods of identification. In addition, commonalities were addressed in this chapter, followed by possible future directions. 


\section{Samenvatting}

De vorming van nieuwe bloedvaten uit reeds bestaande bloedvaten in het lichaam noemt men angiogenese. Naast een belangrijke rol in de embryonale fase, speelt angiogenese een essentiële rol tijdens fysiologische en pathologische processen. Een voorbeeld van zo'n pathologische conditie is kanker. Een algemene introductie over kankergeassocieerde angiogenese staat beschreven in hoofdstuk 1. Angiogeneseremming biedt een unieke kans om tumorgroei tegen te gaan en is gebaseerd op andere principes dan de traditionele behandelingsmethoden zoals chirurgie, bestraling en chemotherapie. Anti-angiogenese therapie beïnvloedt de bloedvaten in tegenstelling tot de conventionele therapieen die meestal gericht zijn tegen de tumorcellen zelf. De meeste tot nu toe beschreven angiogeneseremmers zijn van endogene afkomst. Dit proefschrift beschrijt de ontwikkeling van specifieke exogene remmers. Deze remmers werden gecreëerd aan de hand van de 3-dimensionale structuur van $\beta$-sheet bevattende cytokines zoals plaatjesfactor- 4 en interleukine- 8 . Tevens hebben deze 33 aminozuren bevattende peptiden $\beta$-sheet gedeeltes van bactericidal/permeability-increasing protein (BPI). $\mathrm{Bij}$ deze toepassing is een bibliotheek van peptiden gegenereerd, welke getest werd op antiangiogene activiteit. Eén van deze peptiden, Bpep-25, bleek de grootste in vitro angiogenese remmende activiteit te bezitten en werd geselecteerd voor verdere ontwikkeling. $\beta$ pep- $25 \mathrm{kreeg}$ de naam anginex.

Hoofdstuk 2 beschrijft dat deze anti-angiogene werking van anginex ook in vivo wordt waargenomen en dat deze werking de remming van tumorgroei kan bewerkstelligen. Verschillende doseringen en behandelschema's werden onderzocht. Een continue systemische behandeling met een concentratie van $10 \mathrm{mg} / \mathrm{kg} /$ dag resulteerde in een optimaal effect. Dit werd waargenomen in een humaan ovariumcarcinoom model in naakte muizen. De tumorgroeiremming bleek vergroot te kunnen worden door anginex te koppelen aan humaan serum albumine. Dit leverde een inhibitie op van $75 \%$ tot zelfs 30 dagen na het einde van de behandeling. Hoofdstuk 3 laat zien dat naast een goede werking in het ovariumcarcinoom model, anginex ook in staat is tumorgroei te remmen in een syngeen muismodel (B16F10 melanoom in C57BL/6 muizen). Het uitblijven van enige vorm van toxiciteit in alle geteste muismodellen, suggereert dat anginex een veelbelovend medicijn tegen kanker kan worden. De mechanistische studies (in vitro en in vivo) in hoofdstuk 3 laten zien dat anginex specifiek werkt via het endotheel van de tumoren.

Naast het testen van anginex als monotherapie zijn er ook combinatietherapieën uitgevoerd. Hoofdstuk 4 beschrijft de combinatie van anginex met traditionele chemotherapie en een andere bekende angiogeneseremmer angiostatine. De combinatie behandeling met chemotherapeutica, zoals de platina-verbinding carboplatin, liet een complete remissie van de tumoren zien. De combinatie therapie met angiostatine liet een synergistisch effect zien met betrekking tot het remmen van tumorgroei. De tumorgroeiremming werd verklaard door een vermindering van het aantal capillaire bloedvaten in de tumor. Dit werd aangetoond met behulp 
van immunohistochemische experimenten. Deze proeven lieten tevens zien dat er een verhoogd aantal cellen apoptose onderging en een verlaagd aantal prolifererende cellen aanwezig was in tumoren die behandeld waren met anginex, in vergelijking met controle tumoren.

De bio-actieve structuur van anginex werd onderzocht met behulp van de nieuwontwikkelde anginex gerelateerde moleculen die beschreven staan in hoofdstuk 5 . Een nieuwe serie peptiden werd gesynthetiseerd waarbij zes $C$-terminale aminozuren werden verwijderd en zwavelbruggen werden geïntroduceerd om de vouwvrijheid van het peptide te belemmeren. Indien de plaats van een zwavelbrug de $B$-sheet structuur waarborgde - de veronderstelde bioactieve conformatie - bleef de anti-angiogene activiteit behouden.

Aan de hand van additionele structuur-activiteit proeven met anginex werden gedeeltelijke mimetics ontworpen. Om de bio-actieve $\beta$-sheet structuur te handhaven, werd een dibenzofurangroep (DBF) geïntroduceerd. Deze dibenzofuran-groep is beschreven als 'turn-mimetic'. Deze studies staan vermeld in hoofdstuk 6 . Eén van deze gegenereerde moleculen, 6 DBF7, was in in vivo tumor modellen effectiever dan anginex. In het hiervoor genoemde humaan ovariumcarinoom muismodel was $6 \mathrm{DBF} 7$ in staat om $80 \%$ van de tumorgroei te remmen. Als gevolg van toediening van $6 \mathrm{DBF} 7$ werd een vermindering in de in vitro spruitformatie waargenomen en bleek de microvaatdichtheid in muizentumoren sterk afgenomen. Dit suggereert dat angiogenese remming ook ten grond slag ligt aan de tumorgroeiremming van 6DBF7. Deze 'partial mimetic' is de eerst rationeel ontworpen substitutie voor een angiogene remmer. $6 \mathrm{DBF} 7$ is maar half zo groot als anginex en met een behouden dan niet vermeerderde tumorremmende activiteit, is dit een hoopvolle ontwikkeling op de weg naar een 'small-molecule' angiogeneseremmer.

Een samenvatting van de enkele nu bekende angiogenese remmers staan beschreven in hoofdstuk 7. In dit hoofdstuk worden de overeenkomsten van de verschillende remmers besproken en de toekomstverwachtingen in het kanker gerelateerde angiogeneseonderzoek. Tevens worden in dit hoofstuk de nieuwe technieken vermeld die gebruikt worden om meer en betere angiogenese remmers te ontdekken en verder te ontwikkelen, in de hoop een nieuwe krachtige behandelmethodiek te ontwikkelen naast de reeds bestaande geaccepteerde therapieën. 


\section{Acknowledgements / Dankwoord}

Let it be clear that the thesis you have before you is by no means a one man accomplishment. So some words of thanks are in order.

I would like to thank my trinity of science: Prof. Dr. A.W. Griffioen, Prof. Dr. K.H. Mayo and Prof. Dr. S. Ramakrishnan for their guidance and motivation, as well as for sharing with me their individual vision of science and research.

I thank Judy Haseman for being the heart and soul of the lab and the backbone of my research.

Also I want to thank my past and present colleagues. I hope you have learned as much from me, as I have from you on both scientific and personal level. Especially I would like to thank Monica Arroyo, Nathan Lockwood and Irina Nesmelova for their structural and compositional input displayed in this thesis, and Prof. Dr. T. Hoye and his lab for co-developing the peptides. I thank Dinesha Walek and the Microchemical Facility for peptide synthesis, as well as Loes van Eijk and Daisy van der Schaft for their additional input regarding the in vitro angiogenesis assays.

I would like to say thanks to Prof. Dr. J. Wagstaff for giving me this opportunity and my paranimphen Robert van Tankeren en Victor Thijssen for standing by me and believing in me.

But of course also many thanks to the people who were always there for me, my family and my friends, both Dutch and International. Bao I thank you for past, present and hopefully future.

Ich bedank Marieke um mich onbewus mien naas de gooije kangk op te laote wieze, en mien 'blood gezjwaore vrinde' (blood en liters beer)': de Ridders van de Rông Taofel met eure Camelot de Casa Loco.

Maar netuurlijk auch mien femilie: mien mooder um nog sjteeds altied klaor te sjtaon veur mich en mien vrinde, euver onverwaardelikke leefde gesjpraoke, en mien zus um mich toe te huure en beej te sjteure, misschien waal mier den det zeej oëts zoel door hebbe. En mien vader um mich nog sjteeds achter de vaere te zitte.....

Minse Merci,

Saluté

People thanks for the diversity and most of all thanks for sharing that,

Ruud,

May 2003, Minneapolis/Saint Paul 


\section{Curriculum Vitae}

Name: $\quad$ Ruud Petrus Maria Dings

Date of birth: $\quad$ August $6^{\text {th }}, 1977$

Place of birth: $\quad$ Tegelen, The Netherlands

Sex: $\quad$ Male

Nationality: Dutch

Education

1989-1995

1995-1996

1998

1999

1996-2000

2000
Collegium Marianum, Venlo, The Netherlands (High School).

B.Sc. Health Sciences, University of Maastricht, The Netherlands.

Course for registration as a radiology worker.

Interfaculty Reactor Institute, Delft, The Netherlands.

Course for registration as a laboratory animals usage worker.

Central Animal Facility, University of Maastricht, The Netherlands.

M.Sc.-student Biological Health Sciences, University of Maastricht. M.Sc.-degree Biological Health Sciences.

\section{Professional Experience:}

1999 Major subject:

Experimental Oncology- The regulation of the expression of ICAM-1 on endothelial cells during angiogenesis and intervention by specific stimulators and inhibitors.

Tumor Angiogenesis Laboratory, Department of Internal Medicine, University Hospital Maastricht (supervised by Prof. Dr. H.F.P. Hillen and Prof. Dr. A.W. Griffioen).

1999 Six month trainee-ship abroad

Angiogenesis inhibitors overcome tumor endothelial cell anergy.

Department of Pharmacology, University of Minnesota, Minneapolis, MN, U.S.A. (supervision Prof. Dr. S. Ramakrishnan)

2000-present Testing and optimalizing the in vivo anti-angiogenic properties of the novel angiogenesis inhibitor anginex.

Ph.D.-student affiliated to University of Minnesota (Dept. of Biochemistry, Molecular Biology and Biophysics; supervision Prof. Dr. K. H. Mayo) \& University Hospital Maastricht (Depts. Internal Medicine / Pathology; supervision Prof. Dr. A.W. Griffioen). 


\section{List of publications}

\section{Papers}

Dings, R. P.M., Yokoyama, Y., Ramakrishnan, S., Griffioen, A. W., and Mayo, K. H. The designed angiostatic peptide anginex synergistically improves chemotherapy and antiangiogenesis therapy with angiostatin. Cancer Res, 63.382-5, 2003.

Dings, R. P. M., Arroyo, M. M., Lockwood, N. A., Van Eijk, L. I., Haseman, J. R., Griffioen, A. W., and Mayo, K. H. B-sheet is the bioactive conformation of the antiangiogenic anginex peptide. Biochem J, 373:1-8, 2003.

Dings, R. P. M., Nesmelova, I., Griffioen, A. W., and Mayo, K. H. Discovery and development of anti-angiogenic peptides: a structural link. Angiogenesis, 5:1-12, 2003.

Dings, R. P. M., van der Schaft, D. W., Hargittai, B., Haseman, J. R., Griffioen, A. W., and Mayo, K. H. Anti-tumor activity of the novel angiogenesis inhibitor anginex. Can Lett, 194:55-66, 2003.

van der Schaft, D. W., Dings, R. P.M., de Lussanet, Q. G., van Eijk, L. I., Nap, A. W., Beets-Tan, R. G., Bouma-Ter Steege, J. C., Wagstaff, J., Mayo, K. H., and Griffioen, A. W. The designer anti-angiogenic peptide anginex targets tumor endothelial cells and inhibits tumor growth in animal models. Faseb J, 16:1991-3, 2002.

Tromp, S. C., oude Egbrink, M. G., Dings, R. P.M., van Velzen, S., Slaaf, D. W., Hillen, H. F., Tangelder, G J., Reneman, R. S., and Griffioen, A. W. Tumor angiogenesis factors reduce leukocyte adhesion in vivo. Int Immunol, 12:671-6, 2000.

Mayo, K.H., Dings, R.P.M., Flader, C., Nesmelova, I., Hargittai, B., van der Schaft, W.J., van Eijk, L.I., Walek, D., Haseman,J.R., Hoye, T.R., and Griffioen, A.W. Design of a partial-peptide mimetic of anginex with antiangiogenic and anticancer activity. submitted.

Dings, R.P.M., Williams, B.W., Griffioen, A.W., Mayo. K.H, Griffin, R. Anginex synergistically improves tumor radiation response and suppresses tumor growth in multiple animal models. in final preparation.

Wild, R., Dings, R.P.M., Ramakrishnan S. Carboplatin selectively induces the VEGF stress response in endothelial cells: synergistic inhibition of tumor growth by combina tion treatment with antibody to VEGF. submitted.

Wild, R., Yokoyama, Y., Dings, R.P.M., Ramakrishnan S. VEGF-Toxin conjugate inhibits mammary adenocarcinoma development in a transgenic mouse model of spontaneous tumorgenesis. submitted.

Thijssen, V.L.J.L., Brandwijk, R.J.M.GE., Dings, R.P.M., Griffioen, A.W. Simultaneous molecular profiling of angiogenesis in tumor cells and stromal cells in a xenograft tumor model. submitted. 


\section{List of publications}

Abstracts

Dings, R.P.M., Flader, C., Hargittai, B., Haseman, J., van Eijk, L., Hoye, T., Griffioen, A.W., Mayo, K.H. On the road to a small molecule mimetic of the peptide anginex, a potent anti-angiogenic, anti-tumor agent. Proceedings of the AACR, 2002, abstract.

Dings, R.P.M., Yokoyama, Y., Ramakrishnan, S., Sukhatme, V.P., Griffioen, A.W., Mayo, K.H. Enhanced tumor growth inhibition by anginex in combination with chemotherapy and other anti-angiogenenic therapy. Proceedings of the AACR, 2002, abstract.

Dings, R.P.M., Yokoyama, Y., van der Schaft, D.W.J., Ramakrishnan, S., Mayo, K.H., Griffioen, A.W. Anginex, a designed angiogenesis inhibitor, that potently inhibits tumor growth in vivo. Proceedings of the AACR, 2001, abstract.

van der Schaft, D.W.J., Mayo, K.H., Barendsz-Janson, A.F., Dings, R.P.M., StruijkerBoudier, H., Wagstaff, J., Hillen, H.F.P., Griffioen, A.W. Bpep-25, a novel angiogenesis inhibitor for cancer treatment. J. Pathol. 193(S): A47-A47, 2001, abstract.

Ter Steege, J.C., van der Schaft, D.W.J., Reincke, C.M., Wagstaff, J., Dings, R.P.M., Mayo, K.H., Griffioen, A.W. Anginex, a newly designed peptide that inhibits angiogenesis. Clin. Cancer Res. 7(11): 13-13, 2001, abstract. 


\section{References}

1. Breier, G, Damert, A., Plate, K.H. \& Risau, W. Angiogenesis in embryos and ischemic diseases. Thromb Haemost 78, 678-683 (1997).

2. Hamby, J.M. \& Showalter, H.D. Small molecule inhibitors of tumor-promoted angiogenesis, including protein tyrosine kinase inhibitors. Pharmacol Ther 82, 169-193 (1999).

3. Virchow, R. Die Krankhaften Geschwulste. (Hirschwald, Berlin; 1863).

4. Ide, A., Baker, N. \& Warren, S. Vascularization of the Brown-Pearce rabbit epithelioma transplant as seen in the transplant ear chamber. Am. J. Roentgenol. 42, 891-899 (1939).

5. Folkman, J. Tumor angiogenesis: therapeutic implications. N Engl J Med 285, 1182-1186 (1971).

6. Folkman, J. Anti-angiogenesis: new concept for therapy of solid tumors. Ann Surg 175, 409416 (1972).

7. Shing, Y. et al. Heparin affinity: purification of a tumor-derived capillary endothelial cell growth factor. Science 223, 1296-1299 (1984).

8. O'Reilly, M.S. et al. Angiostatin: a novel angiogenesis inhibitor that mediates the suppression of metastases by a Lewis lung carcinoma. Cell 79, 315-328 (1994).

9. O'Reilly, M.S. et al. Endostatin: an endogenous inhibitor of angiogenesis and tumor growth. Cell 88, 277-285 (1997).

10. Folkman, J. Angiogenesis in cancer, vascular, rheumatoid and other disease. Nat Med1, 27-31 (1995).

11. Folkman, J. Looking for a good endothial address. Cancer Cell 1, 113-115 (2002).

12. Shweiki, D., Itin, A., Soffer, D. \& Keshet, E. Vascular endothelial growth factor induced by hypoxia may mediate hypoxia-initiated angiogenesis. Nature 359, 843-845 (1992).

13. Ruoslahti, E. How cancer spreads. Sci Am 275, $72-77$ (1996).

14. Kohn, E.C. \& Liotta, L.A. Molecular insights into cancer invasion: strategies for prevention and intervention. Cancer Res 55, 1856-1862 (1995).

15. Alvarez, A.A., Krigman, H.R., Whitaker, R.S., Dodge, R.K. \& Rodriguez, G.C. The prognostic significance of angiogenesis in epithelial ovarian carcinoma. Clin Cancer Res 5, 587-591 (1999).

16. Madri, J.A. Extracellular matrix modulation of vascular cell behaviour. Transp/ Immuno/ 5, 179183 (1997).

17. Leenders, W.P., Kusters, B. \& de Waal, R.M. Vessel co-option: how tumors obtain blood supply in the absence of sprouting angiogenesis. Endothelium 9, 83-87 (2002).

18. Dome, B., Paku, S., Somlai, B. \& Timar, J. Vascularization of cutaneous melanoma involves vessel co-option and has clinical significance. J Pathol 197, 355-362 (2002).

19. Folberg, R., Hendrix, M.J. \& Maniotis, A.J. Vasculogenic mimicry and tumor angiogenesis. Am $J$ Pathol 156, 361-381 (2000).

20. McDonald, D.M. \& Foss, A.J. Endothelial cells of tumor vessels: abnormal but not absent. Cancer Metastasis Rev 19, 109-120 (2000).

21. Ferrara, N. \& Davis-Smyth, T. The biology of vascular endothelial growth factor. Endocr Rev 18, 4-25 (1997).

22. Grosskreutz, C.L. et al. Vascular endothelial growth factor-induced migration of vascular smooth muscle cells in vitro. Microvasc Res 58, 128-136 (1999).

23. Carmeliet, P. \& Collen, D. Role of vascular endothelial growth factor and vascular endothelial growth factor receptors in vascular development. Curr Top Microbiol Immunol 237, 133-158 (1999).

24. Quinn, T.P., Peters, K.G. De Vries, C., Ferrara, N. \& Williams, L.T. Fetal liver kinase 1 is a receptor for vascular endothelial growth factor and is selectively expressed in vascular endothelium. Proc Natl Acad Sci U S A 90, 7533-7537 (1993).

25. Szabo, S. et al. Accelerated healing of duodenal ulcers by oral administration of a mutein of basic fibroblast growth factor in rats. Gastroenterology 106, 1106-1111 (1994). 
26. Fong, G.H., Rossant, J., Gertsenstein, M. \& Breitman, M.L. Role of the Flt-1 receptor tyrosine kinase in regulating the assembly of vascular endothelium. Nature 376, 66-70 (1995).

27. Shalaby, F. et al. Failure of blood-island formation and vasculogenesis in Flk-1-deficient mice. Nature 376, 62-66 (1995).

28. Rak, J. \& Kerbel, R.S. bFGF and tumor angiogenesis-back in the limelight? Nat Med 3, 10831084 (1997).

29. Kong, H.L. \& Crystal, R.G. Gene therapy strategies for tumor antiangiogenesis. J Nat/ Cancer Inst 90, 273-286 (1998).

30. Derbyshire, E.J. \& Thorpe, P.E. in Tumor Angiogenesis. (eds. B. R.L. \& C. E.) 343-356 (Oxford University Press, Oxford; 1997).

31. Hansen-Algensteadt, N. et al. Tumor oxygenation in hormone-dependent tumors during vascular endothelial growth factor receptor-2 blockade, hormone ablation, and chemotherapy. Cancer Res 60, 4556-4560 (2000).

32. Wang, Y. \& Becker, D. Antisense targeting of basic fibroblast growth factor and fibroblast growth factor receptor-1 in human melanomas blocks intratumoral angiogenesis and tumor growth. Nat Med 3, 887-893 (1997).

33. Lin, P. et al. Inhibition of tumor growth by targeting tumor endothelium using a soluble vascular endothelial growth factor receptor. Cell Growth Differ 9, 49-58 (1998).

34. Fong, T.A. et al. SU5416 is a potent and selective inhibitor of the vascular endothelial growth factor receptor (Flk-1/KDR) that inhibits tyrosine kinase catalysis, tumor vascularization, and growth of multiple tumor types. Cancer Res 59, 99-106 (1999).

35. Ke, L.D. et al. A novel approach to glioma gene therapy: down-regulation of the vascular endothelial growth factor in glioma cells using ribozymes. Int J Oncol 12, 1391-1396 (1998).

36. Griffioen, A.W. et al. Anginex, a designed peptide that inhibits angiogenesis. Biochem J 354, 233-242 (2001).

37. Cao, Y. et al. Kringle domains of human angiostatin. Characterization of the anti-proliferative activity on endothelial cells. J Biol Chem 271, 29461-29467 (1996).

38. Tarui, T., Miles, L.A. \& Takada, Y. Specific interaction of angiostatin with integrin alpha(v)beta(3) in endothelial cells. J Biol Chem 276, $39562-39568$ (2001).

39. Moser, T.L. et al. Angiostatin binds ATP synthase on the surface of human endothelial cells. Proc Natl Acad Sci U S A 96, 2811-2816 (1999).

40. Cao, Y. et al. Kringle 5 of plasminogen is a novel inhibitor of endothelial cell growth. J Biol Chem 272, 22924-22928 (1997).

41. Abad, M.C. et al. The X-ray crystallographic structure of the angiogenesis inhibitor angiostatin. J Mol Biol 318, 1009-1017 (2002).

42. Oh, S.P. et al. Isolation and sequencing of cDNAs for proteins with multiple domains of GlyXaa-Yaa repeats identify a distinct family of collagenous proteins. Proc Natl Acad Sci U S A 10, 4229-4233 (1994).

43. Ramchandran, R. et al. Antiangiogenic activity of restin, NC10 domain of human collagen XV: comparison to endostatin. Biochem Biophys Res Commun 255, 735-739 (1999).

44. Wickstrom, S.A., Alitalo, K. \& Keski-Oja, J. Endostatin associates with integrin alpha5beta1 and caveolin-1, and activates Src via a tyrosyl phosphatase-dependent pathway in human endothelial cells. Cancer Res 62, 5580-5589 (2002).

45. Rehn, M. et al. Interaction of endostatin with integrins implicated in angiogenesis. Proc Natl Acad Sci U S A 98, 1024-1029 (2001).

46. Jiang, L., Jha, V., Dhanabal, M., Sukhatme, V.P. \& Alper, S. Intracellular $\mathrm{Ca}(2+)$ signaling in endothelial cells by the angiogenesis inhibitors endostatin and angiostatin. Am J Physiol 280, C1140-1150 (2001).

47. Hohenester, E., Sasaki, T., Olsen, B.R. \& Timpl, R. Crystal structure of the angiogenesis inhibitor endostatin at $1.5 \mathrm{~A}$ resolution. EMBO J 17, 1656-1664 (1998).

48. Graves, B.J. et al. Insight into E-selectin/ligand interaction from the crystal structure and mutagenesis of the lec/EGF domains. Nature 367, 532-538 (1994). 
49. Dixelius, J. et al. Endostatin-induced tyrosine kinase signaling through the Shb adaptor protein regulates endothelial cell apoptosis. Blood 95, 3403-3411 (2000).

50. Baron, M. et al. 1H NMR assignment and secondary structure of the cell adhesion type III module of fibronectin. Biochemistry 31, 2068-2073 (1992).

51. Tan, K. et al. Crystal structure of the TSP-1 type 1 repeats: a novel layered fold and its biological implication. J Cell Biol 159, 373-382 (2002).

52. Beamer, L.J., Carroll, S.F. \& Eisenberg, D. Crystal structure of human BPI and two bound phospholipids at 2.4 angstrom resolution. Science 276, 1861-1864 (1997).

53. Timpl, R., Wiedemann, H., van Delden, V., Furthmayr, H. \& Kuhn, K. A network model for the organization of type IV collagen molecules in basement membranes. Eur J Biochem 120, 203211 (1981).

54. Maeshima, Y. et al. Distinct antitumor properties of a type IV collagen domain derived from basement membrane. J Biol Chem 275, 21340-21348 (2000).

55. Han, J. et al. A cell binding domain from the alpha3 chain of type IV collagen inhibits proliferation of melanoma cells. J Biol Chem 272, 20395-20401 (1997).

56. Maeshima, Y., Colorado, P.C. \& Kalluri, R. Two RGD-independent alpha vbeta 3 integrin binding sites on tumstatin regulate distinct anti-tumor properties. J Biol Chem 275, 23745-23750 (2000).

57. Maeshima, Y. et al. Identification of the anti-angiogenic site within vascular basement membranederived tumstatin. J Biol Chem 276, 15240-15248 (2001).

58. Brooks, P.C., Silletti, S., von Schalscha, T.L., Friedlander, M. \& Cheresh, D.A. Disruption of angiogenesis by PEX, a noncatalytic metalloproteinase fragment with integrin binding activity. Cell 92, 391-400 (1998).

59. Hughes, S. Bayer drug casts shadow over MMP inhibitors in cancer. SCRIP 20, 2477 (1999).

60. Cha, H., Kopetzki, E., Huber, R., Lanzendorfer, M. \& Brandstetter, H. Structural basis of the adaptive molecular recognition by MMP9. J Mol Biol 320, 1065-1079 (2002).

61. Morla, A., Zhang, Z. \& Ruoslahti, E. Superfibronectin is a functionally distinct form of fibronectin. nature 367, 193-196 (1994).

62. Morla, A. \& Ruoslahti, E. A fibronectin self-assembly site involved in fibronectin matrix assembly: reconstruction in a synthetic peptide. J Cell Biol 118, 421-429 (1992).

63. Yi, M. \& Ruoslahti, E. A fibronectin fragment inhibits tumor growth, angiogenesis, and metastasis. Proc Natl Acad Sci U S A 98, 620-624 (2001).

64. Brooks, P.C., Clark, R.A. \& Cheresh, D.A. Requirement of vascular integrin alpha $v$ beta 3 for angiogenesis. Science 264, 569-571 (1994).

65. Leahy, D.J., Hendrickson, W.A., Aukhil, I. \& Erickson, H.P. Structure of a fibronectin type III domain from tenascin phased by MAD analysis of the selenomethionyl protein. Science 258, 987-991 (1992).

66. Kim, J. et al. Antitumor Activity of the 16-kDa Prolactin Fragment in Prostate Cancer. Cancer Res 63, 386-393 (2003).

67. Ferrara, N., Clapp, C. \& Weiner, R. The $16 \mathrm{~K}$ fragment of prolactin specifically inhibits basal or fibroblast growth factor stimulated growth of capillary endothelial cells. Endocrinology 129, 896-900 (1991).

68. Corbacho, A.M., Martinez De La Escalera, G \& Clapp, C. Roles of prolactin and related members of the prolactin/growth hormone/placental lactogen family in angiogenesis. $J$ Endocrinol 173, 219-238 (2002).

69. Nelson, J. et al. Murine epidermal growth factor peptide (33-42) binds to a YIGSR-specific laminin receptor on both tumor and endothelial cells. J Biol Chem 271, 26179-26186 (1996).

70. Struman, I. et al. Opposing actions of intact and $\mathrm{N}$-terminal fragments of the human prolactin/ growth hormone family members on angiogenesis: an efficient mechanism for the regulation of angiogenesis. Proc Natl Acad Sci U S A 96, 1246-1251 (1999).

71. Yao, L., Pike, S.E. \& Tosato, G. Laminin binding to the calreticulin fragment vasostatin regulates endothelial cell function. J Leukoc Biol 71, 47-53 (2002). 
72. Malinda, K.M. et al. Identification of laminin alpha1 and beta1 chain peptides active for endothelial cell adhesion, tube formation, and aortic sprouting. Faseb J 13, 53-62 (1999).

73. Grant, D.S. et al. Two different laminin domains mediate the differentiation of human endothelial cells into capillary-like structures in vitro. Cell 58, 933-943 (1989).

74. Sasaki, T. \& Timpl, R. Domain IVa of laminin alpha5 chain is cell-adhesive and binds beta1 and alphaVbeta3 integrins through Arg-Gly-Asp. FEBS Lett 509, 181-185 (2001).

75. Gebarowska, D. et al. Synthetic peptides interacting with the 67-kd laminin receptor can reduce retinal ischemia and inhibit hypoxia-induced retinal neovascularization. Am J Pathol 160, 307313 (2002).

76. Hohenester, E., Maurer, P. \& Timpl, R. Crystal structure of a pair of follistatin-like and EF-hand calcium- binding domains in BM-40. Embo J 16, 3778-3786 (1997).

77. Mayo, K.H., llyina, E. \& Park, H. A recipe for designing water-soluble, beta-sheet-forming peptides. Protein Sci 5, 13001-11315 (1996).

78. Ilyina, E., Roongta, V. \& Mayo, K.H. NMR structure of a de novo designed, peptide 33 mer with two distinct, compact beta-sheet folds. Biochemistry 36, 5245-5250 (1997).

79. Jones, E.Y., Stuart, D.I. \& Walker, N.P. Structure of tumour necrosis factor. Nature 338, 225-228 (1989).

80. Eck, M.J., Ultsch, M., Rinderknecht, E., de Vos, A.M. \& Sprang, S.R. The structure of human lymphotoxin (tumor necrosis factor-beta) at 1.9-A resolution. J Biol Chem 267, 2119-2122 (1992).

81. Daopin, S., Piez, K.A., Ogawa, Y. \& Davies, D.R. Crystal structure of transforming growth factor-beta 2: an unusual fold for the superfamily. Science 257, 369-373 (1992).

82. van der Schaft, D.W., Toebes, E.A., Haseman, J.R., Mayo, K.H. \& Griffioen, A.W. Bactericidal/ permeability-increasing protein (BPI) inhibits angiogenesis via induction of apoptosis in vascular endothelial cells. Blood 96, 176-181 (2000).

83. Hull, C., McLean, G., Wong, F., Duriez, P.J. \& Karsan, A. Lipopolysaccharide signals an endothelial apoptosis pathway through TNF receptor-associated factor 6-mediated activation of c-Jun NH2-terminal kinase. J Immunol. 169, 2611-2618 (2002).

84. Guida, E. \& Stewart, A. Influence of hypoxia and glucose deprivation on tumour necrosis factoralpha and granulocyte-macrophage colony-stimulating factor expression in human cultured monocytes. Cell Physiol Biochem 8, 75-88 (1998).

85. Bornstein, P., Armstrong, L.C., Hankenson, K.D., Kyriakides, T.R. \& Yang, Z. Thrombospondin 2, a matricellular protein with diverse functions. Matrix Biol 19, 557-568 (2000).

86. Kyriakides, T.R. et al. Mice that lack thrombospondin 2 display connective tissue abnormalities that are associated with disordered collagen fibrillogenesis, an increased vascular density, and a bleeding diathesis. J Cell Biol 140, 419-430 (1998).

87. Armstrong, L.C. et al. Thrombospondin 2 Inhibits Microvascular Endothelial Cell Proliferation by a Caspase-independent Mechanism. Mol Biol Cell 13, 1893-1905 (2002).

88. Ratel, D., Nasser, V., Dupre, I., Benabid, A.L. \& Berger, F. Antibodies to endostatin in a multifocal glioblastoma patient. Lancet 356, 1656-1657 (2000).

89. Kuenen, B.C. et al. Dose-finding and pharmacokinetic study of cisplatin, gemcitabine, and SU5416 in patients with solid tumors. J Clin Oncol 20, 1657-1667 (2002).

90. Marx, GM. et al. Unexpected serious toxicity with chemotherapy and antiangiogenic combinations: time to take stock! J Clin Oncol 20, 1446-1448 (2002).

91. Fogarty, M. Learning from Angiogenesis Trial Failures. The Scientist 16, 33-35 (2002).

92. Hansen-Algensteadt, N., Fukumura, D., Stoll, B., Hicklin, D. \& Jain, R.K. Second wave of angiogenesis during KDR/flk-1 antibody therapy [abstract]. Proc Am Assoc Cancer Res 40, 620 (1999).

93. Cao, Y., Cao, R. \& Brakenhielm, E. Antiangiogenic mechanisms of diet-derived polyphenols. $J$ Nutr Biochem 13, 380-390 (2002).

94. Mayo, K.H., Haseman, J.R., Ilyina, E. \& Gray, B. Designed beta-sheet-forming peptide 33mers with potent human bactericidal/permeability increasing protein-like bactericidal and endotoxin neutralizing activities. Biochem Biophys Acta 1425, 81-92 (1998). 
95. Mayo, K.H., van der Schaft, D.W. \& Griffioen, A.W. Designed beta-sheet peptides that inhibit proliferation and induce apoptosis in endothelial cells. Angiogenesis 4, 45-51 (2001).

96. Griffioen, A.W. \& Molema, G. Angiogenesis: potentials for pharmacologic intervention in the treatment of cancer, cardiovascular diseases, and chronic inflammation. Pharmacol Rev 52, 237-268 (2000).

97. Folkman, J. \& Klagsbrun, M. Angiogenic factors. Science 235, 442-447 (1987).

98. Hanahan, D. \& Folkman, J. Patterns and emerging mechanisms of the angiogenic switch during tumorigenesis. Cell 86, 353-364 (1996).

99. Williams, S.G, Buscarini, M. \& Stein, J.P. Molecular markers for diagnosis, staging, and prognosis of bladder cancer. Oncology (Huntingt) 15, 1469-1470, 1473-1464, 1476; discussion 14761484 (2001).

100. Weidner, N. Angiogenesis as a predictor of clinical outcome in cancer patients. Hum Patho/31, 403-405 (2000).

101. Rastinejad, F., Polverini, P.J. \& Bouck, N.P. Regulation of the activity of a new inhibitor of angiogenesis by a cancer suppressor gene. Cell 56, 345-355 (1989).

102. Kusaka, M. et al. Potent anti-angiogenic action of AGM-1470: comparison to the fumagillin parent. Biochem Biophys Res Commun 174, 1070-1076 (1991).

103. Gupta, S.K. \& Singh, J.P. Inhibition of endothelial cell proliferation by platelet factor-4 involves a unique action on S phase progression. J Cell Biol 127, 1121-1127 (1994).

104. Onn, A., Tseng, J.E. \& Herbst, R.S. Thalidomide, cyclooxygenase-2, and angiogenesis: potential for therapy. Clin Cancer Res 7, 3311-3313 (2001).

105. Sills, A.K., Jr. et al. Squalamine inhibits angiogenesis and solid tumor growth in vivo and perturbs embryonic vasculature. Cancer Res 58, 2784-2792 (1998).

106. Kerbel, R.S. Tumor angiogenesis: past, present and the near future. Carcinogenesis 21, 505515 (2000).

107. Kisker, O. et al. Continuous administration of endostatin by intraperitoneally implanted osmotic pump improves the efficacy and potency of therapy in a mouse xenograft tumor model. Cancer Res 61, 7669-7674 (2001).

108. Majumdar, M.K., Wang, E. \& Morris, E.A. BMP-2 and BMP-9 promotes chondrogenic differentiation of human multipotential mesenchymal cells and overcomes the inhibitory effect of IL-1. J Cell Physiol 189, 275-284 (2001).

109. Duncan, R.J., Weston, P.D. \& Wrigglesworth, R. A new reagent which may be used to introduce sulfhydryl groups into proteins, and its use in the preparation of conjugates for immunoassay. Anal Biochem 132, 68-73 (1983).

110. Klotz, I.M. \& Heiney, R.E. Introduction of sulfhydryl groups into proteins using acetylmercapto succinic anhydride. Arch. Biochem. Biophys 96, 605-612 (1962).

111. Elman, G.L. Tissue sulfhydryl groups. Arch. Biochem. Biophys 82, 70-77 (1959).

112. Wild, R., Ramakrishnan, S., Sedgewick, J. \& Griffioen, A.W. Quantitative assessment of angiogenesis and tumor vessel architecture by computer-assisted digital image analysis: effects of VEGF-toxin conjugate on tumor microvessel density. Microvasc Res 59, 368-376 (2000).

113. Shalinsky, D.R. et al. Broad antitumor and antiangiogenic activities of AG3340, a potent and selective MMP inhibitor undergoing advanced oncology clinical trials. Ann N Y Acad Sci 878, 236-270 (1999).

114. Takka, S., Ocak, O.H. \& Acarturk, F. Formulation and investigation of nicardipine $\mathrm{HCl}$-alginate gel beads with factorial design-based studies. Eur J Pharm Sci 6, 241-246 (1998).

115. Read, T.A. et al. Local endostatin treatment of gliomas administered by microencapsulated producer cells. Nat Biotechnol 19, 29-34 (2001).

116. Arica, B., Calis, S., Kas, H., Sargon, M. \& Hincal, A. 5-Fluorouracil encapsulated alginate beads for the treatment of breast cancer. Int J Pharm 242, 267 (2002).

117. Mehvar, R., Robinson, M.A. \& Reynolds, J.M. Molecular weight dependent tissue accumulation of dextrans: in vivo studies in rats. J Pharm Sci 83, 1495-1499 (1994). 
118. Yamaoka, T., Tabata, Y. \& Ikada, Y. Distribution and tissue uptake of poly(ethylene glycol) with different molecular weights after intravenous administration to mice. J Pharm Sci 83, 601-606 (1994).

119. Dosi, F., Brusa, P., Crosasso, P., Arpicco, S. \& Cattel, L. Preparation, characterization and properties in vitro and in vivo of a paclitaxel-albumin conjugate. J. Controlled Release 47, 293304 (1997).

120. Bruns, C.J. et al. Epidermal growth factor receptor blockade with C225 plus gemcitabine results in regression of human pancreatic carcinoma growing orthotopically in nude mice by antiangiogenic mechanisms. Clin Cancer Res 6, 1936-1948 (2000).

121. Molema, G. \& Griffioen, A.W. Rocking the foundations of solid tumor growth by attacking the tumor's blood supply. Immunol Today 19, 392-394 (1998).

122. Friedlander, M. et al. Definition of two angiogenic pathways by distinct alpha $v$ integrins. Science 270, 1500-1502 (1995).

123. Gupta, S.K., Hassel, T. \& Singh, J.P. A potent inhibitor of endothelial cell proliferation is generated by proteolytic cleavage of the chemokine platelet factor 4. Proc Natl Acad Sci U S A 92, 77997803 (1995).

124. Good, D.J. et al. A tumor suppressor-dependent inhibitor of angiogenesis is immunologically and functionally indistinguishable from a fragment of thrombospondin. Proc Natl Acad Sci US A 87, 6624-6628 (1990).

125. Luster, A.D., Greenberg, S.M. \& Leder, P. The IP-10 chemokine binds to a specific cell surface heparan sulfate site shared with platelet factor 4 and inhibits endothelial cell proliferation. $J$ Exp Med 182, 219-231 (1995).

126. Pike, S.E. et al. Vasostatin, a calreticulin fragment, inhibits angiogenesis and suppresses tumor growth. J Exp Med 188, 2349-2356 (1998).

127. Harder, R. et al. Dissection of murine lymphocyte-endothelial cell interaction mechanisms by SV-40-transformed mouse endothelial cell lines: novel mechanisms mediating basal binding, and alpha 4-integrin-dependent cytokine- induced adhesion. Exp Cell Res 197, 259-267 (1991).

128. Griffioen, A.W., Damen, C.A., Martinotti, S., Blijham, G.H. \& Groenewegen, G. Endothelial intercellular adhesion molecule-1 expression is suppressed in human malignancies: the role of angiogenic factors. Cancer Res 56, 1111-1117 (1996).

129. Nicholson, D.W. et al. Identification and inhibition of the ICE/CED-3 protease necessary for mammalian apoptosis. Nature 376, 37-43 (1995).

130. Fujita, E. et al. Enhancement of CPP32-like activity in the TNF-treated U937 cells by the proteasome inhibitors. Biochem Biophys Res Commun 224, 74-79 (1996).

131. Slee, E.A. et al. Benzyloxycarbonyl-Val-Ala-Asp $(\mathrm{OMe})$ fluoromethylketone (Z-VAD.FMK) inhibits apoptosis by blocking the processing of CPP32. Biochem J 315, 21-24 (1996).

132. van der Schaft, D.W., Wagstaff, J., Mayo, K.H. \& Griffioen, A.W. The antiangiogenic properties of bactericidal/permeability-increasing protein (BPI). Ann Med34, 19-27 (2002).

133. Yamaoka, M. et al. Inhibition of tumor growth and metastasis of rodent tumors by the angiogenesis inhibitor O-(chloroacetyl-carbamoyl)fumagillol (TNP-470; AGM-1470). Cancer Res 53, 42624267 (1993).

134. Yokoyama, Y., Dhanabal, M., Griffioen, A.W., Sukhatme, V.P. \& Ramakrishnan, S. Synergy between angiostatin and endostatin: inhibition of ovarian cancer growth. Cancer Res 60, 21902196 (2000).

135. Hillen, H.F., Hak, L.E., Joosten-Achjanie, S.R. \& Arends, J.W. Microvessel density in unknown primary tumors. Int J Cancer 74, 81-85 (1997).

136. van Dijke, C.F. et al. Mammary carcinoma model: correlation of macromolecular contrastenhanced MR imaging characterizations of tumor microvasculature and histologic capillary density. Radiology 198, 813-818 (1996).

137. Salvesen, G.S. \& Dixit, V.M. Caspases: intracellular signaling by proteolysis. Cell $91,443-446$ (1997). 
138. Yamaoka, M., Yamamoto, T., Ikeyama, S., Sudo, K. \& Fujita, T. Angiogenesis inhibitor TNP-470 (AGM-1470) potently inhibits the tumor growth of hormone-independent human breast and prostate carcinoma cell lines. Cancer Res 53, 5233-5236 (1993).

139. Jain, R.K., Schlenger, K., Hockel, M. \& Yuan, F. Quantitative angiogenesis assays: progress and problems. Nat Med 3, 1203-1208 (1997).

140. Bouma-ter Steege, J.C., Mayo, K.H. \& Griffioen, A.W. Angiostatic proteins and peptides. Crit Rev Eukaryot Gene Expr 11, 319-334 (2001).

141. Griffioen, A.W. et al. CD44 is involved in tumor angiogenesis; an activation antigen on human endothelial cells. Blood 90, 1150-1159 (1997).

142. Karumanchi, S.A. et al. Cell surface glypicans are low-affinity endostatin receptors. Mol Cell 7, 811-822 (2001).

143. Jimenez, B. et al. Signals leading to apoptosis-dependent inhibition of neovascularization by thrombospondin-1. Nat Med 6, 41-48 (2000).

144. Hanahan, D. \& Weinberg, R.A. The hallmarks of cancer. Cell 100, 57-70 (2000).

145. Gorski, D.H. et al. Potentiation of the antitumor effect of ionizing radiation by brief concomitant exposures to angiostatin. Cancer Res 58, 5686-5689 (1998).

146. Mauceri, H.J. et al. Combined effects of angiostatin and ionizing radiation in antitumour therapy. Nature 394, 287-291 (1998).

147. Wilczynska, U., Kucharska, A., Szary, J. \& Szala, S. Combined delivery of an antiangiogenic protein (angiostatin) and an immunomodulatory gene (interleukin-12) in the treatment of murine cancer. Acta Biochim Pol 48, 1077-1084 (2001).

148. Teicher, B.A., Sotomayor, E.A. \& Huang, Z.D. Antiangiogenic agents potentiate cytotoxic cancer therapies against primary and metastatic disease. Cancer Res 52, 6702-6704 (1992).

149. Dhanabal, M. et al. Endostatin: yeast production, mutants, and antitumor effect in renal cell carcinoma. Cancer Res 59, 189-197 (1999).

150. Mohanraj, D., Olson, T. \& Ramakrishnan, S. Expression of biologically active human vascular endothelial growth factor in yeast. Growth Factors 12, 17-27 (1995).

151. Harries, M. \& Kaye, S.B. Recent advances in the treatment of epithelial ovarian cancer. Expert Opin Investig Drugs 10, 1715-1724 (2001).

152. Tattersall, M.H.N. Ovarian cancer chemotherapy: carboplatin as standard. The Lancet 360 , 500-501 (2002).

153. ICON Paclitaxel plus carboplatin versus standard chemotherapy with either single-agent carboplatin or cyclophosphamide, doxorubicin, and cisplatin in women with ovarian cancer: the ICON3 randomised trial. The Lancet 360, 505-515 (2002).

154. Dings, R.P.M. et al. Anti-tumor activity of the novel angiogenesis inhibitor anginex. Can Lett 194, 55-66 (2003).

155. Dings, R.P., Yokoyama, Y., Ramakrishnan, S., Griffioen, A.W. \& Mayo, K.H. The designed angiostatic peptide anginex synergistically improves chemotherapy and antiangiogenesis therapy with angiostatin. Cancer Res 63, 382-385 (2003).

156. van der Schaft, D.W. et al. The designer anti-angiogenic peptide anginex targets tumor endothelial cells and inhibits tumor growth in animal models. Faseb J 16, 1991-1993 (2002).

157. Adler, A.J., Greenfield, N.J. \& Fasman, G.D. Circular dichroism and optical rotary dispersion of proteins and polypeptides. Methods in Enzymology 27, 675-735 (1973).

158. Greenfield, N. \& Fasman, G.D. Computed circular dichroism spectra for the evaluation of protein conformation. Biochemistry 8, 4108-4116 (1969).

159. Bax, A. \& Davis, D.G. MLEV-17-based two-dimensional homonuclear magnetization transfer spectroscopy. J. Magn. Reson. 65, 355-360 (1985).

160. Wider, G., Macura, S., Anil-Kumar, Ernst, R.R. \& Wüthrich, K. Homonuclear two-dimensional 'H NMR proteins. J. Magn. Reson. 56, 207-234 (1985).

161. States, D.J., Haberkorn, R.A. \& Ruben, D.J. A two-dimensional nuclear Overhauser experiment with pure absorbtion phase in four quadrants. J. Magn. Reson. 48, 286-292 (1982). 
162. Bodenhausen, G, Vold, R.L. \& Vold, R.R. Multiple quantum spin echo spectroscopy. J. Magn. Reson. 37, 93-99 (1980).

163. Delaglio, F. et al. NMRPipe: a multidimensional spectral processing system based on UNIX pipes. J Biomol NMR 6, 277-293 (1995).

164. Brunger, A.T. X-plor Manual. (Yale University Press, New Haven; 1992).

165. Relou, I.A., Damen, C.A., van der Schaft, D.W., Groenewegen, G. \& Griffioen, A.W. Effect of culture conditions on endothelial cell growth and responsiveness. Tissue Cell 30, 525-530 (1998).

166. Darzynkiewicz, Z. et al. Features of apoptotic cells measured by flow cytometry. Cytometry 13, 795-808 (1992).

167. Barendsz-Janson, A.F., Griffioen, A.W., Muller, A.D., van Dam-Mieras, M.C. \& Hillen, H.F. In vitro tumor angiogenesis assays: plasminogen lysine binding site 1 inhibits in vitro tumor-induced angiogenesis. J Vasc Res 35, 109-114 (1998).

168. Johnson, W.C.J. Protein secondary structure and circular dichroism: a practical guide. Proteins 7, 205-214 (1990).

169. Waterhous, D.V. \& Johnson, W.C., Jr. Importance of environment in determining secondary structure in proteins. Biochemistry 33, 2121-2128 (1994).

170. Hanzawa, $\mathrm{H}$. et al. $1 \mathrm{H}$ nuclear magnetic resonance study of the solution conformation of an antibacterial protein, sapecin. FEBS Lett 269, 413-420 (1990).

171. Selsted, M.E. \& Harwig, S.S. Determination of the disulfide array in the human defensin HNP-2. A covalently cyclized peptide. J Biol Chem 264, 4003-4007 (1989).

172. Mayo, K.H. et al. NMR solution structure of the 32-kDa platelet factor 4 ELR-motif N-terminal chimera: a symmetric tetramer. Biochemistry 34, 11399-11409 (1995).

173. Baeyens, K.J., De Bondt, H.L., Raeymaekers, A., Fiers, W. \& De Ranter, C.J. The structure of mouse tumour-necrosis factor at 1.4 A resolution: towards modulation of its selectivity and trimerization. Acta Crystallogr D Biol Crystallogr 55, $772-778$ (1999).

174. Boehm, T., Folkman, J., Browder, T. \& O'Reilly, M.S. Antiangiogenic therapy of experimental cancer does not induce acquired drug resistance. Nature 390, 404-407 (1997).

175. Tolsma, S.S. et al. Peptides derived from two separate domains of the matrix protein thrombospondin-1 have anti-angiogenic activity. J Cell Biol 122, 497-511 (1993).

176. Dings, R.P.M. et al. B-sheet is the bioactive conformation of the anti-angiogenic anginex peptide. Biochem J 373, 1-8 (2003).

177. Bekele, H. et al. Improved Synthesis of the Boc and Fmoc Derivatives of 4-(2'-Aminoethyl)- 6dibenzofuranpropionic Acid: An Unnatural Amino Acid That Nucleates beta-Sheet Folding. $J$ Org Chem 62, 2259-2262 (1997).

178. Carpino, L.A. 1-hydroxy-7-azabenzotriazole. An efficient peptide coupling additive. J. Am. Chem. Soc. 115, 4397-4398 (1993).

179. King, D.S., Fields, C.G \& Fields, GB. A cleavage method which minimizes side reactions following Fmoc solid phase peptide synthesis. Int J Pept Protein Res 36, 255-266. (1990).

180. Piotto, M., Saudek, V. \& Sklenar, V. Gradient-tailored excitation for single-quantum NMR spectroscopy of aqueous solutions. J Biomol NMR 2, 661-665. (1992).

181. Shaka, A.J., Lee, C.J. \& Pines, A. Iterative Schemes for Bilinear Operators: Applications to Spin Decoupling. J. Magn. Reson. 77, 274-293 (1988).

182. Rucker, S.P. \& Shaka, A.J. Broadband homonuclear cross polarization in 2D NMR using DIPSI2. Mol. Phys. 68, 509-517 (1989).

183. Diaz, H., Tsang, K.Y., D., C., J.R., E. \& J.W., K. Design, synthesis, and partial characterization of water-soluble B-sheets stabilized by a dibenzofuran-based amino acid. J. Am. Chem. Soc. 115, 3790-3791 (1993).

184. Tsang, K.Y., Diaz, H., Graciani, N. \& Kelly, J.W. Hydrophobic cluster formation is necessary for dibezofuran-based amino acids to function as B-sheet nucleators. J. Am. Chem. Soc. 116, 3988-4005 (1994). 
185. Wiesmann, C. et al. Crystal structure at $1.7 \mathrm{~A}$ resolution of VEGF in complex with domain 2 of the Flt-1 receptor. Cell 91, 695-704 (1997).

186. Tan, D.C. et al. A small peptide derived from Flt-1 (VEGFR-1) functions as an angiogenic inhibitor. FEBS Lett 494, 150-156 (2001).

187. Kranenburg, $\mathrm{O}$. et al. Tissue-type plasminogen activator is a multiligand cross-beta structure receptor. Curr Biol 12, 1833-1839 (2002).

188. MacDonald, N.J. et al. Endostatin binds tropomyosin. A potential modulator of the antitumor activity of endostatin. J Biol Chem 276, 25190-25196 (2001).

189. Chen, H., Herndon, M.E. \& Lawler, J. The cell biology of thrombospondin-1. Matrix Biol 19, 597614 (2000).

190. Dawson, D.W. et al. CD36 mediates the In vitro inhibitory effects of thrombospondin-1 on endothelial cells. J Cell Biol 138, 707-717 (1997).

191. Kerbel, R.S. Clinical trials of antiangiogenic drugs: opportunities, problems, and assessment of initial results. J Clin Oncol 19, 45S-51S (2001).

192. Teicher, B.A. et al. Potentiation of cytotoxic cancer therapies by TNP-470 alone and with other anti-angiogenic agents. Int J Cancer 57, 920-925 (1994).

193. Dings, R.P.M., Nesmelova, I., Griffioen, A.W. \& Mayo, K.H. Discovery and development of antiangiogenic peptides: a structural link. Angiogenesis 5, 1-12 (2003).

194. Dawson, D.W. et al. Three distinct D-amino acid substitutions confer potent antiangiogenic activity on an inactive peptide derived from a thrombospondin-1 type 1 repeat. Mol Pharmacol 55, 332-338 (1999).

195. Reiher, F.K. et al. Inhibition of tumor growth by systemic treatment with thrombospondin-1 peptide mimetics. Int J Cancer 98, 682-689 (2002).

196. Maeshima, Y. et al. Extracellular matrix-derived peptide binds to alpha(v)beta(3) integrin and inhibits angiogenesis. J Biol Chem 276, 31959-31968 (2001).

197. Bakre, M.M. et al. Parathyroid hormone-related peptide is a naturally occurring, protein kinase A-dependent angiogenesis inhibitor. Nat Med 8, 995-1003 (2002).

198. Arap, W., Pasqualini, R. \& Ruoslahti, E. Cancer treatment by targeted drug delivery to tumor vasculature in a mouse model. Science 279, 377-380 (1998).

199. Pasqualini, R., Koivunen, E. \& Ruoslahti, E. Alpha v integrins as receptors for tumor targeting by circulating ligands. Nat Biotechnol 15, 542-546 (1997).

200. Sgadari, C., Angiolillo, A.L. \& Tosato, G Inhibition of angiogenesis by interleukin-12 is mediated by the interferon-inducible protein 10. Blood 87, 3877-3882 (1996).

201. Fairbrother, W.J. et al. Novel peptides selected to bind vascular endothelial growth factor target the receptor-binding site. Biochemistry 37, 17754-17764 (1998).

202. Pan, B. et al. Solution structure of a phage-derived peptide antagonist in complex with vascular endothelial growth factor. J Mol Bio/316, 769-787 (2002).

203. Davis-Smyth, T., Chen, H., Park, J., Presta, L.G. \& Ferrara, N. The second immunoglobulin-like domain of the VEGF tyrosine kinase receptor Flt-1 determines ligand binding and may initiate a signal transduction cascade. Embo J 15, 4919-4927 (1996). 

Angiogenesis, the sprouting of capillaries from pre-existing blood vessels, is involved in normal physiological processes of tissue development as well as in the pathogenesis of diseases, such as diabetic retinopathy or retinopathy of prematurity, psoriasis, atherosclerosis, obesity, rheumatoid arthritis, peptic ulcers, fractures and cancer.

Although pre-clinical testing of anti-angiogenic agents shows promise, the need for more and better angiogenesis inhibitors is driven by the absence of any major clinical breakthroughs. The development of new angiogenesis inhibitors, especially synthetic small peptides, is an important approach in improving or even replacing the current anti-angiogenic therapy. Here, it is shown for the first time that angiogenesis inhibitor anginex (33-mer) poses antitumor activity in vivo, in both syngeneic and xenograft mouse models. Tumor growth was inhibited synergistically when anginex was combined with angiostatin. Moreover tumors regressed to an impalpable state when anginex was combined with conventional treatment. Structural activity relationships within anginex resulted in the design and development of a new generation of peptide-mimetics with improved tumor inhibitory activity.

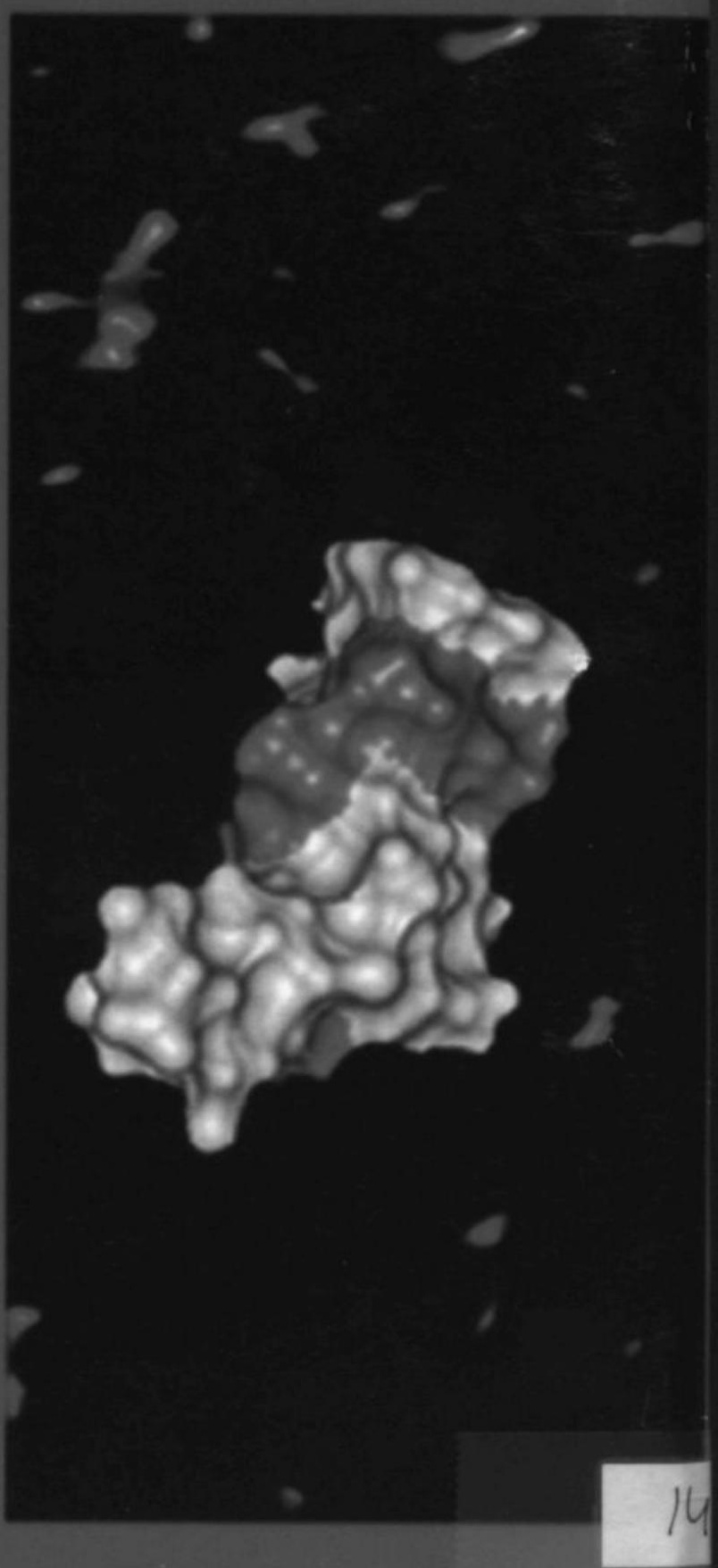

